\title{
The Bidiagonal Singular Value Decomposition and Hamiltonian Mechanics
}

\author{
Percy Deift \\ James Demme ${ }^{1}$ \\ Courant Institute \\ New YorkTNY 10012 \\ Chau Li \\ Mathematics Department \\ The Pennsylvania State University \\ University ParkГPA 16802 \\ Carlos Tomei \\ $\mathrm{PUC}$ \\ Rio de JaneiroTBrazil \\ (visiting Yale University)
}

\begin{abstract}
We consider computing the singular value decomposition of a bidiagonal matrix $B$. This problem arises in the singular value decomposition of a general matrix $\Gamma$ and in the eigenproblem for a symmetric positive definite tridiagonal matrix. We show that if the entries of $B$ are known with high relative accuracyCthe singular values and singular vectors of $B$ will be determined to much higher accuracy than the standard perturbation theory suggests. We also show that the algorithm in [Demmel and Kahan] computes the singular vectors as well as the singular values to this accuracy. We also give a Hamiltonian interpretation of the algorithm and use differential equation methods to prove many of the basic facts. The Hamiltonian approach suggests a way to use flows to predict the accumulation of error in other eigenvalue algorithms as well.
\end{abstract}

(This paper appeared in the SIAM J. Numer. Anal.Tv. 18Гn. 5Гpp. 1463-1516Г1991) 


\section{Introduction}

The singular value decomposition (SVD) of a real general $n$ by $n$ matrix $A$ is the factorization $A=U \Sigma V^{T}$ Twhere $U$ and $V$ are orthogonal $\Gamma=\operatorname{diag}\left\{\sigma_{1}, \ldots, \sigma_{n}\right\}$ Tand $\sigma_{1} \geq \cdots \geq \sigma_{n} \geq 0$. The $\sigma_{i}$ 's are the singular values of $A$ T the columns $v_{i}$ of $V$ the right singular vectors of $A \Gamma$ and the columns $u_{i}$ of $U$ the left singular vectors of $A$.

In this paper we will consider the SVD of a bidiagonal matrix $B$

$$
B=\left[\begin{array}{cccc}
a_{1} & b_{1} & & \\
& \ddots & \ddots & \\
& & \ddots & b_{n-1} \\
& & & a_{n}
\end{array}\right],
$$

where we may assume without loss of generality that the $a_{i}$ and $b_{i}$ are positive. (Recall that this assumption implies that all the $\sigma_{i}$ are positive and distinct [Par80].) This problem arises as the final stage of the SVD of a general matrix $A$ [GK65T GVL83] $\Gamma$ as well as in computing the eigendecomposition of a symmetric positive-definite tridiagonal matrix $T$ [BD88]. Both computations arise frequently in a wide variety of applications. Our goal in this paper is threefold: to show that the SVD of a bidiagonal $B$ can be computed much more accurately than the SVD of a general matrix $A \Gamma$ to explain this with the aid of a Hamiltonian differential equation underlying the SVD algorithm used for $B$ Cand to suggest using similar differential equations to find high accuracy algorithms for other eigenvalue and singular value problems.

How accurately can the SVD of a general matrix $A$ be computed? To answer this question $\Gamma$ we must consider both the effects of uncertainty in the initial data $A$ Tas well as errors introduced by an algorithm (roundoff errors and the effects of the stopping criterion); a good algorithm introduces errors no worse than inherent errors caused by uncertainty in the data. The standard approach is to bound the uncertainty $\delta A$ in the initial data by its two-norm $\|\delta A\|$ : we say that $\delta A$ is an absolute error of scale $\eta$ in $A$ if $\|\delta A\| /\|A\| \leq \eta$. With this definition of $\eta$ we have:

(A) - The singular values $\sigma_{i}$ of $A$ and $\sigma_{i}^{\prime}$ of $A+\delta A$ can differ by at most $\eta\|A\|$ for all $i$ [GVL83]:

$$
\left|\sigma_{i} \Leftrightarrow \sigma_{i}^{\prime}\right| \leq \eta\|A\|
$$

(B) - Let $u_{i}$ and $u_{i}^{\prime}$ be the $i$-th left unit singular vectors of $A$ and $A+\delta A$ respectivelyTand $v_{i}$ and $v_{i}^{\prime}$ be the right unit singular vectors. Let the absolute gap for $\sigma_{i}$ be defined as the distance from $\sigma_{i}$ to the nearest different singular valueTnormalized by $\sigma_{1}$ :

$$
\operatorname{absgap}_{i} \equiv \min _{j \neq i}\left|\sigma_{i} \Leftrightarrow \sigma_{j}\right| / \sigma_{1}
$$

If $\eta<a b \operatorname{sgap}_{i} / 2 \Gamma$ then the sines of the angles between $u_{i}$ and $u_{i}^{\prime}\left(\sin \theta\left(u_{i}, u_{i}^{\prime}\right)\right) \Gamma$ and between $v_{i}$ and $v_{i}^{\prime}\left(\sin \theta\left(v_{i}, v_{i}^{\prime}\right)\right)$ Tare bounded as follows[DK70]:

$$
\max \left(\sin \theta\left(u_{i}, u_{i}^{\prime}\right), \sin \theta\left(v_{i}, v_{i}^{\prime}\right)\right) \leq \frac{\eta}{a b s g a p_{i} \Leftrightarrow \eta}
$$


The reason for limiting $\eta<a b s g a p_{i} / 2$ is that for larger perturbations the bound (1.4) is vacuous. This reflects the fact that larger perturbations by a general matrix can cause $\sigma_{i}$ to merge with another singular value so that the singular vectors fail to be uniquely defined.

Now we consider the errors introduced by the standard algorithm [GK65] for the SVD of a general matrix. We assume a standard model of floating point arithmetic 5 with relative errors of size at most $\varepsilon$ The machine precision $\Gamma$ in each basic operation $\Gamma$ and assume neither overflow nor underflow occur. Under these assumptions it is well known [GVL83] that the error bound of the standard algorithm is equivalent to an uncertainty $\delta A$ in the initial data with absolute error scale $\|\delta A\| /\|A\| \leq p(n) \varepsilon \Gamma$ where $p(n)$ depends on details of the arithmetic and algorithm $\Gamma$ and is a modestly growing function of the dimension $n$ of $A$ (a cubic polynomial in $n$ with modest coefficients). In other wordsT the error introduced by the algorithm is no worse than $p(n) \varepsilon\|A\|=p(n) \varepsilon \sigma_{1}$ in the singular values and no worse than $p(n) \varepsilon / a b$ sgap $_{i}$ in the singular vectors.

In particularTsuppose $A$ has one or more tiny singular values $\sigma_{j}$ Twhere by tiny we mean $\sigma_{j} \ll \sigma_{1}=\|A\|$. The error bound $p(n) \varepsilon \sigma_{1}$ for the singular values implies that while large singular values may be computed with high relative accuracy tiny ones will in general not be $\mathrm{since}$ the error bound may greatly exceed their value. Also if there are two or more tiny singular values Ttheir absolute gaps will necessarily be small compared to $\sigma_{1}$ Tand their singular vectors correspondingly uncertain. Indeed $\Gamma$ these uncertainties are unavoidable as long as one considers general perturbations $\delta A$ bounded only in norm in (A) and (B) above are attainable [Wil65].

Now we return to the case of a bidiagonal matrix $B$. It turns out that the effects of both initial data uncertainties and roundoff errors are significantly smaller than for general matricesTand are controlled by the relative error of $\delta B$

$$
\eta_{r} \equiv(2 n \Leftrightarrow 1) \cdot \max _{i j}\left|\log \frac{B_{i j}+\delta B_{i j}}{B_{i j}}\right|
$$

instead of by its norm $\|\delta B\|$. When $\eta_{r} \ll 1 \Gamma$ this means that the sum of the componentwise relative errors $\sum_{i j}\left|\frac{\delta B_{i j}}{B_{i j}}\right|$ is approximately bounded by $\eta_{r}$. In other words $\Gamma$ the zero entries of $B$ must remain zeroTand we only permit relative perturbations in the remaining entries $\Gamma$ rather than norm bounded perturbations. Using $\eta_{r}$ as measure of uncertainty in $B$ Tone can prove:

$\left(\mathbf{A}^{\prime}\right)$ - Let the $\sigma_{i}$ be the singular values of $B$ and $\sigma_{i}^{\prime}$ be the singular values of $B+\delta B$. Then

$$
e^{-\eta_{r}} \leq \frac{\sigma_{i}^{\prime}}{\sigma_{i}} \leq e^{\eta_{r}}
$$

This bound holds for all $\eta_{r} \geq 0$ Cjust as (1.2) holds for all $\eta \geq 0$. When $\eta_{r} \ll 1 \Gamma$ these upper and lower bounds on $\frac{\sigma_{i}^{\prime}}{\sigma_{i}}$ are approximately $1 \pm \eta_{r}$ Tmeaning that small relative perturbations in the entries of $B$ only cause small relative perturbations in the $\sigma_{i} \Gamma$ independent of their magnitudes. 
$\left(\mathbf{B}^{\prime}\right)$ - For simplicity of notation write $e^{\eta_{r}}=1+\eta_{r}^{\prime}$; when $\eta_{r} \ll 1 \Gamma \eta_{r} \approx \eta_{r}^{\prime}$. Let $u_{i}$ and $v_{i}$ be the singular vectors of $B$ Tand let $u_{i}^{\prime}$ and $v_{i}^{\prime}$ be the singular vectors of $B+\delta B$. Let the relative gap for $\sigma_{i}$ be defined as the relative distance from $\sigma_{i}$ to the nearest different singular value:

$$
\operatorname{relgap}_{i} \equiv \min _{j \neq i}\left|\sigma_{i} \Leftrightarrow \sigma_{j}\right| /\left|\sigma_{i}+\sigma_{j}\right|
$$

If $\eta_{r}^{\prime}<\operatorname{relgap}_{i}$ Tthen the sines of the angles between $u_{i}$ and $u_{i}^{\prime}$ Tand between $v_{i}$ and $v_{i}^{\prime}$ Tare bounded by

$$
\max \left(\sin \theta\left(u_{i}, u_{i}^{\prime}\right), \sin \theta\left(v_{i}, v_{i}^{\prime}\right) \leq \frac{2^{1 / 2} \eta_{r}^{\prime}\left(1+\eta_{r}^{\prime}\right)}{\text { relgap }_{i} \Leftrightarrow \eta_{r}^{\prime}}\right.
$$

Result ( $\mathbf{A}^{\prime}$ ) was proven in [Kah68Г DK88Г BD88]; we include a (new) proof for the convenience of the reader. Result $\left(\mathbf{B}^{\prime}\right)$ is proved here for the first time; it was conjectured in [DK88] with a proof of a weaker result in [BD88].

One can easily see that results $\left(\mathbf{A}^{\prime}\right)$ and $\left(\mathbf{B}^{\prime}\right)$ are always at least about as strong as their counterparts (A) and (B). To show how much stronger they may be $\mathrm{A}$ consider making relative perturbations of size $10^{-10}$ in a 3 by 3 bidiagonal matrix with singular values $\sigma_{1}=1 \Gamma \sigma_{2}=2 \cdot 10^{-20} \Gamma$ and $\sigma_{3}=10^{-20}$. Note that $a b s g a p_{3}=a b s g a p_{2}=10^{-20} \Gamma$ and that relgap $_{3}=$ relgap $_{2}=1 / 3$. Since the norm of this perturbation is about $10^{-10}$ Twe may apply $(\mathbf{A})$ and $(\mathbf{B})$ to get the absolute error bound $10^{-10} \gg \sigma_{3}$ for $\sigma_{3}$ Гand $\Gamma$ since $10^{-10} \gg a b s g a p_{3} \Gamma$ no error bound for the singular vectors at all. Applying $\left(\mathbf{A}^{\prime}\right)$ Twe get a relative error bound of about $5 \cdot 10^{-10}$ in $\sigma_{3}$. Thus Twe have at least 9 decimal digits of accuracy in $\sigma_{3}$ Twhereas (A) predicts changes $10^{20}$ times larger. Applying $\left(\mathbf{B}^{\prime}\right) \Gamma$ we get an error bound of about $2.1 \cdot 10^{-9}$ in the direction of the singular vectors $\Gamma$ whereas $(\mathbf{B})$ provides no error bound at all. The same results hold for $\sigma_{2}$ and its singular vectors.

In summaryГ absolute uncertainties in the entries of a general matrix $A$ yield absolute error bounds on its singular values $\Gamma$ and error bounds depending on the absolute gap for its singular vectors. In contrast Trelative uncertainties in the entries of a bidiagonal matrix $B$ yield relative error bounds on its singular values $\Gamma$ and error bounds depending on the relative gap for its singular vectors.

Given the much greater accuracy to which singular values and singular vectors of bidiagonal matrices are determined by the data it is desirable to have an algorithm which computes them to their inherent accuracy. In [DK88] such an algorithm was provided for computing the singular values to high relative accuracy. This new algorithm is a hybrid of the standardTshifted QR algorithm in [GK65ГBDMS79] Tand a new Tstable implementation of QR with a zero shift. It was also demonstrated empirically that this new algorithm was about as fast $\Gamma$ and often much faster $\Gamma$ than the standard algorithm [BDMS79] $\Gamma$ which can only provide absolute error bounds on the singular values.

In this paper we will prove that the algorithm in [DK88] also computes the singular vectors of bidiagonal $B$ with an error bound depending on the relative gap as in $\left(\mathbf{B}^{\prime}\right)$. More precisely $\Gamma$ it will compute singular vectors $u_{i}$ and $v_{i}$ with an error bound $q(n) \varepsilon /$ relgap $_{i} \Gamma$ where $q(n)$ is a modest function of the dimension $n$ of $B \Gamma$ and $\varepsilon$ is the machine precision as above. Thus This algorithm computes all features of the SVD of a bidiagonal matrix to their inherent uncertainties. (Actually we will need to change one line in the algorithm 
of [DK88] Tbut this change will effect none of the numerical or timing results reported in [DK88]. We will discuss this change further in sections 3 and 6 .)

If the bidiagonal matrix $B$ is the result of reducing a dense matrix $A$ to bidiagonal form $\Gamma$ then in general the entries of $B$ will have uncertainties of order $\varepsilon\|A\| \Gamma$ thus limiting the accuracy of the overall computation to bounds (A) and (B). A high accuracy SVD algorithm for dense matrices can be found in [DV90]. On situation where the reduction to bidiagonal form is accurate is the symmetric positive definite eigenvalue problem [BD88] $\Gamma$ where $B^{T} B=A$ is the Cholesky decomposition of $A$ Tso the eigenvalues of $A$ are the squares of the singular values of $B$.

The proof that the algorithm computes singular vectors as accurately as claimed has two new parts: bounding the error due to the stopping criterion $\Gamma$ and bounding the error due to roundoff in the zero-shift QR iteration. The stopping criterion bounds are similar to the bounds in $\left(\mathbf{A}^{\prime}\right)$ and $\left(\mathbf{B}^{\prime}\right)$ Tand are obtained via a specialized perturbation argument in which the SVD problem for a bidiagonal matrix is converted into an eigenvalue problem for a tridiagonal matrix with zero diagonalTfollowing [GVL83] (see section 4). On the other handI bounds for the roundoff errors due to repeated iterations of the algorithm are conveniently analyzed in terms of the long time behavior of a Hamiltonian differential equation on the space of matrices naturally associated with the algorithm ([DLT89ГSym82 ГChu86]).

To proceed $\Gamma$ we need to introduce some notation. QR iteration with a zero shift applied to a general invertible matrix $A_{0}$ produces a sequence of orthogonally similar matrices $A_{i}$ as follows. Given $A_{i} \Gamma$ compute its $Q R$ decomposition $A_{i}=Q R \Gamma$ where $Q$ is orthogonal and $R$ is upper triangular with positive diagonal. Then $A_{i+1}=R Q=Q^{T} A_{i} Q$. It is well known that if $A_{0}$ has eigenvalues with distinct moduliTthen $A_{i}$ converges to a triangular matrix with the eigenvalues on the diagonal as $i \rightarrow \infty$. This algorithm may be applied to the bidiagonal singular value problem as follows [GVL83]. Let $B_{0}$ be our initial bidiagonal matrix. Given $B_{i}$ T compute the $Q R$ decompositions $B_{i} B_{i}^{T}=Q_{1} R_{1}$ and $B_{i}^{T} B_{i}=Q_{2} R_{2}$. Then let $B_{i+1}=Q_{1}^{T} B_{i} Q_{2}$. Then $B_{i}$ is bidiagonal for all $i$ and converges as $i \rightarrow \infty$ to a diagonal matrix with the singular values on the diagonal. Observe that $B_{i+1} B_{i+1}^{T}=R_{1} Q_{1}$ and $B_{i+1}^{T} B_{i+1}=R_{2} Q_{2}$ Tso that the above zero-shift $S V D$ algorithm implicitly applies the usual QR iteration to $B_{i} B_{i}^{T}$ and $B_{i}^{T} B_{i}$ simultaneously.

We think of the SVD algorithm as a mapping from $\mathbf{R}^{2 n-1}$ (the entries of $B_{i}$ ) to $\mathbf{R}^{2 n-1}$ (the entries of $B_{i+1}$ ). To understand how errors propagate through iterations of the SVD algorithm Tit is natural to look at the Jacobian of this map Tsince the Jacobian describes how small perturbations in $B_{i}$ affect $B_{i+1}$. However $\Gamma$ since we are interested in the propagation of relative errors Twe will look instead at a Jacobian which maps small relative perturbations in $B_{i}$ to relative perturbations in $B_{i+1}$. To this end $\Gamma$ we will work with the logarithms of the entries $B_{i}$ and $B_{i+1}$ Tsince small perturbations in the logarithms of the matrix entries are equivalent to small relative perturbations in the matrix entries themselves. Thus $\Gamma$ we will think of a bidiagonal $B$ as a point in $\mathbf{R}^{2 n-1}$ through the identification (recall that the 
nontrivial entries of $B$ are positive)

$$
\left[\begin{array}{cccc}
a_{1} & b_{1} & & \\
& \ddots & \ddots & \\
& & \ddots & b_{n-1} \\
& & & a_{n}
\end{array}\right] \Longleftrightarrow\left[\begin{array}{c}
\log b_{1} \\
\vdots \\
\log b_{n-1} \\
\log a_{1} \\
\vdots \\
\log a_{n}
\end{array}\right]
$$

and think of one step of the SVD algorithm as a map $F$ which maps vectors of logarithms of entries of $B_{i}$ to vectors of logarithms of entries of $B_{i+1} \Gamma i=0,1,2, \ldots$. Thus for $j>i$

$$
F^{(j-i)}\left(B_{i}\right)=\underbrace{F \circ \cdots \circ F}_{j-i \text { times }}\left(B_{i}\right)=B_{j}
$$

We will call its Jacobian $M(j, i)$ Twhich by the chain rule is the product of the one step Jacobians $M(j, i)=M(j, j \Leftrightarrow 1) \cdots M(i+1, i)$. It is $M(j, i)$ which describes how initial relative errors in $B$ and roundoff errors committed during prior SVD iterations propagate during later SVD iterations.

The following four facts were observed during initial numerical experiments:

Fact 1: The eigenvalues of $M(j, i)$ appear in reciprocal pairs. In other words $\Gamma$ if $\lambda$ is an eigenvalue $\mathrm{s}$ so is $1 / \lambda$.

Fact 2: Near convergence (i.e. for $i$ large enough) $\Gamma$ the eigenvalues of $M(i+1, i)$ are simple $\Gamma$ approach 1 and all lie on the unit circle.

Fact 3: As $i \rightarrow \infty \Gamma M(i+1, i)$ converges to the constant matrix

$$
M_{\infty}=\left[\begin{array}{cc}
I_{n-1} & \Gamma_{n} \\
0 & I_{n}
\end{array}\right] \text { where } \Gamma_{n}=\left[\begin{array}{cccc}
\Leftrightarrow 2 & 2 & & \\
& \ddots & \ddots & \\
& & \Leftrightarrow 2 & 2
\end{array}\right]
$$

independent of initial data.

Fact 4: $\|M(j, i)\|$ grows linearly in the number of SVD steps $j \Leftrightarrow i$.

More precisely $\Gamma$ we observed numerically for a large class of problems that $\|M(j, i)\|_{\infty} \leq 5.06 \cdot n \cdot(j \Leftrightarrow i)$ ( $n$ is the matrix dimension). In section $9 \Gamma$ using O.D.E. methods $\Gamma$ we will prove $\|M(j, i)\|_{\infty} \leq(8 n \Leftrightarrow 4)(j \Leftrightarrow i)+O(1)$. This is the essential property of roundoff error propagation which lets us prove that the algorithm computes singular vectors as accurately as claimed. This linear growth is to be expected because near convergence we have

$$
M(j, i) \approx M_{\infty}^{j-i}=\left[\begin{array}{cc}
I_{n-1} & (j \Leftrightarrow i) \Gamma_{n} \\
0 & I_{n}
\end{array}\right]
$$

which grows linearly in norm. In section 10 we sketch an alternate proof of Fact 4 based on this intuition. 
In contrast $\Gamma$ a straightforward error analysis without the machinery developed in this paper would yield an error bound growing exponentially in $n$.

As we will see Facts 1 and $\mathbf{2}$ follow from the observation that linear combinations of the variables $\log b_{1}, \ldots, \log a_{n}$ satisfy a Hamiltonian differential equation with Hamiltonian $\Leftrightarrow \operatorname{tr}\left(\log \left(B B^{T}\right)^{2}\right) / 4$. The relationship between the flow and the algorithm $\cdots \rightarrow B_{i} \rightarrow$ $B_{i+1} \rightarrow \cdots$ is as follows: if $\log b_{1}(t), \ldots, \log a_{n}(t)$ solve the Hamiltonian flow with initial conditions $\log b_{1}^{(0)}, \ldots, \log a_{n}^{(0)}$ Tthen

$$
\left[\begin{array}{c}
\log b_{1}(i) \\
\vdots \\
\log a_{n}(i)
\end{array}\right]=\left[\begin{array}{c}
\log b_{1}^{(i)} \\
\vdots \\
\log a_{n}^{(i)}
\end{array}\right]
$$

where

$$
B_{i}=\left[\begin{array}{cccc}
a_{1}^{(i)} & b_{1}^{(i)} & & \\
& \ddots & \ddots & \\
& & \ddots & b_{n-1}^{(i)} \\
& & & a_{n}^{(i)}
\end{array}\right]
$$

gives the $i$-th step in the SVD algorithmTstarting from

$$
B_{0}=\left[\begin{array}{cccc}
a_{1}^{(0)} & b_{1}^{(0)} & & \\
& \ddots & \ddots & \\
& & \ddots & b_{n-1}^{(0)} \\
& & & a_{n}^{(0)}
\end{array}\right]
$$

(see sections 7 and 8 below). In contrast to many eigenvalue algorithms (see [Sym82T DLT 89]) Twhere the underlying symplectic structures are Lie-Poisson structuresThere the underlying structure is a so called Sklyanin structure [Sem84]. The variables $\log a_{1} \Gamma \log a_{1} a_{2}=$ $\log a_{1}+\log a_{2} \Gamma \ldots \Gamma \log a_{1} \cdots a_{n-1}=\log a_{1}+\cdots+\log a_{n-1}$ will turn out to be the momentum variables (note that $\log a_{n}$ does not appear because it is determined by the other variables through the relation $a_{1} \cdots a_{n}=\operatorname{det}(B)=$ constant)Tand the $\log b_{i}$ will be the position variables. In the limitr the momenta converge to constants (the sums of the logarithms of the singular values) and the positions move at constant speed toward $\Leftrightarrow \infty$ (i.e. the offdiagonals $b_{i}$ decay to zero geometrically).

Fact 1 will follow from the fact that the Jacobian with respect to the canonical Hamiltonian variables is symplectic; symplectic matrices have eigenvalues appearing in reciprocal pairs. Facts 2, 3 and 4 will follow from the asymptotics of the Hamiltonian system $F$ Fact 1Tand Krein's theory of strongly stable symplectic matrices [Kre50ГKre55].

The use of the differential equation as outlined above suggests a paradigm for seeking algorithms to solve other eigenvalue problems to high relative accuracy. The symplectic interpretation of the (fortuitously chosen) relative errors is that they correspond to perturbations in the canonical variables for the symplectic structure in which the SVD algorithm is Hamiltonian. The general paradigm we suggest is the following: given a Poisson structure in which a given eigenvalue algorithm is HamiltonianTone should try to construct natural 
global canonical variables (i.e. a global Darboux coordinate system). Such variables would indicate which functions of the eigenvalues (in our case their logarithms) are relatively insensitive to appropriate perturbations in the matrix (in our caseTrelative perturbations in the entries) and hence are computable to high accuracy.

The rest of the paper is organized as follows. Section 2 proves the perturbation results $\left(\mathbf{A}^{\prime}\right)$ and $\left(\mathbf{B}^{\prime}\right)$. Section 3 describes the algorithm $\mathrm{T}$ and section 4 bounds the error in the singular values and vectors introduced by its stopping criterion. Section 5 uses the bound on $\|M(j, i)\|$ (Fact 4$)$ to prove the error bounds for the zero-shift QR algorithm. Section 6 proves the main numerical result of the paper $\Gamma$ an error bound for the singular vectors computed by the overall algorithm. Section 7 describes flows and the SVD algorithm. It also provides an independent proof of the convergence of the zero-shift SVD algorithm with detailed (and we believe new) asymptotic expressions for the matrix entries. Section 8 discusses the Hamiltonian structure of the flow and proves Fact 1 above. Section 9 analyzes the asymptotics of $\|M(j, i)\|$ and proves Fact 4 . Section 10 discusses the spectrum of the one-step Jacobian of the SVDTand proves Facts 2 and 3. Section 11 presents numerical experiments $\Gamma$ and section 12 draws our conclusions.

We note that an alternative approach to computing singular values using gradient flows is presented in [Dri87].

Caveat: We will abuse the word "algorithm" in several differents ways. Sometimes it will refer to one step of the QR iteration (with or without shift) and sometimes it will refer to the full implementation with stopping criteria (the conventional [BDMS79] or the new [DK88] one). Which one is meant will be clear from context. 


\section{Perturbation Theory for Singular Vectors}

In this section we prove the perturbation bound (1.8) Twhich says that small relative perturbations in the entries of a bidiagonal matrix perturb the singular vectors by an amount proportional to the reciprocal of the relative gap (1.7). This result was conjectured in [DK88] Tand a weaker result proven in [BD88]. For the reader's convenience we also include a (new) proof of the eigenvalue bound (1.6).

The proofs depend on the following standard transformation [GK65]. Suppose the bidiagonal matrix $B$ has entries

$$
\left(\begin{array}{cccccc}
s_{1} & s_{2} & & & & \bigcirc \\
& s_{3} & s_{4} & & & \\
& & & \ddots & & \\
& & & & s_{2 n-3} & s_{2 n-2} \\
& & & & & s_{2 n-1}
\end{array}\right)
$$

and SVD $B=U \Sigma V^{T}$ Twith $\Sigma=\operatorname{diag}\left\{\sigma_{1}, \ldots, \sigma_{n}\right\} \Gamma V=\left[v_{1}, \ldots, v_{n}\right]$ and $U=\left[u_{1}, \ldots, u_{n}\right]$. Then the symmetric matrix

$$
S=\left(\begin{array}{lllll}
0 & s_{1} & & & \bigcirc \\
s_{1} & 0 & s_{2} & & \\
& s_{2} & 0 & & \ddots \\
& & \ddots & & s_{2 n-1} \\
& & & s_{2 n-1} & 0
\end{array}\right)
$$

has eigenvalues $\pm \sigma_{i}$ with normalized Tassociated eigenvectors

$$
h_{i}^{ \pm} \equiv 2^{-1 / 2}\left(v_{i 1}, \pm u_{i 1}, v_{i 2}, \pm u_{i 2}, \ldots, v_{i n}, \pm u_{i n}\right)^{T} .
$$

(Note for future reference that the components of $h_{i}^{ \pm}$are bounded by $1 / \sqrt{2}$.) Thus 5 the eigendecomposition for $S$ also yields the SVD for $B \Gamma$ and so perturbation theory for the eigenproblem for $S$ also computes perturbation theory for the SVD of $B$.

As described in [GvL]T the transformation $B \mapsto S$ should be viewed as the result of composing the SVD $\rightarrow$ eigenproblem map $\Gamma$

$$
B \mapsto\left(\begin{array}{ll}
0 & B^{T} \\
B & 0
\end{array}\right),
$$

with a perfect shuffle of the rows and columns $\Gamma\{1,2,3, \ldots, 2 n\} \rightarrow\{1, n+1,2, n+2, \ldots, n, 2 n\} \Gamma$ taking

$$
\left(\begin{array}{ll}
0 & B^{T} \\
B & 0
\end{array}\right) \mapsto S .
$$

Our first result bounds the effect of infinitesimal relative perturbations in the entries of $S$; the second result generalizes to finite perturbations.

Recall the standard fact that the eigenvalues of a tridiagonal matrix with nonzero off-diagonal entries are simple $\mathrm{C}$ and hence that the eigenvalues and eigenvectors depend smoothly on the entries of the matrix. 
Theorem 2.3 Let $S(t)$ be a matrix of the form (2.2), but with entries $s_{i}(t)$ which are smooth, positive functions of $t$. Let $\pm \sigma_{i}(t)$ and $h_{i}^{ \pm}(t)$ denote the eigenvalues and eigenvectors of $S(t)$ respectively. Then

$$
\left|\frac{\dot{\sigma}_{i}(0)}{\sigma_{i}(0)}\right| \leq(2 n \Leftrightarrow 1)\left(\max _{m}\left|\frac{\dot{s}_{m}(0)}{s_{m}(0)}\right|\right)
$$

and

$$
\left\|\dot{h}_{i}^{ \pm}(0)\right\| \leq(2 n \Leftrightarrow 1)\left(\max _{m}\left|\frac{\dot{s}_{m}(0)}{s_{m}(0)}\right|\right)\left(\frac{1}{\text { relgap }_{i}}\right) .
$$

Proof. Let

$$
\begin{aligned}
& \left\{\mu_{1}, \mu_{2}, \mu_{3}, \mu_{4}, \ldots\right\}=\left\{+\sigma_{1}, \Leftrightarrow \sigma_{1},+\sigma_{2}, \Leftrightarrow \sigma_{2}, \ldots\right\} \\
& \left\{\omega_{1}, \omega_{2}, \omega_{3}, \omega_{4}, \ldots\right\}=\left\{h_{1}^{+}, h_{1}^{-}, h_{2}^{+}, h_{2}^{-}, \ldots\right\}
\end{aligned}
$$

denote the eigenvalues and associated eigenvectors of $S$. Then $\Gamma$ by regular perturbation theory $\mathrm{Tand}$ repeated use of the eigenvalue equation $\Gamma$

$$
\begin{aligned}
\dot{\mu}_{i} & =\left(\omega_{i}, \dot{S} \omega_{i}\right)=\sum_{j=1}^{2 n-1}\left(\frac{2 \dot{s}_{j}}{s_{j}}\right)\left(\omega_{i}(j) s_{j} \omega_{i}(j+1)\right) \\
& =2 \sum_{j=1}^{2 n-1} \frac{\dot{s}_{j}}{s_{j}} \omega_{i}(j)\left(\mu_{i} \omega_{i}(j) \Leftrightarrow s_{j-1} \omega_{i}(j \Leftrightarrow 1)\right) \\
& =\cdots=2 \mu_{i} \sum_{j=1}^{2 n-1} \frac{\dot{s}_{j}}{s_{j}}\left(\sum_{m=1}^{j} \omega_{i}^{2}(m)(\Leftrightarrow 1)^{j-m}\right)
\end{aligned}
$$

Thus

$$
\left|\frac{\dot{\mu}_{i}}{\mu_{i}}\right| \leq 2\left(\max _{k}\left|\frac{\dot{s}_{k}}{s_{k}}\right|\right) \sum_{j=1}^{2 n-1}\left|\sum_{m=1}^{j} \omega_{i}^{2}(m)(\Leftrightarrow 1)^{j-m}\right| .
$$

On the other handlit follows from (2.6) and the perpendicularity of $h_{q}^{+}$and $h_{q}^{-}$Tthat

$$
\sum_{m=1}^{n} \omega_{i}^{2}(2 m)=\sum_{m=1}^{n} \omega_{i}^{2}(2 m \Leftrightarrow 1)=\frac{1}{2}\left(\sum_{m=1}^{2 n} \omega_{i}^{2}(m)\right)=\frac{1}{2} .
$$

But then

$$
\left|\sum_{m=1}^{j} \omega_{i}^{2}(m)(\Leftrightarrow 1)^{j-m}\right| \leq \frac{1}{2}
$$

which proves (2.4).

Again by regular perturbation theory and repeated use of the eigenvalue equation $\Gamma$

$$
\dot{\omega}_{i}=\sum_{k \neq i}\left(\omega_{k}, \dot{S} \omega_{i}\right) \frac{\omega_{k}}{\mu_{i} \Leftrightarrow \mu_{k}}
$$




$$
\begin{gathered}
=\sum_{m=1}^{2 n-1}\left(\frac{\dot{s}_{m}}{s_{m}}\right) \sum_{k \neq i}\left(\omega_{k}(m) s_{m} \omega_{i}(m+1)+\omega_{i}(m) s_{m} \omega_{k}(m+1)\right) \frac{\omega_{k}}{\mu_{i} \Leftrightarrow \mu_{k}} \\
=\sum_{m=1}^{2 n-1}\left(\frac{\dot{s}_{m}}{s_{m}}\right) \sum_{k \neq i}\left(\omega_{k}(m) \omega_{i}(m)\left(\mu_{i}+\mu_{k}\right) \Leftrightarrow \omega_{i}(m \Leftrightarrow 1) s_{m-1} \omega_{k}(m)\right. \\
\left.\Leftrightarrow \omega_{k}(m \Leftrightarrow 1) s_{m-1} \omega_{i}(m)\right) \frac{\omega_{k}}{\mu_{i} \Leftrightarrow \mu_{k}} \\
=\cdots=\sum_{m=1}^{2 n-1}\left(\frac{\dot{s}_{m}}{s_{m}}\right) \sum_{k \neq i}\left(\frac{\mu_{i}+\mu_{k}}{\mu_{i} \Leftrightarrow \mu_{k}}\right)\left(\sum_{\ell=1}^{m} \omega_{k}(\ell) \omega_{i}(\ell)(\Leftrightarrow 1)^{m-\ell}\right) \omega_{k} .
\end{gathered}
$$

Using the orthonormality of the eigenvectors $\omega_{k}$ Twe obtain

$$
\begin{aligned}
\left\|\dot{\omega}_{i}\right\| & \leq\left(\max _{m}\left|\frac{\dot{s}_{m}}{s_{m}}\right|\right) \sum_{m=1}^{2 n-1}\left\|\sum_{k \neq i}\left(\frac{\mu_{i}+\mu_{k}}{\mu_{i} \Leftrightarrow \mu_{k}}\right)\left(\sum_{\ell=1}^{m} \omega_{k}(\ell) \omega_{i}(\ell)(\Leftrightarrow 1)^{m-\ell}\right) \omega_{k}\right\| \\
& =\left(\max _{m}\left|\frac{\dot{s}_{m}}{s_{m}}\right|\right) \sum_{m=1}^{2 n-1}\left(\sum_{k \neq i}\left(\frac{\mu_{i}+\mu_{k}}{\mu_{i} \Leftrightarrow \mu_{k}}\right)^{2}\left(\sum_{\ell=1}^{m} \omega_{k}(k) \omega_{i}(\ell)(\Leftrightarrow 1)^{m-\ell}\right)^{2}\right)^{1 / 2} \\
& \leq\left(\max _{m}\left|\frac{\dot{s}_{m}}{s_{m}}\right|\right)\left(\max _{k \neq i}\left|\frac{\mu_{i}+\mu_{k}}{\mu_{i} \Leftrightarrow \mu_{k}}\right|\right) \sum_{m=1}^{2 n-1}\left(\sum_{k=1}^{2 n}\left(\sum_{\ell=1}^{m} \omega_{k}(\ell) \omega_{i}(\ell)(\Leftrightarrow 1)^{m-\ell}\right)^{2}\right)^{1 / 2}
\end{aligned}
$$

But again $\Gamma$ by orthonormality $\Gamma$

$$
\begin{aligned}
\sum_{k=1}^{2 n}\left(\sum_{\ell=1}^{m} \omega_{k}(\ell) \omega_{i}(\ell)(\Leftrightarrow 1)^{m-\ell}\right)^{2} & =\sum_{1 \leq \ell, q \leq m}\left(\sum_{k=1}^{2 n} \omega_{k}(\ell) \omega_{k}(q) \omega_{i}(\ell) \omega_{i}(q)(\Leftrightarrow 1)^{m-\ell}(\Leftrightarrow 1)^{m-q}\right) \\
& =\sum_{\ell=1}^{m} \omega_{i}^{2}(\ell) \leq 1 .
\end{aligned}
$$

A simple computation shows that if $\mu_{i}= \pm \sigma_{i^{\prime}}$ Tthen $\max _{k \neq i}\left|\frac{\mu_{i}+\mu_{k}}{\mu_{i}-\mu_{k}}\right|=\left(\text { rel } \text { gap }_{i^{\prime}}\right)^{-1}$. This proves (2.5) Tand the Theorem.

Remark. From $(2.3) \Gamma|| \dot{h}_{i}^{ \pm} \|^{2}=\frac{1}{2}\left(\left\|\dot{u}_{i}\right\|^{2}+\left\|\dot{v}_{i}\right\|^{2}\right)$. Hence (2.5) yields

$$
\max \left(\left\|\dot{u}_{i}\right\|,\left\|\dot{v}_{i}\right\|\right) \leq \sqrt{2}(2 n \Leftrightarrow 1)\left(\max _{m}\left|\frac{\dot{s}_{m}(0)}{s_{m}(0)}\right|\right)\left(\frac{1}{\text { relgap }_{i}}\right) .
$$

We also have the following global error bound.

Theorem 2.12 Let $B$ and $B^{\prime}$ be bidiagonal matrices with positive entries $B_{i k}$ and $B_{i k}^{\prime}$. Let

$$
\eta_{r} \equiv(2 n \Leftrightarrow 1) \max _{j, k}\left|\log \frac{B_{j k}^{\prime}}{B_{j k}}\right|
$$

be the relative error in $B$ as defined in (1.5), and $\eta_{r}^{\prime} \equiv e^{\eta_{r}} \Leftrightarrow 1>\eta_{r}>0$. Then

$$
1 \Leftrightarrow \eta_{r}^{\prime}<e^{-\eta_{r}}<\frac{\sigma_{i}^{\prime}}{\sigma_{i}}<e^{\eta_{r}}=1+\eta_{r}^{\prime}
$$


which implies

$$
\Leftrightarrow \eta_{r}^{\prime}<\frac{\sigma_{i}^{\prime} \Leftrightarrow \sigma_{i}}{\sigma_{i}}<\eta_{r}^{\prime}
$$

Furthermore, if $\eta_{r}^{\prime}<$ relgap $_{i}$, the sines of the angles $\theta\left(u_{i}, u_{i}^{\prime}\right), \theta\left(v_{i}, v_{i}^{\prime}\right)$ between the unperturbed singular vectors $u_{i}, v_{i}$ and the perturbed singular vectors $u_{i}^{\prime}, v_{i}^{\prime}$, are bounded by

$$
\max \left(\sin \theta\left(u_{i}, u_{i}^{\prime}\right), \sin \theta\left(v_{i}, v_{i}^{\prime}\right)\right) \leq \frac{\sqrt{2} \eta_{r}^{\prime}\left(1+\eta_{r}^{\prime}\right)}{\operatorname{relgap}_{i} \Leftrightarrow \eta_{r}^{\prime}}
$$

Proof. Let $S$ and $S^{\prime}$ be matrices of the form (2.2) derived from $B$ and $B^{\prime}$ as before. Set $S(t)=S+t\left(S^{\prime} \Leftrightarrow S\right) \Gamma 0 \leq t \leq 1$. With the notation of Theorem 2.3 Wwe have from $(2.7)$ and $(2.9) \Gamma$

$$
\begin{aligned}
\left|\log \frac{\mu_{i}^{\prime}}{\mu_{i}}\right| & \leq \int_{0}^{t}\left|\frac{d}{d t} \log \mu_{i}(t)\right| d t \\
& \leq \sum_{j=1}^{2 n-1}\left(\int_{0}^{1}\left|\frac{\dot{s}_{j}}{s_{j}}\right| d t\right) \\
& =\sum_{j=1}^{2 n-1}\left|\log \frac{s_{j}^{\prime}}{s_{j}}\right| \\
& \leq \eta_{r}
\end{aligned}
$$

which proves (2.14). Note that the eigenvalues of $s(t)$ cannot pass through zero as $t$ varies from zero to one.

In a similar waylfrom (2.10) and the calculation that follows in Theorem $2.3 \Gamma$

$$
|| \dot{\omega}_{i} \| \leq\left(\max _{0 \leq t \leq 1} \max _{k \neq i}\left|\frac{\mu_{i}(t)+\mu_{k}(t)}{\mu_{i}(t) \Leftrightarrow \mu_{k}(t)}\right|\right) \sum_{m=1}^{2 n-1}\left|\frac{\dot{s}_{m}(t)}{s_{m}(t)}\right|,
$$

which yields

$$
\left\|w_{i}^{\prime} \Leftrightarrow w_{i}\right\| \leq\left(\max _{0 \leq t \leq 1} \max _{k \neq i}\left|\frac{\mu_{i}(t)+\mu_{k}(t)}{\mu_{i}(t) \Leftrightarrow \mu_{k}(t)}\right|\right) \eta_{r}
$$

and hence

$$
\left\|\left(h_{i}^{ \pm}\right)^{\prime} \Leftrightarrow h_{i}^{ \pm}\right\| \leq\left(\max _{0 \leq t \leq 1} \max _{k \neq i}\left|\frac{\sigma_{i}(t)+\sigma_{k}(t)}{\sigma_{i}(t) \Leftrightarrow \sigma_{k}(t)}\right|\right) \eta_{r},
$$

as noted at the end of the proof of Theorem 2.3.

Now observe that

$$
\eta_{r}(t)=(2 n \Leftrightarrow 1) \max _{j}\left|\log \frac{s_{j}(t)}{s_{j}(0)}\right| \leq \eta_{r}
$$

for all $0 \leq t \leq 1$. Thus from $(2.14)$

$$
1 \Leftrightarrow \eta_{r}^{\prime}<e^{-\eta_{r}} \leq \frac{\sigma_{i}(t)}{\sigma_{i}(0)} \leq e^{\eta_{r}}=1+\eta_{r}^{\prime} .
$$

Suppose $\sigma_{i}(t)>\sigma_{k}(t)>0$. Then

$$
\sigma_{i}(t)+\sigma_{k}(t) \leq e^{\eta_{r}}\left(\sigma_{i}(0)+\sigma_{k}(0)\right),
$$


and

$$
\begin{aligned}
\sigma_{i}(t) \Leftrightarrow \sigma_{k}(t) & \geq \sigma_{i}(0)\left(1 \Leftrightarrow \eta_{r}^{\prime}\right) \Leftrightarrow \sigma_{k}(0)\left(1+\eta_{r}^{\prime}\right) \\
& =\left[\left(\frac{\sigma_{i}(0) \Leftrightarrow \sigma_{k}(0)}{\sigma_{i}(0)+\sigma_{k}(0)}\right) \Leftrightarrow \eta_{r}^{\prime}\right]\left(\sigma_{i}(0)+\sigma_{k}(0)\right) \\
& \geq\left(\text { relgap }_{i} \Leftrightarrow \eta_{r}^{\prime}\right)\left(\sigma_{i}(0)+\sigma_{k}(0)\right),
\end{aligned}
$$

so that for $\eta_{r}^{\prime}<\operatorname{relgap}_{i} \Gamma$

$$
\left|\frac{\sigma_{i}(t)+\sigma_{k}(t)}{\sigma_{i}(t) \Leftrightarrow \sigma_{k}(t)}\right| \leq \frac{e^{\eta_{r}}}{r e \operatorname{lgap} p_{i} \Leftrightarrow \eta_{r}^{\prime}}=\frac{1+\eta_{r}^{\prime}}{\operatorname{relgap}_{i} \Leftrightarrow \eta_{r}^{\prime}} .
$$

The same inequality holds if $\sigma_{k}(t)>\sigma_{i}(t)$ Tand we obtain

$$
\left\|\left(h_{i}^{ \pm}\right)^{\prime} \Leftrightarrow\left(h_{i}^{ \pm}\right)\right\| \leq \frac{\eta_{r}\left(1+\eta_{r}^{\prime}\right)}{\operatorname{relgap}_{i} \Leftrightarrow \eta_{r}^{\prime}} .
$$

As

$$
\begin{aligned}
& \sin \theta\left(u_{i}, u_{i}^{\prime}\right) \leq\left\|u_{i}^{\prime} \Leftrightarrow u_{i}\right\| \leq \sqrt{2}\left\|\left(h_{i}^{+}\right)^{\prime} \Leftrightarrow h_{i}^{+}\right\| \\
& \sin \theta\left(v_{i}, v_{i}^{\prime}\right) \leq\left\|v_{i}^{\prime} \Leftrightarrow v_{i}\right\| \leq \sqrt{2}\left\|\left(h_{i}^{+}\right)^{\prime} \Leftrightarrow h_{i}^{+}\right\|,
\end{aligned}
$$

and as $\eta_{r}<\eta_{r}^{\prime}$ Tthe result follows. 


\section{The SVD Algorithm}

In this section we describe the algorithm for the bidiagonal SVD. This algorithm was introduced in [DK88] Tand discussed in detail there. HereTwe present a simplified version of the algorithm which is adequate to prove our error bounds. The practical enhancements we omit here can greatly improve performance in some cases $\Gamma$ but will not invalidate our error analysis. It turns out our eventual error bounds will depend on the number of QR steps necessary for convergence; the practical enhancements often reduce this number dramatically and we summarize our numerical experience with the number of QR steps required in section 11.

Briefly Tthe algorithm is a hybrid of the standard shifted $\mathrm{QR}$ algorithm and the implicit zero-shift $Q R$ algorithm. The standard shifted QR is used on matrices which are wellconditioned $\left(\sigma_{n}\right.$ is not much smaller than $\left.\sigma_{1}\right) \Gamma$ and implicit zero-shift QR is used on illconditioned submatrices $\left(\sigma_{n} \ll \sigma_{1}\right)$. Implicit zero-shift $\mathrm{QR}$ is much more accurate than shifted QRT but much slower if the matrix is well-conditioned. Fortunately shifted QR is adequately accurate on well-conditioned matrices $\Gamma$ so we only need to exploit the more accurate implicit zero-shift QR when it is also fast. Thus $\mathrm{Q}$ as the algorithm runs the offdiagonal entries decrease and are eventually set to zero $\Gamma$ deflating the matrix. On each newly deflated submatrix $\Gamma \sigma_{n}$ and $\sigma_{1}$ are cheaply but reliably estimated and either shifted QR or implicit zero-shift QR used depending on the ratio $\sigma_{n} / \sigma_{1}$.

We will need to change one line in the algorithm presented in [DK88] in order to prove our error bounds in section 6 . This change will not alter any of the numerical or timing results reported in [DK88].

We will present the implicit zero-shift $\mathrm{QR}$ algorithm $\Gamma$ the stopping criterion for setting tiny offdiagonal entries to zeroT and finally the overall algorithm. Implicit zero-shift $Q R$ calls the subroutine $\operatorname{ROT}(f, g, c s, s n, r)$ [GVL83] Twhich takes $f$ and $g$ as inputs and returns $r \Gamma c s=\cos \theta$ and $s n=\sin \theta$ such that

$$
\left[\begin{array}{cc}
c s & s n \\
\Leftrightarrow s n & c s
\end{array}\right] \cdot\left[\begin{array}{l}
f \\
g
\end{array}\right]=\left[\begin{array}{l}
r \\
0
\end{array}\right]
$$

$\mathbf{R O T}(\mathbf{f}, \mathbf{g}, \mathbf{c s}, \mathbf{s n}, \mathbf{r})$ : take $f$ and $g$ as input and returns $c s \Gamma s n$ and $r$ satisfying (3.1).

$$
\begin{aligned}
& \text { if } \quad \begin{array}{l}
(f=0) \text { then } \\
c s=0 ; s n=1 ; r=g ;
\end{array} \\
& \text { elseif }(|f|>|g|) \text { then } \\
& \quad t=g / f ; t t=\sqrt{1+t^{2}} \\
& \quad c s=1 / t t ; s n=t * c s ; r=f * t t \\
& \text { else } \quad \\
& \quad t=f / g ; t t=\sqrt{1+t^{2}} \\
& \quad s n=1 / t t ; c s=t * s n ; r=g * t t \\
& \text { endif }
\end{aligned}
$$


The algorithm also calls subroutine UPDATE $\left(c s, s n, v_{1}, v_{2}\right)$ :

UPDATE $\left(\mathbf{c s}, \mathbf{s n}, \mathbf{v}_{1}, \mathbf{v}_{2}\right)$ : replace $n$-vectors $v_{1}$ and $v_{2}$ by $c s \cdot v_{1}+s n \cdot v_{2}$ and $\Leftrightarrow s n \cdot v_{1}+c s \cdot v_{2}$.

$$
\text { for } \begin{array}{ll}
i=1 \text { to } n \\
& t=v_{1}(i) \\
& v_{1}(i)=c s * t+s n * v_{2}(i) \\
& v_{2}(i)=\Leftrightarrow s n * t+c s * v_{2}(i)
\end{array}
$$

endfor

Implicit Zero-Shift QR Algorithm: Let $B$ be an $n$ by $n$ bidiagonal matrix with diagonal entries $a_{1}, \ldots, a_{n}$ and superdiagonal entries $b_{1}, \ldots, b_{n-1}$. The following algorithm replaces $a_{i}$ and $b_{i}$ by new values corresponding to one step of the QR iteration with zero shift. It also updates the right unit singular vectors $v_{i}$ and left unit singular vectors $u_{i}$ (at the start of the algorithm $\Gamma$ these should be initialized to the columns of the identity matrix: $v_{i}(j)=u_{i}(j)=\delta_{i j}$.

$$
\begin{aligned}
& \text { oldcs }=1 \\
& f=a_{1} \\
& g=b_{1} \\
& \text { for } i=1 \Gamma n \Leftrightarrow 1 \\
& \text { call } \operatorname{ROT}(f, g, c s, s n, r) \\
& \text { call UDPATE }\left(c s, s n, v_{i}, v_{i+1}\right) \\
& \text { if }(i \neq 1) \Gamma b_{i-1}=\text { olds } n * r \\
& f=\text { oldcs } * r \\
& g=a_{i+1} * s n \\
& h=a_{i+1} * c s \\
& \text { call } R O T(f, g, c s, s n, r) \\
& \text { call UPDATE }\left(c s, s n, u_{i}, u_{i+1}\right) \\
& a_{i}=r \\
& f=h \\
& g=b_{i+1} \\
& \text { oldcs }=c s \\
& \text { oldsn }=s n
\end{aligned}
$$


This algorithm may also be expressed in the following terser but equivalent form (we will need the expanded form above for the analysis):

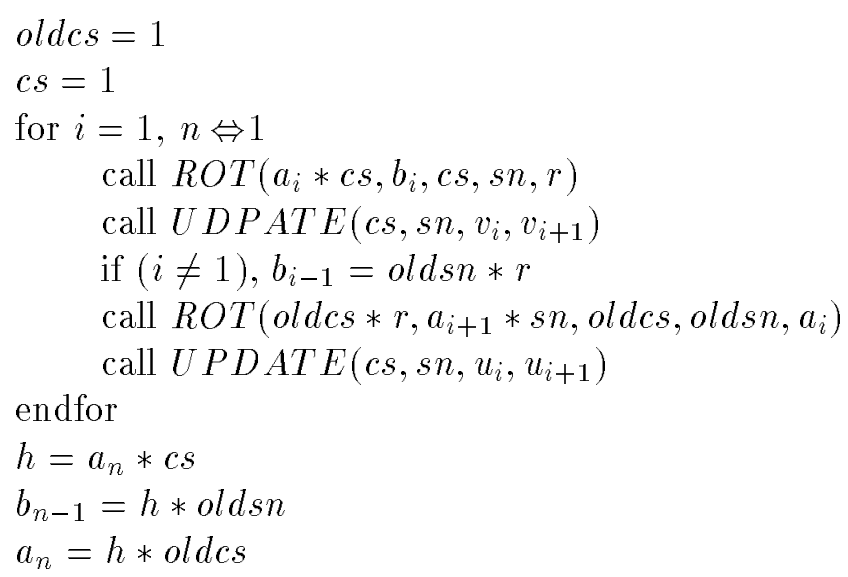

Next we discuss the stopping criterion $\Gamma i . e$. how to decide when to set an offdiagonal $b_{i}$ to zero and so converge. It is important that the stopping criterion introduce error in the singular vectors not much worse than the bound of Theorem 2.2 Ssince otherwise we will not compute the singular vectors to their inherent accuracy. The criterion described in [DK88] is the following.

Stopping Criterion: This algorithm decides when an offdiagonal entry $b_{i}$ can be set to zero. $0<$ tol $<1$ is a relative error tolerance.

$$
\begin{aligned}
& \mu_{1}=a_{1} \\
& \text { for } j=1 \Gamma n \Leftrightarrow 1 \\
& \quad \text { if }\left|b_{j}\right|<t o l * \mu_{j} \Gamma \text { set } b_{j}=0 \\
& \quad \mu_{j+1}=\left|a_{j+1}\right| *\left(\mu_{j} /\left(\mu_{j}+\left|b_{j}\right|\right)\right) \\
& \text { endfor }
\end{aligned}
$$

It was shown in [DK88] that this criterion perturbs the singular values of $B$ by no more than about $n \cdot$ tol $/ 2^{1 / 2}$ when $t o l \ll 1$. In sections $4 \Gamma 5$ and 6 we will show that it also has a small effect on the singular vectors Tin particular that it does not change them in direction

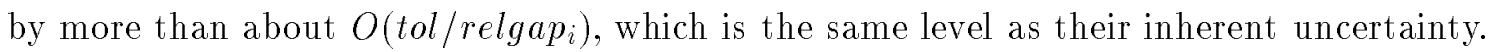


ThusTthe overall algorithm can be summarized as follows.

\section{Bidiagonal SVD Algorithm (simplified)}

Loop:

Find the bottommost unreduced submatrix of $B$; call it $\hat{B}$.

(Let $s$ and $e$ be the starting and ending indices of $\hat{B}$ within $B$.

Then $b_{e}=0$ if $e<n \Gamma b_{s-1}=0$ if $s>1$ and $b_{i} \neq 0$ for $s \leq i \leq e \Leftrightarrow 1$.)

If $\hat{B}$ is 1 by $1(s=e)$ Twe are done.

Apply the stopping criterion to $\hat{B}$; if any $b_{i}$ are set to 0Treturn to Loop

Estimate the smallest singular value $\underline{\sigma}$ and the largest singular value $\bar{\sigma}$ of $\hat{B}$.

if $n * \underline{\sigma} / \bar{\sigma}<\max (\varepsilon /$ tol, .01) then

Use implicit zero-shift $\mathrm{QR}$ on $\hat{B}$

else

Use standard shifted QR on $\hat{B}$

endif

\section{Goto Loop}

The difference between this algorithm and the one in [DK88] (besides some insignificant simplifications $)$ is the use of the test $(n * \underline{\sigma} / \bar{\sigma}<\max (\varepsilon /$ tol, .01$))$ in place of $(n * \underline{\sigma} / \bar{\sigma}<\varepsilon /$ tol $)$ to determine whether or to use zero-shift $\mathrm{QR}$ or shifted $\mathrm{QR}$. The reason for this change will become apparent in section 6 . In [DK88] the value of tol used in the numerical tests was tol $=100 * \varepsilon \Gamma$ so this change has no effect on the results reported there. The value 100 was chosen empirically to make the algorithm fast Tbut could easily be made as large as 1000 or as small as 10 without greatly impacting performance.

We summarize here the ways in which the above description simplifies the actual algorithm of [DK88] Targue that we have not omitted any features which could greatly increase the errorTand summarize the properties of the practical algorithm we do need for the error analysis.

Bidirectional $Q R$ : If $B$ is as given in (1.1) Twe define $\operatorname{rev}(B)$ as the matrix

$$
\operatorname{rev}(B)=\left[\begin{array}{cccc}
a_{n} & b_{n-1} & & \\
& \ddots & \ddots & \\
& & \ddots & b_{1} \\
& & & a_{1}
\end{array}\right],
$$

i.e. $B$ with the diagonals reversed. The SVDs of $B$ and $\operatorname{rev}(B)$ are simply related since $B=\operatorname{Prev}(B)^{T} P^{T}$ Twhere $P$ is the permutation matrix with ones going from the bottom left to the upper right: $B=U \Sigma V^{T}$ implies $\operatorname{rev}(B)=(P V) \Sigma(P U)^{T}$. It turns out that 
QR iteration may converge much faster applied to $\operatorname{rev}(B)$ than $B \Gamma$ and so the practical algorithm tries to exploit this and perform whichever one is faster. The reason for the speed difference is as follows. As zero-shift QR converges $T$ the singular values appear on the diagonal in decreasing order from upper left to lower right. If the entries of $B$ are already graded in this way the algorithm will converge more quickly than if they are not. The algorithm tests for this grading in a very simple way: if $\left|a_{1}\right|>\left|a_{n}\right| \Gamma \mathrm{QR}$ is applied to $B \Gamma$ and otherwise to $\operatorname{rev}(B)$. This is not a foolproof schemeTbut can quadruple the speed for strongly graded matrices.

Since the SVDs of $B$ and $\operatorname{rev}(B)$ are essentially permutations of one anotherTit suffices to perform an error analysis either of QR applied to $B$ or QR applied to $\operatorname{rev}(B)$. Our error bound will however depend on the total number of QR steps takenTand so benefit from the practical enhancements; we summarize our numerical experience with the number of $Q R$ steps required for convergence in the section 11. Of course in practice the number of QR steps is known after the algorithm terminates $\Gamma$ and this value could be used in our later error bounds.

Bidirectional stopping criterion: Just as QR can be applied either to $B$ or $\operatorname{rev}(B) \Gamma$ so can the stopping criterion. In fact we apply it to both $B$ and $\operatorname{rev}(B)$ Tno matter to which of the two we apply QR.

2 by 2 submatrices: When the bottommost unreduced submatrix $\hat{B}$ is 2 by $2 \Gamma$ we can apply the quadratic formula to directly compute its SVD. In practice $\mathrm{C}$ we implement it quite carefully so that when addition and subtraction are implemented with a guard digit $\left(f l(a \pm b)=(a \pm b)\left(1+\varepsilon_{1}\right) \Gamma\left|\varepsilon_{1}\right| \leq \varepsilon\right)$ we compute both the singular values and singular vectors to nearly full machine precision Teven if the relative gap is small so that the singular vectors are ill-conditioned. In the absence of a guard digit (the model of arithmetic in (5.1)) we only compute nearly the exact singular values and vectors of a matrix which differs from the input in a few bits in each entry. Thus Tthe global perturbation bounds of section 2 are respected.

Deflation when $a_{i}=0$ : When some $a_{i}=0 \Gamma$ then the code will automatically set $b_{i-1} \Gamma b_{n-1}$ and $a_{n}$ to zero; this is because $f=h=0$ at the end of each loop iteration. Thus This case needs no special consideration (in contrast to the standard SVD [BDMS79] which treats this case specially).

Choosing implicit zero-shift $Q R$ versus standard shifted $Q R$ : We need to compute cheap and reliable estimates of the smallest singular value $\underline{\sigma}$ and largest singular value $\bar{\sigma}$ in order to decide whether to use standard or implicit zero-shift QR. The largest singular value $\bar{\sigma}$ can clearly be estimated to within a factor of 2 by the largest absolute entry of $B$. The smallest singular value $\underline{\sigma}$ turns out to be estimated to within a factor of $n^{ \pm 1 / 2}$ by $\min _{k} \mu_{k} \Gamma$ where the $\mu_{k}$ are computed by the recurrence in the Stopping Criterion above [DK88]. This uncertainty in $\underline{\sigma}$ and $\bar{\sigma}$ will contribute a factor of $n^{1 / 2}$ to our final error bound. 


\section{Error Bounds for the Stopping Criterion}

Let $B$ be a bidiagonal matrix with positive entries $a_{p}, b_{q}$ Tand let $B^{\prime}$ be the bidiagonal matrix obtained from $B$ by setting $b_{j}=0$ for some $j \Gamma 1 \leq j \leq n \Leftrightarrow 1$. Let $S$ and $S^{\prime}$ be the associated tridiagonal matrices of form (2.2).

Define vectors $z_{2 j}, z_{2 j+1}$ through

$$
\begin{gathered}
S z_{2 j}=e_{2 j} \\
S z_{2 j+1}=e_{2 j+1},
\end{gathered}
$$

where $\left\{\boldsymbol{e}_{i}\right\}$ give the standard basis in $\boldsymbol{R}^{2 n}$. A simple calculation $\Gamma$ using the explicit form of $S$ Tshows that

$$
\begin{gathered}
z_{2 j}(\ell)=0 \quad \text { if } \quad \ell \text { is even or } \ell>2 j+1 \\
z_{2 j+1}(\ell)=0 \text { if } \ell \text { is odd } \quad \text { or } \ell<2 j
\end{gathered}
$$

Set

$$
\begin{aligned}
& m_{1} \equiv \min \left(s_{2 j}\left\|z_{2 j}\right\|_{1}, s_{2 j}\left\|z_{2 j+1}\right\|_{1}\right) \\
& m_{1}^{\prime} \equiv e^{m_{1}} \Leftrightarrow 1>m_{1} \\
& m_{2} \equiv \min \left(s_{2 j}\left\|z_{2 j}\right\|_{2}, s_{2 j}\left\|z_{2 j+1}\right\|_{2}\right) \\
& m_{2}^{\prime}=e^{m_{2}} \Leftrightarrow 1>m_{2},
\end{aligned}
$$

where $\|\cdot\|_{1},\|\cdot\|_{2}$ denote the $L^{1}$ and $L^{2}$ norms respectively. It is easy to see that for a given $j \Gamma$

$$
m_{1}=b_{j} \mu_{j}
$$

where $\mu_{j}$ appears in the Stopping Criterion of $\S 3$. Similarly

$$
m_{2}=b_{j} \eta_{j}
$$

corresponds to $\mu_{j} \Gamma$ but for rev $(B)$ as in (3.2). (See the discussion of the bidirectional stopping criterion in $\S 3$.)

Theorem 4.1 Let $B, B^{\prime}$ etc. be as above. Let $\sigma_{i}, \sigma_{i}^{\prime}$ be the singular values of $B, B^{\prime}$ respectively, and let $u_{i}, v_{i}$ and $u_{i}^{\prime}, v_{i}^{\prime}$ be the associated singular vectors, making angles $\theta\left(u_{i}, u_{i}^{\prime}\right), \theta\left(v_{i}, v_{i}^{\prime}\right)$ respectively. Then for $1 \leq i \leq n$,

$$
1 \Leftrightarrow m_{\ell}^{\prime}<e^{-m_{\ell}} \leq \frac{\sigma_{i}^{\prime}}{\sigma_{i}} \leq e^{m_{\ell}}=1+m_{\ell}^{\prime},
$$

where $\ell=1,2$.

Furthermore, if $m_{1}^{\prime}<$ relgap $_{i}$,

$$
\max \left(\sin \theta\left(u_{i}, u_{i}^{\prime}\right), \sin \theta\left(v_{i}, v_{i}^{\prime}\right)\right) \leq m_{1}^{\prime}\left(\frac{1}{\sqrt{2}}\left(1+\sqrt{n \Leftrightarrow \frac{1}{2}}\right)+\left(\frac{1+m_{1}^{\prime}}{\text { relgap }_{i} \Leftrightarrow m_{1}^{\prime}}\right) \sqrt{\frac{2 n+5}{4}}\right),
$$

and if $m_{2}^{\prime}<$ relgap,

$$
\max \left(\sin \theta\left(u_{i}, u_{i}^{\prime}\right), \sin \theta\left(v_{i}, v_{i}^{\prime}\right)\right) \leq m_{2}^{\prime}\left(1+\frac{\left(1+m_{2}^{\prime}\right)}{r e l g a p_{i} \Leftrightarrow m_{2}^{\prime}}\right) .
$$


Proof. Let $S(t)$ be the matrix obtained from $S$ by replacing $s_{2 j}$ by $t s_{2 j} \Gamma 0 \leq t \leq 1$. Note that $S(1)=S \Gamma S(0)=S^{\prime}$. By (4.1) and (4.2) Гnote that

$$
\begin{aligned}
& S(t) z_{2 j}=S z_{2 j}=e_{2 j} \\
& S(t) z_{2 j+1}=S z_{2 j+1}=e_{2 j+1}
\end{aligned}
$$

for all $t$ in the interval.

With the notation of Theorem 2.3Г

$$
\begin{aligned}
\dot{\mu}_{i}(t) & =2 s_{i j}\left(e_{2 j}, w_{i}(t)\right)\left(e_{2 j+1}, w_{i}(t)\right) \\
& =2 s_{i j}\left(S(t) z_{2 j}, w_{i}(t)\right)\left(e_{2 j+1}, w_{i}(t)\right) \\
& =2 s_{i j} \mu_{i}\left(z_{2 j}, w_{i}(t)\right)\left(e_{2 j+1}, w_{i}(t)\right) .
\end{aligned}
$$

But $\left|\left(e_{2 j+1}, w_{i}(t)\right)\right|=2^{-1 / 2}$ and $\left|\left(z_{2 j}, w_{i}(t)\right)\right| \leq\left\|z_{2 j}\right\|_{1}\left\|w_{i}(t)\right\|_{\infty} \leq\left\|z_{2 j}\right\|_{1} 2^{-1 / 2}$. On the other hand from $(4.3) \Gamma$

$$
\begin{aligned}
\left|\left(z_{2 j}, w_{i}(t)\right)\right| & \leq 2^{-1 / 2}\left\|z_{2 j}\right\|_{2}\left\|v_{i^{\prime}}(t)\right\|_{2}, \text { for suitable } i^{\prime}, \\
& =2^{-1 / 2}\left\|z_{2 j}\right\|_{2} .
\end{aligned}
$$

Thus

$$
\left|\frac{d \log \mu_{i}(t)}{d t}\right| \leq s_{2 j}\left\|z_{2 j}\right\|_{\ell}=m_{\ell}, \quad \ell=1,2
$$

and integration gives the desired eigenvalue bound $(4.7)_{\ell} \Gamma \ell=1,2$.

We now prove $(4.8)_{2}$. Perturbation theory gives

$$
\begin{aligned}
\frac{d w_{i}}{d t}= & \sum_{k \neq i}\left(w_{i}, \dot{S} w_{k}\right) \frac{w_{k}}{\mu_{i} \Leftrightarrow \mu_{k}} \\
= & s_{2 j} \sum_{k \neq i}\left(\left(e_{2 j}, w_{i}\right)\left(e_{2 j+1}, w_{k}\right)+\left(e_{2 j}, w_{k}\right)\left(e_{2 j+1}, w_{i}\right)\right) \frac{w_{k}}{\mu_{i} \Leftrightarrow \mu_{k}} \\
= & s_{2 j} \sum_{k \neq i}\left(\mu_{i}\left(z_{2 j}, w_{i}\right)\left(e_{2 j+1}, w_{k}\right)+\mu_{k}\left(z_{2 j}, w_{k}\right)\left(e_{2 j+1}, w_{i}\right)\right) \frac{w_{k}}{\mu_{i} \Leftrightarrow \mu_{k}}, \quad \text { as } S(t) z_{2 j}=e_{2 j}, \\
= & \frac{s_{2 j}}{2} \sum_{k \neq i}\left[\left(\frac{\mu_{i}+\mu_{k}}{\mu_{i} \Leftrightarrow \mu_{k}}\right)\left(z_{2 j}, w_{i}\right)\left(e_{2 j+1}, w_{k}\right)+\left(\frac{\mu_{k}+\mu_{i}}{\mu_{i} \Leftrightarrow \mu_{k}}\right)\left(z_{2 j}, w_{k}\right)\left(e_{2 j+1}, w_{i}\right)\right] w_{k} \\
& \quad+\frac{s_{2 j}}{2} \sum_{k \neq i}\left[\left(z_{2 j}, w_{i}\right)\left(e_{2 j+1}, w_{k}\right) \Leftrightarrow\left(z_{2 j}, w_{k}\right)\left(e_{2 j+1}, w_{i}\right)\right] w_{k} .
\end{aligned}
$$

The second sum is bounded in norm by

$$
\left|\left(z_{2 j}, w_{i}\right)\right|\left\|e_{2 j+1}\right\|_{2}+\left|\left(e_{2 j+1}, w_{i}\right)\right|\left\|z_{2 j}\right\|_{2} \leq \sqrt{2}\left\|z_{2 j}\right\|_{2} \text {, by }(4.9) .
$$

The first sum is bounded in norm by

$$
\begin{gathered}
\max _{k \neq i}\left|\frac{\mu_{i}+\mu_{k}}{\mu_{i} \Leftrightarrow \mu_{k}}\right|\left\{\sum _ { k = 1 } ^ { 2 n } \left[\left(z_{2 j}, w_{i}\right)^{2}\left(e_{2 j+1}, w_{k}\right)^{2}+\left(z_{2 j}, w_{k}\right)^{2}\left(e_{2 j+1}, w_{i}\right)^{2}\right.\right. \\
\left.\left.+2\left(z_{2 j}, w_{i}\right)\left(e_{2 j+1}, w_{i}\right)\left(e_{2 j+1}, w_{k}\right)\left(z_{2 j}, w_{k}\right)\right]\right\}^{1 / 2}
\end{gathered}
$$




$$
\begin{aligned}
& =\max _{k \neq i}\left|\frac{\mu_{i}+\mu_{k}}{\mu_{i} \Leftrightarrow \mu_{k}}\right|\left\{\frac{\left\|z_{2 j}\right\|_{2}^{2}}{2}+\frac{\left\|z_{2 j}\right\|_{2}^{2}}{2}+2 \cdot\left(2^{-1 / 2}\left\|z_{2 j}\right\|_{2}\right) \cdot 2^{-1 / 2} \cdot\left\|z_{2 j}\right\|_{2}\right\}^{1 / 2} \\
& =\sqrt{2}\left(\max _{k \neq i}\left|\frac{\mu_{i}+\mu_{k}}{\mu_{i} \Leftrightarrow \mu_{k}}\right|\right)\left\|z_{2 j}\right\|_{2} .
\end{aligned}
$$

Combining terms $\Gamma$

$$
\left\|\frac{d \omega_{i}(t)}{d t}\right\|_{2} \leq \frac{s_{2 j}\left\|z_{2 j}\right\|_{2}}{\sqrt{2}}\left(1+\max _{k \neq i}\left|\frac{\mu_{i}(t)+\mu_{k}(t)}{\mu_{i}(t) \Leftrightarrow \mu_{k}(t)}\right|\right)
$$

A similar computation shows that the same inequality holds with $\left\|z_{2 j}\right\|_{2}$ replaced by $\left\|z_{2 j+1}\right\|_{2}$. Furthermore note that the proof of $(4.7)_{\ell}$ shows that

$$
1 \Leftrightarrow m_{\ell}^{\prime}<e^{-m_{\ell}} \leq e^{-(1-t) m_{\ell}} \leq \frac{\mu_{i}(t)}{\mu_{i}} \leq e^{(1-t) m_{\ell}} \leq e^{m_{\ell}}=1+m_{\ell}^{\prime}
$$

and henceTarguing as in the proof of Theorem $2.11 \mathrm{\Gamma}$

$$
\left\|\frac{d h_{i}^{ \pm}(t)}{d t}\right\|_{2} \leq \frac{m_{2}}{\sqrt{2}}\left(1+\frac{1+m_{2}^{\prime}}{\text { relgap } i \Leftrightarrow m_{2}^{\prime}}\right),
$$

which yields (4.8) $)_{\ell}$ Tupon integration.

The inequality $(4.8)_{1}$ is proved in the same way. Factors of order $\sqrt{n}$ appearTfor example in estimating the term

$$
\sum_{k \neq i}\left(z_{2 j}, w_{k}\right)^{2} \leq(2 n \Leftrightarrow 1)\left(2^{-1 / 2}\left\|z_{2 j}\right\|_{1}\right.
$$

Remark. The inequality $(4.7)_{\ell}$ was proved in [DK88] by a different method. 


\section{Error Bounds for the Implicit Zero-Shift QR Algorithm}

In this section we will derive error bounds for the quantities computed by $m$ steps of the implicit zero-shift QR algorithm of section 3. We will use the bound on $\|M(i, j)\|$ to be derived in section 9 to bound the round off error propagated from step to step. Our main results will be Lemma $5.11 \Gamma$ which bounds the relative errors in the bidiagonal matrix entries after $m$ zero-shift QR steps Tand Lemma 5.14 Which bounds the absolute error in the computed orthogonal matrix of the $m$ zero-shift $Q R$ steps.

In the error analysis $\Gamma$ we will use the fact that an absolute perturbation $\epsilon$ in $\log x$ is to first order equivalent to a relative perturbation $\epsilon$ in $x$ :

$$
\log x(1+\epsilon)=\log x+\log (1+\epsilon) \approx \log x+\epsilon
$$

Therefore the Jacobian map $M(j, i)$ which propagates absolute perturbations in the logarithms of entries of $B_{i}$ to $B_{j}$ also propagates relative perturbations of entries of $B_{i}$ to $B_{j}$.

As is traditional in numerical analysisTwe will bound quantities of the form $\prod\left(1+\epsilon_{i}\right)$ by instead bounding $s \equiv \sum\left|\epsilon_{i}\right|$. When $s \ll 1$ (the case of interest) $\Gamma$ we then have approximately that $1 \Leftrightarrow s \leq \prod\left(1+\epsilon_{i}\right) \leq 1+s$. If more rigor is desired $\Gamma$ we can wse the fact that for all $s<1$ we have $1 \Leftrightarrow s \leq \prod\left(1+\epsilon_{i}\right) \leq \epsilon^{s}$.

Our model of arithmetic is a variation on the standard one: the floating point result $f l(\cdot)$ of the operation $(\cdot)$ is given by

$$
\begin{aligned}
f l(a \pm b) & =a\left(1+\varepsilon_{1}\right) \pm b\left(1+\varepsilon_{2}\right) \\
f l(a \times b) & =(a \times b)\left(1+\varepsilon_{3}\right) \\
f l(a / b) & =(a / b)\left(1+\varepsilon_{4}\right) \\
f l(\sqrt{a}) & =\sqrt{a}\left(1+\varepsilon_{5}\right)
\end{aligned}
$$

where $\left|\varepsilon_{i}\right| \leq \varepsilon \Gamma$ and $\varepsilon \ll 1$ is the machine precision. This is somewhat more general than the usual model which uses $f l(a \pm b)=(a \pm b)\left(1+\varepsilon_{1}\right)$ and includes machines like the Cray which do not have a guard digit. We do not consider over/underflow; methods for extending error analysis to include underflow are presented in [Dem84].

Our analysis proceeds by five lemmas. Lemma 5.2 [DK88] analyzes the roundoff errors in the subroutine ROT of section 3. Lemma 5.4 uses Lemma 5.2 to bound the errors in the bidiagonal matrix $B$ after one step of the implicit zero-shift QR algorithm. Lemma 5.11 uses Lemma 5.4 and the bound on $\|M(j, i)\|$ of section 9 to bound the errors in the bidiagonal matrix after $m$ steps of the implicit zero-shift QR algorithm. Lemma 5.13 shows that small errors in the sines and cosines computed by one step of the algorithm only cause small errors in the computed orthogonal matrices containing the singular vectors. Finally Lemma 5.14 bounds the absolute error in the computed orthogonal matrices containing the singular vectors after $m$ steps of the algorithm.

Lemma 5.2 Let $\cos \theta, \sin \theta$ and $\rho$ denote the exact outputs of ROT for inputs $f$ and $g$ and exact arithmetic. Let $c s=\left(1+\epsilon_{c s}\right) \cos \theta, s n=\left(1+\epsilon_{s n}\right) \sin \theta$ and $r=\left(1+\epsilon_{r}\right) \rho$ denote the floating point results of ROT applied to the perturbed inputs $\hat{f}=\left(1+\epsilon_{f}\right) f$ and $\hat{g}=\left(1+\epsilon_{g}\right) g$. 
Then to first order we may bound the relative errors $\epsilon_{c s}, \epsilon_{s n}$ and $\epsilon_{r}$ in terms of $\epsilon_{f}, \epsilon_{g}$ and the machine precision $\varepsilon$ as follows:

$$
\begin{aligned}
\left|\epsilon_{c s}\right| & \leq\left(\left|\epsilon_{f}\right|+\left|\epsilon_{g}\right|\right) \sin ^{2} \theta+\frac{21 \varepsilon}{4} \\
\left|\epsilon_{s n}\right| & \leq\left(\left|\epsilon_{f}\right|+\left|\epsilon_{g}\right|\right) \cos ^{2} \theta+\frac{21 \varepsilon}{4} \\
\left|\epsilon_{r}\right| & \leq\left|\epsilon_{f}\right| \cos ^{2} \theta+\left|\epsilon_{g}\right| \sin ^{2} \theta+\frac{13 \varepsilon}{4}
\end{aligned}
$$

Proof. See the proof of Lemma 5 in [DK88]. The slightly different model $f l(a \pm b)=$ $(a \pm b)\left(1+\varepsilon_{1}\right)$ used in [DK88] does not affect the final result because only positive quantities are added in $R O T$; this makes the two models equivalent.

To state the next lemma we need to be able to distinguish the different values the variables in the implicit zero-shift $Q R$ algorithm take on at different times. To this end $\Gamma$ we state the following equivalent algorithm $\mathrm{W}$ where the variables are labeled by the loop counter $i$ :

\section{Labeled Implicit Zero-Shift QR Algorithm:}

$$
\begin{aligned}
& \text { oldcs } s_{1}=1 \\
& f_{1}=a_{1} \\
& g_{1}=b_{1} \\
& \text { for } i=1 \Gamma n \Leftrightarrow 1 \\
& \quad \text { call } R O T\left(f_{i}, g_{i}, c s_{i 1}, s n_{i 1}, r_{i 1}\right) \\
& \quad \quad \text { call } U D P A T E\left(c s_{i 1}, s n_{i 1}, v_{i}, v_{i+1}\right) \\
& \quad \text { if }(i \neq 1) \Gamma b_{i-1}=\text { old } s n_{i} * r_{i 1} \\
& \quad f_{i 1}=\text { oldcs } s_{i} * r_{i 1} \\
& \quad g_{i 1}=a_{i+1} * s n_{i 1} \\
& \quad h_{i}=a_{i+1} * c s_{i 1} \\
& \quad \text { call } R O T\left(f_{i 1}, g_{i 1}, c s_{i 2}, s n_{i 2}, r_{i 2}\right) \\
& \quad \text { call } U P D A T E\left(c s_{i 2}, s n_{i 2}, u_{i}, u_{i+1}\right) \\
& \quad a_{i}=r_{i 2} \\
& \quad f_{i+1}=h_{i} \\
& \quad g_{i+1}=b_{i+1} \\
& \quad \text { old } c s_{i+1}=c s_{i 2} \\
& \quad \text { old } s n_{i+1}=s n_{i 2} \\
& \text { endfor } \\
& b_{n-1}=h_{n-1} * s n_{n-1,2} \\
& a_{n}=h_{n-1} * c s_{n-1,2}
\end{aligned}
$$

Let the $n$ by $n$ bidiagonal matrix $B$ have diagonal entries $a_{i}$ and offdiagonal entries $b_{i}$. Let the matrix $B^{\prime}$ with entries $a_{i}^{\prime}$ and $b_{i}^{\prime}$ be the the result of applying the implicit zero-shift QR algorithm to $B$ once in exact arithmetic $\Gamma$ and let the variables in the labeled implicit zero-shift $\mathrm{QR}$ algorithm above denote the corresponding intermediate values.

Now let $\hat{B}$ be the slightly perturbed matrix with entries $\hat{a}_{i}=a_{i}\left(1+\epsilon_{a_{i}}\right)$ and $\hat{b}_{i}=$ $b_{i}\left(1+\epsilon_{b_{i}}\right)$ Tand let $\hat{B}^{\prime}$ (with entries $\hat{a}_{i}^{\prime}=a_{i}^{\prime}\left(1+\epsilon_{a_{i}^{\prime}}\right)$ and $\hat{b}_{i}^{\prime}=b_{i}^{\prime}\left(1+\epsilon_{b_{i}^{\prime}}\right)$ ) be the result of applying the implicit zero-shift $\mathrm{QR}$ algorithm in floating point arithmetic to $\hat{B}$. Let hatted 
variable denote the corresponding intermediate floating point variables (e.g. $\hat{f}_{i}=f_{i}\left(1+\epsilon_{f_{i}}\right)$ ). We wish to bound the final relative errors $\epsilon_{a_{i}^{\prime}}$ and $\epsilon_{b_{i}^{\prime}}$ in the entries of $\hat{B}^{\prime}$ and the relative errors $\epsilon_{\hat{c} s_{i 1}} \epsilon_{\hat{s i n}_{i 1}} \Gamma \epsilon_{\hat{c_{s}}{ }_{i 2}}$ Tand $\epsilon_{\hat{s} \hat{n}_{i 2}}$ in the sines and cosines in terms of the initial relative errors $\epsilon_{a_{i}}$ and $\epsilon_{b_{i}}$ and the machine precision $\varepsilon$.

Lemma 5.4 In terms of the notation just introduced,

$$
\max _{i}\left(\left|\epsilon_{a_{i}^{\prime}}\right|,\left|\epsilon_{b_{i}^{\prime}}\right|\right) \leq 6(2 n \Leftrightarrow 1) \max _{i}\left(\left|\epsilon_{a_{i}}\right|,\left|\epsilon_{b_{i}}\right|\right)+(47 n \Leftrightarrow 27) \varepsilon
$$

and

$$
\epsilon_{c s, s n} \equiv \max _{i}\left(\left|\epsilon_{\hat{\mathcal{C}} s_{i 1}}\right|,\left|\epsilon_{\hat{s n_{i 1}}}\right|,\left|\epsilon_{\hat{c_{s} s_{i 2}}}\right|,\left|\epsilon_{\hat{s n_{i 2}}}\right|\right) \leq 5(2 n \Leftrightarrow 1) \max _{i}\left(\left|\epsilon_{a_{i}}\right|,\left|\epsilon_{b_{i}}\right|\right)+(41 n \Leftrightarrow 66) \varepsilon
$$

Remark 5.7 Lemma 5.4 is a variation on Lemma 7 in [DK88].

Proof. We begin systematically applying (5.1) and Lemma 5.2 to all the operations in the labeled implicit zero-shift QR algorithm in order to derive a recurrence relation for $\left|\epsilon_{f_{i}}\right|$ and $\left|\epsilon_{\text {oldcsi }}\right|$. Initially

$$
\hat{f}_{1} \equiv f_{1}\left(1+\epsilon_{f_{1}}\right)=a_{1}\left(1+\epsilon_{a_{1}}\right) \quad \text { and } \quad \hat{g}_{1} \equiv g_{1}\left(1+\epsilon_{g_{1}}\right)=b_{1}\left(1+\epsilon_{b_{1}}\right)
$$

so that $\epsilon_{f_{1}}=\epsilon_{a_{1}}$ and $\epsilon_{g_{1}}=\epsilon_{b_{1}}$. At the top of the loop we always have $\hat{g}_{i}=\hat{b}_{i}$ and so $\epsilon_{g_{i}}=\epsilon_{b_{i}}$. After the first call to $R O T$ we have

$$
\begin{aligned}
& \hat{c s_{i 1}} \equiv c s_{i 1}\left(1+\epsilon_{c s_{i 1}}\right) \quad \text { where } \quad\left|\epsilon_{c s_{i 1}}\right| \leq\left(\left|\epsilon_{f_{i}}\right|+\left|\epsilon_{b_{i}}\right|\right) s n_{i 1}^{2}+\frac{21 \varepsilon}{4} \\
& \hat{s}_{i 1} \equiv s n_{i 1}\left(1+\epsilon_{s n_{i 1}}\right) \quad \text { where } \quad\left|\epsilon_{s n_{i 1}}\right| \leq\left(\left|\epsilon_{f_{i}}\right|+\left|\epsilon_{b_{i}}\right|\right) c s_{i 1}^{2}+\frac{21 \varepsilon}{4} \\
& \hat{r}_{i 1} \equiv r_{i 1}\left(1+\epsilon_{r_{i 1}}\right) \quad \text { where } \quad\left|\epsilon_{r_{i 1}}\right| \leq\left|\epsilon_{f_{i}}\right| c s_{i 1}^{2}+\left|\epsilon_{b_{i}}\right| s n_{i 1}^{2}+\frac{13 \varepsilon}{4}
\end{aligned}
$$

NextTwe get

$$
\begin{aligned}
\hat{f}_{i 1} \equiv f_{i 1}\left(1+\epsilon_{f_{i 1}}\right) & \text { where } & \left|\epsilon_{f_{i 1}}\right| \leq\left|\epsilon_{\text {oldcsi }}\right|+\left|\epsilon_{r_{i 1}}\right|+\varepsilon \leq\left|\epsilon_{\text {oldcsi }}\right|+\left|\epsilon_{f_{i}}\right| c s_{i 1}^{2}+\left|\epsilon_{b_{i}}\right| s n_{i 1}^{2}+\frac{17 \varepsilon}{4} \\
\hat{g}_{i 1} \equiv g_{i 1}\left(1+\epsilon_{g_{i 1}}\right) & \text { where } & \left|\epsilon_{g_{i 1}}\right| \leq\left|\epsilon_{a_{i+1}}\right|+\left|\epsilon_{s n_{i 1}}\right|+\varepsilon \leq\left|\epsilon_{a_{i+1}}\right|+\left(\left|\epsilon_{f_{i}}\right|+\left|\epsilon_{b_{i}}\right|\right) c s_{i 1}^{2}+\frac{25 \varepsilon}{4} \\
\hat{h}_{i} \equiv h_{i}\left(1+\epsilon_{h_{i}}\right) & \text { where } & \left|\epsilon_{h_{i}}\right| \leq\left|\epsilon_{a_{i+1}}\right|+\left|\epsilon_{c s_{i 1}}\right|+\varepsilon \leq\left|\epsilon_{a_{i+1}}\right|+\left(\left|\epsilon_{f_{i}}\right|+\left|\epsilon_{b_{i}}\right|\right) s n_{i 1}^{2}+\frac{25 \varepsilon}{4}
\end{aligned}
$$

After the second call to ROT we have

$$
\begin{array}{rrr}
\hat{c} s_{i 2} \equiv c s_{i 2}\left(1+\epsilon_{c s_{i 2}}\right) \quad \text { where }\left|\epsilon_{c s_{i 2}}\right| \leq & \left(\left|\epsilon_{\text {oldcsi }}\right|+\left|\epsilon_{a_{i+1}}\right|+2\left|\epsilon_{f_{i}}\right| c s_{i 1}^{2}+\left|\epsilon_{b_{i}}\right|\right) s n_{i 2}^{2}+\frac{63 \varepsilon}{4} \\
\hat{s n}_{i 2} \equiv s n_{i 2}\left(1+\epsilon_{s n_{i 2}}\right) \quad \text { where }\left|\epsilon_{s n_{i 2}}\right| \leq & \left(\left|\epsilon_{\text {oldcsi }}\right|+\left|\epsilon_{a_{i+1}}\right|+2\left|\epsilon_{f_{i}}\right| c s_{i 1}^{2}+\left|\epsilon_{b_{i}}\right|\right) c s_{i 2}^{2}+\frac{63 \varepsilon}{4} \\
\hat{r}_{i 2} \equiv r_{i 2}\left(1+\epsilon_{r_{i 2}}\right) \quad \text { where }\left|\epsilon_{r_{i 2}}\right| \leq & \left|\epsilon_{o l d c s_{i}}\right| c s_{i 2}^{2}+\left|\epsilon_{a_{i+1}}\right| s n_{i 2}^{2}+\left|\epsilon_{f_{i}}\right| c s_{i 1}^{2} \\
& +\left|\epsilon_{b_{i}}\right|\left(c s_{i 2}^{2} s n_{i 1}^{2}+s n_{i 2}^{2} c s_{i 1}^{2}\right)+\frac{55 \varepsilon}{4}
\end{array}
$$


Since oldcs $s_{i+1}=c s_{i 2}$ and $f_{i+1}=h_{i}$ Twe have $\epsilon_{o l d c s_{i+1}}=\epsilon_{c s_{i 2}}$ and $\epsilon_{f_{i+1}}=\epsilon_{h_{i}}$ Tso $\left[\begin{array}{c}\left|\epsilon_{f_{i+1}}\right| \\ \left|\epsilon_{\text {old } c s_{i+1}}\right|\end{array}\right] \leq\left[\begin{array}{cc}s n_{i 1}^{2} & 0 \\ 2 c s_{i 1}^{2} s n_{i 2}^{2} & s n_{i 2}^{2}\end{array}\right] \cdot\left[\begin{array}{c}\left|\epsilon_{f_{i}}\right| \\ \left|\epsilon_{\text {oldcsi }}\right|\end{array}\right]+\left[\begin{array}{cc}s n_{i 1}^{2} & 1 \\ s n_{i 2}^{2} & s n_{i 2}^{2}\end{array}\right] \cdot\left[\begin{array}{c}\left|\epsilon_{b_{i}}\right| \\ \left|\epsilon_{a_{i+1}}\right|\end{array}\right]+\left[\begin{array}{c}25 \\ 63\end{array}\right] \frac{\varepsilon}{4}$

We may write this as

$$
E_{i+1} \leq A_{i} \cdot E_{i}+F_{i}+G_{i}
$$

where

$$
E_{i}=\left[\begin{array}{c}
\left|\epsilon_{f_{i}}\right| \\
\left|\epsilon_{\text {oldcsi }}\right|
\end{array}\right], F_{i}=\left[\begin{array}{l}
1 \\
1
\end{array}\right]\left(\left|\epsilon_{b_{i}}\right|+\left|\epsilon_{a_{i+1}}\right|\right) \quad \text { and } \quad G_{i}=\left[\begin{array}{c}
25 \\
63
\end{array}\right] \frac{\varepsilon}{4}
$$

This implies

$$
E_{i+1} \leq \sum_{j=1}^{i}\left(A_{i} \cdots A_{j+1}\right)\left(F_{j}+G_{j}\right)+\left(A_{i} \cdots A_{1}\right) E_{1}
$$

Since componentwise

$$
A_{i} \cdots A_{j+1} \leq\left[\begin{array}{cc}
s n_{i 1}^{2} & 0 \\
2 c s_{i 1}^{2} & 1
\end{array}\right] \cdots\left[\begin{array}{cc}
s n_{j+1,1}^{2} & 0 \\
2 c s_{j+1,1}^{2} & 1
\end{array}\right]=\left[\begin{array}{cc}
s n_{i 1}^{2} \cdots s n_{j+1,1}^{2} & 0 \\
2\left(1 \Leftrightarrow s n_{i 1}^{2} \cdots s n_{j+1,1}^{2}\right) & 1
\end{array}\right] \leq\left[\begin{array}{ll}
1 & 0 \\
2 & 1
\end{array}\right]
$$

we finally get that

$$
E_{i+1}=\left[\begin{array}{c}
\left|\epsilon_{f_{i+1}}\right| \\
\mid \epsilon_{\text {oldcs }}+1
\end{array}\right] \leq\left[\begin{array}{l}
1 \\
3
\end{array}\right]\left(\sum_{j=1}^{i}\left|\epsilon_{b_{j}}\right|+\sum_{j=1}^{i+1}\left|\epsilon_{a_{j}}\right|\right)+\left[\begin{array}{c}
25 \\
113
\end{array}\right] \frac{i \varepsilon}{4}
$$

Now we can bound $\left|\epsilon_{a_{i}^{\prime}}\right|$ and $\left|\epsilon_{b_{i}^{\prime}}\right|$. From the algorithm we see for $i \leq n \Leftrightarrow 1$ we have $a_{i}^{\prime}=r_{i 2}$ so that $\epsilon_{a_{i}^{\prime}}=\epsilon_{r_{i 2}}$. Substituting the bounds for $\left|\epsilon_{o l d c s_{i}}\right|$ and $\left|\epsilon_{f_{i}}\right|$ from (5.8) into the bound for $\left|\epsilon_{r_{i 2}}\right|$ and simplifying yields

$$
\left|\epsilon_{a_{i}^{\prime}}\right| \leq 4\left(\sum_{j=1}^{i}\left|\epsilon_{b_{j}}\right|+\sum_{j=1}^{i+1}\left|\epsilon_{a_{j}}\right|\right)+(35 i \Leftrightarrow 20) \varepsilon \quad \text { where } \quad i \leq n \Leftrightarrow 1
$$

We also see from the algorithm that $a_{n}=h_{n-1} * c s_{n-1,2}$ Twhich implies $\left|\epsilon_{a_{n}^{\prime}}\right| \leq \varepsilon+\left|\epsilon_{h_{n-1}}\right|+$ $\left|\epsilon_{c s_{n-1,2}}\right|$. Substituting the bounds for $\left|\epsilon_{o l d c s_{i}}\right|$ and $\left|\epsilon_{f_{i}}\right|$ from (5.8) into the bounds for $\left|\epsilon_{h_{n-1}}\right|$ and $\left|\epsilon_{c s_{n-1,2}}\right|$ Tadding and simplifying $\Gamma$ yields

$$
\left|\epsilon_{a_{n}^{\prime}}\right| \leq 5\left(\sum_{j=1}^{n-1}\left|\epsilon_{b_{j}}\right|+\sum_{j=1}^{n}\left|\epsilon_{a_{j}}\right|\right)+(41 n \Leftrightarrow 58) \varepsilon
$$

Next we see from the algorithm that $b_{i}=$ olds $n_{i+1} * r_{i+1,1}$ for $i \leq n \Leftrightarrow 2$. Since oldsn $n_{i+1}=$ $s n_{i 2}$ Tthis implies $\left|\epsilon_{b_{i}^{\prime}}\right| \leq \varepsilon+\left|\epsilon_{s n_{i 2}}\right|+\left|\epsilon_{r_{i+1}}\right|$. Substituting the bounds for $\left|\epsilon_{\text {oldcs }}\right|$ and $\left|\epsilon_{f_{i}}\right|$ from (5.8) into the bounds for $\left|\epsilon_{s n_{i 2}}\right|$ and $\left|\epsilon_{r_{i+1}}\right| \Gamma$ simplifying and adding yields

$$
\left|\epsilon_{b_{i}^{\prime}}\right| \leq 6\left(\sum_{j=1}^{i+1}\left|\epsilon_{b_{j}}\right|+\sum_{j=1}^{i+1}\left|\epsilon_{a_{j}}\right|\right)+(47 n \Leftrightarrow 27) \varepsilon \quad \text { where } \quad i \leq n \Leftrightarrow 2
$$


We also see from the algorithm that $b_{n-1}=h_{n-1} * s n_{n-1,2} \Gamma$ which implies $\left|\epsilon_{b_{n-1}^{\prime}}\right| \leq \varepsilon+$ $\left|\epsilon_{h_{n-1}}\right|+\left|\epsilon_{s n_{n-1,2}}\right|$. Substituting the bounds for $\left|\epsilon_{o l d c s_{i}}\right|$ and $\left|\epsilon_{f_{i}}\right|$ from (5.8) into the bounds for $\left|\epsilon_{h_{n-1}}\right|$ and $\left|\epsilon_{s n_{n-1,2}}\right|$ Tsimplifying and adding yields

$$
\left|\epsilon_{b_{n-1}^{\prime}}\right| \leq 5\left(\sum_{j=1}^{n-1}\left|\epsilon_{b_{j}}\right|+\sum_{j=1}^{n}\left|\epsilon_{a_{j}}\right|\right)+(41 n \Leftrightarrow 58) \varepsilon
$$

Combining the last four displayed inequalities yields claim (5.5) of the lemma.

Next we bound the relative errors in the computed sines and cosines $\epsilon_{s n_{i 1}} \Gamma \epsilon_{s n_{i 2}} \Gamma \epsilon_{C s_{i 1}}$ and $\epsilon_{c s_{i 2}}$. Substituting the bounds for $\left|\epsilon_{o l d c s_{i}}\right|$ and $\left|\epsilon_{f_{i}}\right|$ from (5.8) into the earlier bounds on the $\left|\epsilon_{s n_{i 1}}\right| \Gamma\left|\epsilon_{s n_{i 2}}\right| \Gamma\left|\epsilon_{c s_{i 1}}\right|$ and $\left|\epsilon_{c s_{i 2}}\right|$ and simplifying yields

$$
\begin{gathered}
\left|\epsilon_{s n_{i 1}}\right| \leq \sum_{j=1}^{i}\left|\epsilon_{b_{j}}\right|+\sum_{j=1}^{i}\left|\epsilon_{a_{j}}\right|+(7 i \Leftrightarrow 1) \varepsilon \\
\left|\epsilon_{s n_{i 2}}\right| \leq 5\left(\sum_{j=1}^{i}\left|\epsilon_{b_{j}}\right|+\sum_{j=1}^{i+1}\left|\epsilon_{a_{j}}\right|\right)+(41 i \Leftrightarrow 25) \varepsilon \\
\left|\epsilon_{c s_{i 1}}\right| \leq \sum_{j=1}^{i}\left|\epsilon_{b_{j}}\right|+\sum_{j=1}^{i}\left|\epsilon_{a_{j}}\right|+(7 i \Leftrightarrow 1) \varepsilon \\
\left|\epsilon_{c s_{i 2}}\right| \leq 5\left(\sum_{j=1}^{i}\left|\epsilon_{b_{j}}\right|+\sum_{j=1}^{i+1}\left|\epsilon_{a_{j}}\right|\right)+(41 i \Leftrightarrow 25) \varepsilon
\end{gathered}
$$

Combining the last four inequalities yields

$$
\epsilon_{c s, s n} \equiv \max _{i}\left(\left|\epsilon_{s n_{i 1}}\right|,\left|\epsilon_{s n_{i 2}}\right|,\left|\epsilon_{c s_{i 1}}\right|,\left|\epsilon_{c s_{i 2}}\right|\right) \leq 5\left(\sum_{j=1}^{n-1}\left|\epsilon_{b_{j}}\right|+\sum_{j=1}^{n}\left|\epsilon_{a_{j}}\right|\right)+(41 n \Leftrightarrow 66) \varepsilon
$$

implying (5.6).

Now we bound the relative error in the computed bidiagonal matrix after $m$ steps of the implicit zero-shift QR algorithm. For reasons which will become clear shortly denote our initial bidiagonal matrix by $B^{(00)}$ Tthe matrix after $m$ steps of the algorithm in exact arithmetic by $B^{(m 0)}$ Tand the matrix after $m$ steps of the algorithm in floating point arithmetic by $B^{(\mathrm{mm})}$. We wish to bound the maximum componentwise relative difference between $B^{(m 0)}$ and $B^{(m m)}$ Twhich we measure by

$$
\operatorname{reldif}\left(B^{(m 0)}, B^{(m m)}\right) \equiv \max _{i j}\left|\log \frac{B_{i j}^{(m 0)}}{B_{i j}^{(m 0)}}\right|
$$

the subscripts $i, j$ varying over the bidiagonal entries.

The proof will use the matrix $M\left(m_{1}, m_{2}\right)$ Twhich is the Jacobian matrix of the transformation induced by the algorithm in going from the $m_{1}$-st to the $m_{2}$-nd bidiagonal matrix but in the variables $\log a_{i}$ and $\log b_{i}$. Because small absolute perturbations of the logarithms $\log a_{i}+\epsilon$ are the same as small relative perturbations of the matrix entries $\log a_{i}(1+\epsilon) \Gamma$ 
$M\left(m_{1}, m_{2}\right)$ measures how relative errors in the $m_{1}$-st bidiagonal matrix are propagated to the $m_{2}-$ nd.

$M\left(m_{1}, m_{2}\right)$ will be analyzed in detail in section 9 Tbut we need just one result (Theorem $9.23)$ from that section:

$$
\left\|M\left(m_{1}, m_{2}\right)\right\|_{\infty} \leq(8 n \Leftrightarrow 4)\left(m_{2} \Leftrightarrow m_{1}\right)+O(1)
$$

where $n$ is the dimension of the bidiagonal matrix. The numerical simulations in section 11 show that the $O(1)$ can indeed be replaced by zero.

Since the bound (5.9) does not depend on the bidiagonal matrix global error bounds: if $B_{1}$ and $B_{2}$ are two bidiagonal matrices $\Gamma$ and $B_{1}(m)$ and $B_{2}(m)$ are the matrices after $m$ applications of the algorithm in exact arithmetic $\Gamma$ then

$$
\text { reldif }\left(B_{1}(m), B_{2}(m)\right) \leq((8 n \Leftrightarrow 4) m+O(1)) \cdot \operatorname{reldif}\left(B_{1}, B_{2}\right)
$$

Lemma 5.11 In terms of the above notation

$$
\text { reldif }\left(B^{(m 0)}, B^{(m m)}\right) \leq 188 n^{2} m^{2} \varepsilon+O(n m \varepsilon)
$$

Remark 5.12 In practice $\Gamma$ as illustrated by the numerical experiments of section $11 \Gamma$ the $O(n m \varepsilon)$ term may be replaced by 0 . By assuming that the angles encountered in the course of the algorithm are bounded away from $\pi / 2$ (which is reasonable 5 since they approach zero in the limit) $\Gamma$ the $n^{2}$ dependence may be replaced by something proportional to $n$ (see Lemma 8 in [DK88]).

Proof. Let $B^{(k k)}$ denote the computed bidiagonal matrix after $k$ steps of the algorithm in floating point arithmetic. Starting from $B^{(k k)} \Gamma$ consider the sequence $B^{(k+1, k)} \Gamma B^{(k+2, k)} \Gamma$ $\ldots \Gamma B^{(m k)}$ which would be computed by applying the algorithm in exact arithmetic to $B^{(k k)}$. We will bound reldif $\left(B^{(m 0)}, B^{(m m)}\right)$ by

$$
\operatorname{reldif}\left(B^{(m 0)}, B^{(m m)}\right) \leq \sum_{k=1}^{m} \operatorname{reldif}\left(B^{(m k)}, B^{(m, k-1)}\right)
$$

Now $B^{(m k)}$ and $B^{(m, k-1)}$ arise from applying the algorithm in exact arithmetic to $B^{(k k)}$ and $B^{(k, k-1)}$ Trespectively. ThereforeTby $(5.10)$ the relative difference between them will be bounded by

$$
\text { reldif }\left(B^{(m k)}, B^{(m, k-1)}\right) \leq((8 n \Leftrightarrow 4)(m \Leftrightarrow k)+O(1)) \operatorname{reldif}\left(B^{(k k)}, B^{(k, k-1)}\right)
$$

But $B^{(k k)}$ is obtained from $B^{(k-1, k-1)}$ by one step of the algorithm in floating point arithmetic $\Gamma$ and $B^{(k, k-1)}$ is obtained from $B^{(k-1, k-1)}$ by one step of the algorithm in exact arithmetic. ThereforeTreldif $\left(B^{(k k)}, B^{(k, k-1)}\right)$ is bounded by (5.5) in Lemma 5.4:

$$
\text { reldif }\left(B^{(k k)}, B^{(k, k-1)}\right) \leq(47 n \Leftrightarrow 27) \varepsilon
$$

Combining the last three displayed equations yields the claimed result.

We note that the method of proof is analogous to the way error bounds are derived for computed solutions of initial value problems for differential equations: The truncation error 
at each step is analogous to our one step error bounded in Lemma 5.4. Then the differential equation being solved is used to propagate the error bound for the truncation error forward; this is exactly what we are doing.

The next lemma shows that if the maximum error $\epsilon_{c s, s n}$ in the computed sines and cosines is small so will be the error in the singular vectors computed by UPDATE:

Lemma 5.13 Let $V$ be an $n$ by $n$ orthogonal matrix, and $V^{\prime}$ the updated orthogonal matrix obtained by running one step of the implicit zero-shift $Q R$ algorithm in exact arithmetic. Let $\delta V$ be a perturbation of $V$, and let $V^{\prime}+\delta V^{\prime}$ be the matrix obtained by running the algorithm in floating point on $V+\delta V$, where we assume the relative errors in the computed sines and cosines are bounded by $\epsilon_{c s, s n}$. Then to first order in $\epsilon_{c s, s n}$, $\varepsilon$, and $\|\delta V\|_{2}$

$$
\left\|\delta V^{\prime}\right\|_{2} \leq 2^{3 / 2}(n \Leftrightarrow 1) \varepsilon+2^{1 / 2}(n \Leftrightarrow 1) \epsilon_{c s, s n}+\|\delta V\|_{2}
$$

Proof. It suffices to analyze the errors from one call to UPDATE. Let $c s$ and $s n$ be the true values of the cosine and sineTand $c s\left(1+\epsilon_{c s}\right)$ and $s n\left(1+\epsilon_{s n}\right)$ the perturbed values. Let $v_{1}$ and $v_{2}$ denote the two columns of $V$ being modified. Then their true new values are

$$
\left[v_{1}^{\prime}, v_{2}^{\prime}\right]=\left[v_{1}, v_{2}\right] \cdot\left[\begin{array}{cc}
c s & \Leftrightarrow s n \\
s n & c s
\end{array}\right]
$$

Let $\left[\delta v_{1}, \delta v_{2}\right]$ be the perturbation of $\left[v_{1}, v_{2}\right]$ due to all previous contributions. Then the $j$-th components of the new perturbation after computing can be written (to first order in $\epsilon_{c s, s n} \Gamma$ $\varepsilon$ and $\left.\|\delta V\|_{2}\right)$ as

$\left[\delta v_{1 j}^{\prime}, \delta v_{2 j}^{\prime}\right]=\left[v_{1 j}, v_{2 j}\right] \cdot\left[\begin{array}{cc}c s\left(\epsilon_{c s}+\varepsilon_{1}+\varepsilon_{2}\right) & \Leftrightarrow s n\left(\epsilon_{s n}+\varepsilon_{3}+\varepsilon_{4}\right) \\ s n\left(\epsilon_{s n}+\varepsilon_{5}+\varepsilon_{6}\right) & c s\left(\epsilon_{c s}+\varepsilon_{7}+\varepsilon_{8}\right)\end{array}\right]+\left[\delta v_{1 j}, \delta v_{2 j}\right] \cdot\left[\begin{array}{cc}c s & \Leftrightarrow s n \\ s n & c s\end{array}\right]$

where $\left|\varepsilon_{i}\right| \leq \varepsilon$. Thus

$$
\left\|\left[\delta v_{1}^{\prime}, \delta v_{2}^{\prime}\right]\right\|_{2} \leq 2^{1 / 2}\left(\epsilon_{c s, s n}+2 \varepsilon\right)+\left\|\left[\delta v_{1}, \delta v_{2}\right]\right\|_{2}
$$

and applying this bound $n \Leftrightarrow 1$ times (for each call to $U P D A T E$ ) we get the result claimed in the lemma.

Lemma 5.14 Let $B$ be an $n$ by $n$ bidiagonal matrix, and let $V$ and $U$ be the orthogonal matrices obtained by running the implicit zero-shift $Q R$ algorithm $m$ times in exact arithmetic. In other words, set $V$ and $U$ to $n$ by $n$ identity matrices initially, and let them be modified by the calls to UPDATE in the algorithm. Now let $\hat{V}$ and $\hat{U}$ be the floating point matrices obtained by running the algorithm in arithmetic of precision $\varepsilon$. Then to first order in $\varepsilon$ we have

$$
\max \left(\|\hat{V} \Leftrightarrow V\|_{2},\|\hat{U} \Leftrightarrow U\|_{2}\right) \leq 947 n^{4}\left(m^{3}+m\right) \varepsilon+O\left(\varepsilon n^{3} m^{2}\right)
$$

Remark 5.16 In the numerical experiments of section 11 the $O\left(\varepsilon n^{3} m^{2}\right)$ term above may be replaced by 0 . By assuming that the angles encountered in the course of the algorithm are bounded away from $\pi / 2$ (which is reasonable $\Gamma$ since they approach zero in the limit) $\Gamma$ the $n^{4}$ in the error bound may be replaced by something proportional to $n^{2}$ (see Lemma 8 in [DK88]). 
Proof. By Lemma 5.13 Tafter each application of the algorithm the error in $V$ increases by

$$
\sqrt{2}(n \Leftrightarrow 1)\left(\epsilon_{c s, s n}+2 \epsilon\right)
$$

By Lemma 5.4 Tat the $k$-th stage $\epsilon_{c s, s n}$ is bounded by

$$
\left|\epsilon_{c s, s n}\right| \leq 5(2 n \Leftrightarrow 1) \operatorname{reldif}\left(B^{(k k)}, B^{(k 0)}\right)+(41 n \Leftrightarrow 66) \varepsilon
$$

By Lemma 5.11 Treldif $\left(B^{(k k)}, B^{(k 0)}\right)$ is bounded by

$$
\text { reldif }\left(B^{(k k)}, B^{(k 0)}\right) \leq \varepsilon\left(188 n^{2} \Leftrightarrow 282 n+54\right)\left(k^{2} \Leftrightarrow k+1\right)+O(n k \varepsilon)
$$

Combining the last three displayed expressions yields

$$
\|V \Leftrightarrow \hat{V}\|_{2} \leq \sum_{k=1}^{m} \sqrt{2}(n \Leftrightarrow 1) \cdot\left(5(2 n \Leftrightarrow 1)\left[\varepsilon\left(188 n^{2} \Leftrightarrow 282 n+54\right)\left(k^{2} \Leftrightarrow k+1\right)+O(n k \varepsilon)\right]+(41 n \Leftrightarrow 66) \varepsilon+2 \varepsilon\right)
$$

which $\Gamma$ when simplified $\Gamma$ yields the desired result. 


\section{Global Error Bounds on the Computed Singular Vectors}

In this section we present the main practical contribution of the paper: an error bound for singular vectors computed by the overall bidiagonal SVD algorithm:

Theorem 6.1 The error in the computed the i-th left and right singular vectors is

$$
p(n, m) \text { tol/relgap }
$$

where tol is the desired relative precision input to the algorithm, $p(n, m)$ is a low degree polynomial in the matrix dimension $n$ and number of $Q R$ steps $m$, and the relative gap relgap $p_{i}$ was defined in 1.7 .

Remark. If we were to apply straightforward error estimates without using the machinery of this paperTthe resulting error bounds would grow exponentially in $n$ and $m$ instead of polynomially.

PROOF. To perform the error analysis we need to associate a tree with the execution of the algorithm. Nodes of the tree will correspond to unreduced submatrices $B_{i}$ upon which the algorithm operates. The root node corresponds to the original matrix and the leaf nodes correspond to 1 by 1 and 2 by 2 submatrices where the algorithm has converged (recall that 2 by 2 matrices are handled specially). A directed edge from node $B_{i}$ to node $B_{j}$ will mean that $B_{j}$ is obtained from $B_{i}$ by performing some step of the algorithm. There

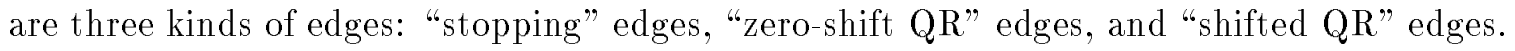
Stopping edges correspond to the stopping criterion deciding to set one or more offdiagonal entries of $B_{i}$ to zero. In this case $B_{j}$ is a submatrix of $B_{i}$. If the stopping criterion sets $p$ offdiagonal entries of $B_{i}$ to zero at the same time $\Gamma B_{i}$ will have $p+1$ child nodes $\Gamma$ one for each resulting submatrix. A zero-shift $Q R$ edge corresponds to one or more applications of the zero-shift QR algorithm. A zero-shift QR edge connecting $B_{i}$ to $B_{j}$ represents all the zero-shift QR steps applied to $B_{i}$ before the stopping criterion is satisfied. Finally if the sequence of QR steps by which $B_{j}$ is obtained from $B_{i}$ includes at least one shifted $\mathrm{QR}$ step $\Gamma$ then $B_{i}$ is connected to $B_{j}$ by a shifted QR edge. Normally a shifted QR edge will represent only shifted QR steps. HoweverTour estimates $\underline{\sigma}\left(B_{i}\right)$ and $\bar{\sigma}\left(B_{i}\right)$ of the smallest and largest singular values of $B_{i}$ Twhich are used to choose between shifted QR and zero-shift QRTare not perfect $\Gamma$ so there is a chance the algorithm could apply both kinds of QR steps to the same submatrix. As we will see $\mathrm{Tthis}$ does not impact the error analysis.

ThusTany path from the root of the tree to a leaf node starts with a QR edge of either typeTand then alternates between stopping edges and QR edges until finally hitting a leaf node at the end of a stopping edge. A node can have at most one entering edge (stopping or QR) Tand either one exiting edge (which must be a QR edge) or more than one exiting edge (which must be stopping edges).

The proof of the theorem proceeds by induction from the leaves of the tree toward the root. We will show that if the computed singular vectors of all the children of a parent node satisfy the error bound $O\left(\right.$ tol $\left._{\text {relga }} \mathrm{p}_{k}\right) \Gamma$ then so do the computed singular vectors of the parent node. First we explain the induction without computing detailed error estimates and then we include the error estimates. In the proof the notation $O(\cdot)$ will absorb all dependence on dimension and number of QR steps. 
First consider the leaf nodes which are all 1 by 1 and 2 by 2 . There is nothing to prove for the 1 by 1 nodes $\Gamma$ and for 2 by 2 nodes the special subroutine discussed in section 3 computes the singular vectors with the desired error bounds.

Now Suppose $B_{i}$ is the first node on the path from the root whose exiting edge is a shifted QR edge. In other wordsTonly zero-shifted QR steps have been applied to $B_{i}$ so far. Starting with $B_{i}$ Tthe algorithm essentially reverts to the standard shifted SVD algorithm $\Gamma$ which is backward stable in the usual normwise sense and so computes the $k$-th singular vectors of $B_{i}$ with an error bound $O\left(\right.$ tol $\left./ a b s g a p_{k}\right) \Gamma$ where absgap $_{k}=\min _{l}\left|\sigma_{k}\left(B_{i}\right) \Leftrightarrow \sigma_{l}\left(B_{i}\right)\right| / \sigma_{1}\left(B_{i}\right)$. From the algorithm of section 3 Wwe see that shifted QR is used only when $\underline{\sigma}\left(B_{i}\right) / \bar{\sigma}\left(B_{i}\right)>$ $n^{-1} \max (\varepsilon /$ tol, .01$)>.01 / n$ Ti.e. only when the smallest singular value of $B_{i}$ is not more than about .01/ $n$ times smaller than the largest singular value. This implies that the relative gap relgap $k=\min _{l}\left|\sigma_{k}\left(B_{i}\right) \Leftrightarrow \sigma_{l}\left(B_{i}\right)\right| /\left|\sigma_{k}\left(B_{i}\right)+\sigma_{l}\left(B_{i}\right)\right|$ cannot be more than about $200 n$

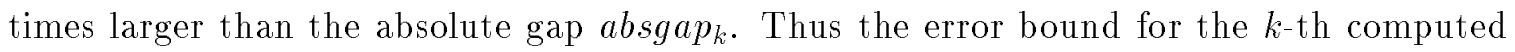
singular vectors of $B_{i}$ are still $O\left(\right.$ tol/relga $\left.p_{k}\right)$ as desired.

(This is where we use the modification of the original algorithm from [DK88]. If the threshold for using shifted QR had been $n^{-1} \varepsilon /$ tol instead of $n^{-1} \max (\varepsilon /$ tol, .01) Tthe error bound would have been $O\left(\operatorname{tol}^{2} /\left(\varepsilon \cdot\right.\right.$ relgap $\left.\left._{k}\right)\right)$ instead of $O\left(\right.$ tol $\left._{\text {relgap }}\right)$. For tol just slightly larger than $\varepsilon$ there is no difference $\Gamma$ but for tol approaching $\varepsilon^{1 / 2}$ the bound $O\left(\operatorname{tol}^{2} /(\varepsilon\right.$. relgap $\left.p_{k}\right)$ ) is significantly weaker than $O($ tol/relgap $k)$.)

At this point in the induction we have shown that the error bounds for computed singular vectors are $O\left(\varepsilon /\right.$ relgap $\left._{k}\right)$ for all nodes which only have zero-shift $\mathrm{QR}$ edges and stopping edges between them and the root. First consider stopping edges. Suppose $B_{i}$ is the parent node from which the stopping edges issue. By Theorem 4.1T the stopping criterion only changes the singular vectors by $O\left(\right.$ tol $\left._{\text {relgap }}\right)$ Twhere relgap $p_{k}$ is measured with respect to the singular values of $B_{i}$ only. This relgap $p_{k}$ may be larger than the relgap $p_{k}$ measured with respect to the entire matrix (since $B_{i}$ contains only a subset of the spectrum of the original matrix $) \Gamma$ but this only improves the error bound $O\left(\right.$ tol $\left._{\text {relgap }}\right)$. This lets us moves the induction toward the root along stopping edges.

Finally consider a zero-shift $\mathrm{QR}$ edge from $B_{i}$ to $B_{j}$. Let $\operatorname{true}\left(B_{j}\right)$ denote the matrix that would have been computed from $B_{i}$ in exact arithmetic $\Gamma$ and $U$ and $V$ the orthogonal matrices that transform $\operatorname{true}\left(B_{j}\right)$ to $B_{i}: U^{T} \cdot \operatorname{true}\left(B_{j}\right) \cdot V=B_{i}$. We will consider right singular vectors only; the proof for left singular vectors is identical. Let the notation true_vector $_{k}(B)$ denote the true $k$-th right singular vector of the matrix $B \Gamma$ and let comp_vector $_{k}(B)$ denote the computed $k$-th right singular vector of $B$. We want to show that ${\| \text { true_vector }_{k}\left(B_{i}\right) \Leftrightarrow \text { comp_vector }_{k}\left(B_{i}\right) \|_{2}=O\left(\text { tol }_{\text {relgap }}\right) \text {. Note that true_vector }}_{k}\left(B_{i}\right)=$

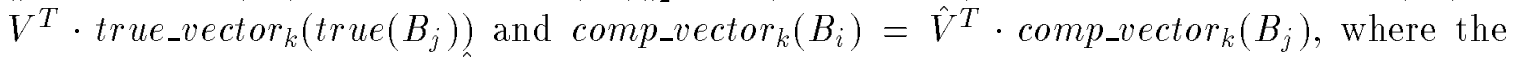
difference between $V$ and $\hat{V}$ is bounded in Lemma 5.14. Consider the following triangle inequality:

$$
\begin{aligned}
\| \text { true_vector }_{k}\left(B_{i}\right) \Leftrightarrow \text { comp_vector }_{k}\left(B_{i}\right) \|_{2} \\
=\| V^{T} \cdot \text { true_vector }_{k}\left(\operatorname{true}\left(B_{j}\right)\right) \Leftrightarrow \hat{V}^{T} \cdot \text { comp_vector }_{k}\left(B_{j}\right) \|_{2} \\
\leq \| V^{T} \cdot \text { true_vector }_{k}\left(\operatorname{true}\left(B_{j}\right)\right) \Leftrightarrow \hat{V}^{T} \cdot \text { true_vector }_{k}\left(\operatorname{true}\left(B_{j}\right)\right) \|_{2}+ \\
\quad \| \hat{V}^{T} \cdot \text { true_vector }_{k}\left(\operatorname{true}\left(B_{j}\right)\right) \Leftrightarrow \hat{V}^{T} \cdot \text { true_vector }_{k}\left(B_{j}\right) \|_{2}+
\end{aligned}
$$




$$
\begin{aligned}
& \| \hat{V}^{T} \cdot \text { true_vector }_{k}\left(B_{j}\right) \Leftrightarrow \hat{V}^{T} \cdot \text { comp_vector }_{k}\left(B_{j}\right) \|_{2} \\
\equiv & I_{1}+I_{2}+I_{3}
\end{aligned}
$$

By Lemma $5.14 \Gamma I_{1}=O(\varepsilon)$. By Theorem 2.12 and Lemma $5.11 \Gamma I_{2}=O\left(\varepsilon /\right.$ relgap $\left._{k}\right)$. By the induction hypothesis $I_{3}=O\left(\right.$ tol $/$ relgap $\left._{k}\right)$. Thus $\Gamma$ the sum $I_{1}+I_{2}+I_{3}=O\left(\right.$ tol $\left._{\text {relgap }}\right)$ as desired. This completes the induction.

Now we consider more rigorous bounds. Explicit formulas for such bounds would be quite complicated and pessimistic and shed little new light on the problem. However Tit is illuminating to use the tree to explain how errors accumulate. We use the fact that if $V_{1}$ and $V_{2}$ are orthogonal $\Gamma$ and $\delta V_{1}$ and $\delta V_{2}$ are small perturbations $\Gamma$ then to first order $\left\|V_{1} V_{2} \Leftrightarrow\left(V_{1}+\delta V_{1}\right)\left(V_{2}+\delta V_{2}\right)\right\|_{2} \leq\left\|\delta V_{1}\right\|_{2}+\left\|\delta V_{2}\right\|_{2}$. This means that to first order perturbation bounds simply add as we proceed up the tree. Thus Tevery time we move along an edge $\Gamma$ we add the error contributed by that edge. For leaf nodes which correspond to 2 by 2 submatricesTwe use error bounds for the special subroutine discussed in section 3 . For stopping edges we used the bounds of Theorem 4.1. For nodes whose exiting edge is the first shifted QR edge we can use the error bounds for the conventional SVD algorithm [GK65]. For zero-shift QR edges we use the analysis of the last paragraph. Only the edges connecting a leaf to the root contribute to the error for the singular vector corresponding to the leaf. 


\section{Flows and the SVD Algorithm}

For a matrix $A$ Tlet $A_{-}$denote its strictly lower triangular part and set $\pi_{0}(A)=A_{-} \Leftrightarrow A_{-}^{T}$. We will consider flows on invertible real matrices $A$ of the form

$$
\frac{d A}{d t}=A\left(\pi_{0}\left(F\left(A^{T} A\right)\right)\right) \Leftrightarrow\left(\pi_{0}\left(F\left(A A^{T}\right)\right)\right) A, \quad A(t=0)=A_{0}
$$

where $F$ is a smooth Treal-valued function on $(0, \infty)$.

Such flows were first considered in the singular value context by M. Chu [Chu] $\mathrm{Cwho}$ analyzed the Toda case $F(x)=x$. For SVD we will set $F(x)=\log x \Gamma$ but initially for reasons of general interest and to suggest additional possibilities $\Gamma$ we will consider general F.

Convention. By the QR factorization of a reall invertible matrix $X$ T we mean $X=$ $Q R \Gamma$ where $Q$ is orthogonal and $R$ is upper triangular with positive diagonal entries (see [GVL83]).

Theorem 7.2 Equation (7.1) has a unique, global solution $A(t)$ which preserves the singular values of $A(t)$. The flow can be solved explicitly, as follows. Let

$$
\begin{aligned}
& e^{t F\left(A_{0}^{T} A_{0}\right)}=Q_{1}(t) R_{1}(t) \\
& e^{t F\left(A_{0} A_{0}^{T}\right)}=Q_{2}(t) R_{2}(t)
\end{aligned}
$$

be the QR-factorizations of $e^{t F\left(A_{0}^{T} A_{0}\right)}$ and $e^{t F\left(A_{0} A_{0}^{T}\right)}$ respectively. Then

$$
A(t)=Q_{2}^{T}(t) A_{0} Q_{1}(t)
$$

Finally, (7.1) preserves bidiagonality i.e. if $A_{0}$ is bidiagonal, then $A(t)$ is bidiagonal for all $t>0$. Moreover the signs of its nonzero entries are preserved.

Proof. Differentiation of (7.3) and (7.4) Tgive

$\frac{d}{d t} Q_{1}=Q_{1} \pi_{0}\left(Q_{1}^{T} F\left(A_{0}^{T} A_{0}\right) Q_{1}\right)=Q_{1} \pi_{0}\left(F\left(\left(Q_{2}^{T} A_{0} Q_{1}\right)^{T}\left(Q_{2}^{T} A_{0} Q_{1}\right)\right)\right)=Q_{1} \pi_{0}\left(F\left(A(t)^{T} A(t)\right)\right)$

and

$\frac{d}{d t} Q_{2}=Q_{2} \pi_{0}\left(Q_{2}^{T} F\left(A_{0} A_{0}^{T}\right) Q_{2}\right)=Q_{2} \pi_{0}\left(F\left(\left(Q_{2}^{T} A_{0} Q_{1}\right)\left(Q_{2}^{T} A_{0} Q_{1}\right)^{T}\right)\right)=Q_{2} \pi_{0}\left(F\left(A(t) A(t)^{T}\right)\right)$.

Thus

$$
\begin{aligned}
\frac{d}{d t} A(t) & =\left[\Leftrightarrow \pi_{0}\left(F\left(A(t) A(t)^{T}\right)\right) Q_{2}^{T}\right] A_{0} Q_{1}+Q_{2}^{T} A_{0}\left[Q_{1} \pi_{0}\left(F\left(A(t)^{T} A(t)\right)\right)\right] \\
& =A(t) \pi_{0}\left(F\left(A(t)^{T} A(t)\right)\right) \Leftrightarrow \pi_{0}\left(F\left(A(t) A(t)^{T}\right)\right) A(t),
\end{aligned}
$$

which is equation (7.1). From $(7.3) \Gamma(7.4) \Gamma(7.5) \Gamma$

$$
\begin{aligned}
A(t) & =\left(R_{2}(t) e^{-t F\left(A_{0} A_{0}^{T}\right)}\right) A_{0}\left(e^{t F\left(A_{0}^{T} A_{0}\right)}\left(R_{1}(t)\right)^{-1}\right) \\
& =R_{2}(t) A_{0}\left(R_{1}(t)\right)^{-1}
\end{aligned}
$$


from which we learn that (7.1) preserves upper triangularity. On the other hand $\Gamma$ we also have

$$
\begin{aligned}
A^{T}(t) A(t) & =Q_{1}(t)^{T} A_{0}^{T} A_{0} Q_{1}(t) \\
& =R_{1}(t) e^{-t F\left(A_{0}^{T} A_{0}\right)} A_{0}^{T} A_{0} e^{t F\left(A_{0}^{T} A_{0}\right)}\left(R_{1}(t)\right)^{-1} \\
& =R_{1}(t) A_{0}^{T} A_{0}\left(R_{1}(t)\right)^{-1}
\end{aligned}
$$

so that $A^{T}(t) A(t)$ is upper HessenbergTand hence tridiagonalГby symmetry. It follows that $A(t)$ is bidiagonal. FurthermoreTit follows from (7.6) and (7.7) that

$$
\operatorname{sgn} A_{i i}(t)=\operatorname{sgn}\left(A_{0}\right)_{i i}, \quad \operatorname{sgn} A_{i i+1}(t)=\operatorname{sgn}\left(A_{0}\right)_{i i+1} .
$$

In particular $\Gamma$ if $a_{p}$ and $b_{q}$ are positive initially they are positive for all time.

Finally the preservation of singular values is immediate from (7.5) Tand this proves the theorem.

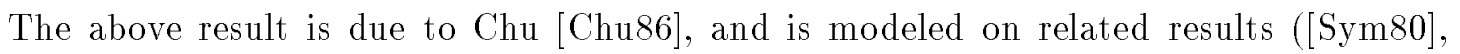
[Sym82]) and Deift-Nanda-Tomei [DNT83] $\mathrm{f}$ for the symmetric eigenvalue problem. The relationship between the singular value flow (7.1) and Toda-type eigenvalue flows ([Sym80]T

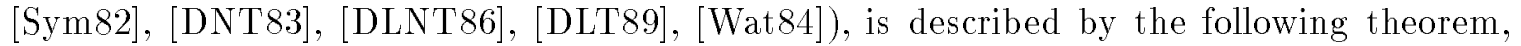
whose proof is immediate.

Theorem 7.9 Under the map

$$
A \mapsto T(A)=A^{T} A
$$

equation (7.1) is transformed into

$$
\frac{d T}{d t}=\left[T, \pi_{0}(F(T))\right]
$$

Remark 7.12 The perfect shuffle $A \mapsto\left(\begin{array}{ll}0 & A^{T} \\ A & 0\end{array}\right) \mapsto S(A)$ of Section $2 \Gamma$ transforms the singular value problem for $A$ into an eigenvalue problem for $S$. One might ask what happens to (7.1) under the map $A \mapsto S$. One finds that (7.1) is transformed into the Toda-type isospectral deformation

$$
\frac{d S}{d t}=\left[S, \pi_{0}\left(F\left(S^{2}\right)\right)\right]
$$

Of particular interest is the case $F(x)=x$. Here (7.11) becomes the Toda flow $\Gamma \frac{d T}{d t}=$ $\left[T, \pi_{0}(T)\right]$ Cbut $(7.13)$ reduces to $\frac{d S}{d t}=\left[S, \pi_{0}\left(S^{2}\right)\right]$; in the case where $A$ is bidiagonal and $S$ takes the form (2.2) Tthis is the so-called Kac-van Moerbeke lattice [KvM75].

The flow that is directly related to the SVD algorithm corresponds to the choice $F(x)=$ $\log x$. To see this note that (7.11) becomes

$$
\frac{d T}{d t}=\left[T, \pi_{0}(\log T)\right], \quad T(0) \equiv A_{0}^{T} A_{0}
$$


whose solution is given by (7.7) $\Gamma$

$$
T(t)=Q_{1}^{T}(t) T(0) Q_{1}(t) .
$$

But from (7.3) Г

$$
T(0)=e^{\log T(0)}=Q_{1}(1) R_{1}(1),
$$

and so

$$
T(1)=R_{1}(1) Q_{1}(1)
$$

Thus

$$
A^{T}(0) A(0)=T(0) \mapsto T(1)=A(1)^{T} A(1)
$$

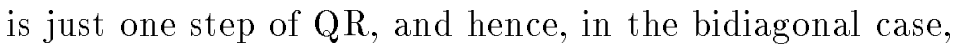

$$
A(0) \mapsto A(1)
$$

is one step of SVD.

In summary we have proven the following basic result.

Theorem 7.14 Let $A_{0}$ be bidiagonal and let $F(x)=\log x$. Then the integer time evaluation of the solution $A(t)$ of (7.1) gives precisely the iterates of the $S V D$ algorithm, $A_{0}, A_{1}, \ldots, A_{k}, \ldots$ Thus

$$
A(k)=A_{k}, \quad k=0,1,2, \ldots .
$$

We will call the flow induced by (7.1) in the case $F(x)=\log x$ Tthe $S V D$ flow.

In the classical case of the Toda flow $\Gamma$ where $T$ is tridiagonal and $F(x)=x \Gamma$ Moser [Mos75] proved the remarkable result that the solution $T(t)$ of (7.11) converges to a diagonal matrix as $t \rightarrow \infty$. The same is true if $T$ is a full symmetric matrix

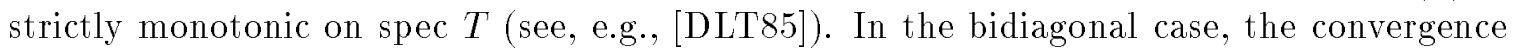
of $T(t)=A(t)^{T} A(t)$ in turn implies that $A(t)$ also converges to a diagonal matrix (see [Chu86]). For the reader's convenience we will present a (new) proof of the convergence of $A(t)$ Ccalculating en route the leading asymptotics as $t \rightarrow \infty$. By Theorem $7.14 \Gamma$ this of course gives an independent proof of the convergence of SVD and QR.

To fix notation Tlet

$$
A=B=\left(\begin{array}{cccc}
a_{1} & b_{1} & & \bigcirc \\
& \ddots & \ddots & \\
& & & b_{n-1} \\
\bigcirc & & & a_{n}
\end{array}\right), \quad a_{i}, b_{i}>0
$$

with singular values $\sigma_{1}>\sigma_{2}>\ldots>\sigma_{n}>0$ Tand let

$$
T=B^{T} B=\left(\begin{array}{cccc}
c_{1} & d_{1} & & \bigcirc \\
d_{1} & & \ddots & \\
& \ddots & \ddots & d_{n-1} \\
\bigcirc & & d_{n-1} & c_{n}
\end{array}\right),
$$


so that

$$
\begin{gathered}
c_{i}=a_{i}^{2}+b_{i-1}^{2}, \quad 1 \leq i \leq n, \\
d_{i}=a_{i} b_{i}, \quad 1 \leq i \leq n \Leftrightarrow 1 .
\end{gathered}
$$

(Here $b_{0} \equiv 0$.) Let $v_{i}=\left(v_{i}(1), \ldots, v_{i}(n)\right)^{T} \Gamma 1 \leq i \leq n$ Tdenote the unit right singular vectors for $B \Gamma T v_{i}=\sigma_{i}^{2} v_{i}$ Tnormalized so that $v_{i}(1)>0$. Set $b^{2}=\sum_{i=1}^{n-1} b_{i}^{2}$.

Theorem 7.18 (Asymptotics for SVD). Let $B(t)$ be the solution of (7.1) with $B(0)=B_{0}$ bidiagonal and $F(x)$ increasing on spec $B^{T} B$. Then as $t \rightarrow \infty$

$$
a_{j}(t)=\sigma_{j}\left(1+\frac{b_{j}^{2}}{2\left(\sigma_{j+1}^{2} \Leftrightarrow \sigma_{j}^{2}\right)}\left(1+O\left(b^{2}\right)\right)+\frac{b_{j-1}^{2}}{2\left(\sigma_{j-1}^{2} \Leftrightarrow \sigma_{j}^{2}\right)}\left(1+O\left(b^{2}\right)\right)\right)
$$

and

$$
b_{j}(t) \sim \frac{1}{\sigma_{j}}\left[\frac{\prod_{k \leq j}\left(\sigma_{k}^{2} \Leftrightarrow \sigma_{j+1}^{2}\right)}{\prod_{k \leq j-1}\left(\sigma_{k}^{2} \Leftrightarrow \sigma_{j}^{2}\right)}\right]\left[\frac{v_{j+1}(1)}{v_{j}(1)}\right] e^{\left(F\left(\sigma_{j+1}^{2}\right)-F\left(\sigma_{j}^{2}\right)\right) t}
$$

$\left(\right.$ Here $\prod_{k \leq j-1}\left(\sigma_{k}^{2} \Leftrightarrow \sigma_{j}^{2}\right) \equiv 1$ for $\left.j=1\right)$.

Proof. From (7.7) and (7.3) Г

$$
\begin{aligned}
v_{j}(1, t) & =\left(e_{1}, Q_{1}^{T}(t) v_{j}\right) \\
& =\left(\left(R_{1}(t)\right)^{-1} e_{1}, e^{t F\left(T_{0}\right)} v_{j}\right) \\
& =\frac{e^{t F\left(\sigma_{j}^{2}\right)}}{\left(R_{1}(t)\right)_{11}} v_{j}(1)
\end{aligned}
$$

and hence

$$
v_{j}(1, t)=\frac{e^{t F\left(\sigma_{j}^{2}\right)} v_{j}(1)}{\left(\sum_{m=1}^{n} e^{2 t F\left(\sigma_{m}^{2}\right)} v_{m}^{2}(1)\right)^{1 / 2}} .
$$

In particular $\Gamma$ as $F(x)$ is increasing $\Gamma$

$$
v_{j}(1, t) \rightarrow \delta_{j 1}
$$

as $t \rightarrow \infty$.

Now recall (see e.g. $[\mathrm{GvL}]$ ) that the rows

$$
\begin{array}{llll}
v_{1}(1) & v_{2}(1) & \cdots & v_{n}(1) \\
v_{1}(2) & v_{2}(2) & \cdots & v_{n}(2) \\
& \vdots & &
\end{array}
$$

of the matrix of eigenvectors $V$ for the tridiagonal matrix $T$ can be computed by applying the Gram-Schmidt procedure to the row vectors

$$
\begin{array}{llll}
v_{1}(1) & v_{2}(1) & \ldots & v_{n}(1) \\
\sigma_{1}^{2} v_{1}(1) & \sigma_{2}^{2} v_{2}(1) & \ldots & \sigma_{n}^{2} v_{n}(1) \\
& \vdots & & \\
\sigma_{1}^{2(k-1)} v_{1}(1) & \sigma_{2}^{2(k-1)} v_{2}(1) & \ldots & \sigma_{n}^{2(k-1)} v_{n}(1) .
\end{array}
$$


In particular if $v(1, t)=\left(v_{1}(1, t), \ldots, v_{n}(1, t)\right) \Gamma v(2, t)=\left(v_{1}(2, t), \ldots, v_{n}(2, t)\right)$ denote the first two rows of the matrix of eigenvectors $V(t)$ for $T(t)$; then

$$
1=(v(1, t) \wedge v(2, t), v(1, t) \wedge v(2, t))=\sum_{i<k}\left(\left|\begin{array}{cc}
v_{i}(1, t) & v_{k}(1, t) \\
v_{i}(2, t) & v_{k}(2, t)
\end{array}\right|\right)^{2} .
$$

But by Gram-Schmidt $v_{m}(2, t)=\lambda_{1}(t) \sigma_{m}^{2} v_{m}(1, t)+\lambda_{2}(t) v_{m}(1, t)$ where $\lambda_{1}(t)$ and $\lambda_{2}(t)$ are independent of $m \Gamma \lambda_{1}(t) \neq 0$. Using (7.21) we find

$$
\frac{\left|\begin{array}{ll}
v_{i}(1, t) & v_{k}(1, t) \\
v_{i}(2, t) & v_{k}(2, t)
\end{array}\right|^{2}}{\left|\begin{array}{ll}
v_{1}(1, t) & v_{2}(1, t) \\
v_{1}(2, t) & v_{2}(2, t)
\end{array}\right|^{2}}=\frac{\left|\begin{array}{ll}
1 & 1 \\
\sigma_{i}^{2} & \sigma_{k}^{2}
\end{array}\right|^{2}}{\left|\begin{array}{ll}
1 & 1 \\
\sigma_{1}^{2} & \sigma_{2}^{2}
\end{array}\right|^{2}}\left(\frac{v_{i}(1) v_{k}(1)}{v_{1}(1) v_{2}(1)}\right)^{2} e^{2\left[F\left(\sigma_{i}^{2}\right)+F\left(\sigma_{k}^{2}\right)-F\left(\sigma_{1}^{2}\right)-F\left(\sigma_{2}^{2}\right)\right] t},
$$

which $\Gamma$ by the monotonicity of $F$ Tconverges to zero as $t \rightarrow \infty$ Tunless $i=1$ and $k=2$. Thus

$$
\left|\begin{array}{ll}
v_{1}(1, t) & v_{2}(1, t) \\
v_{1}(2, t) & v_{2}(2, t)
\end{array}\right| \Leftrightarrow \pm 1
$$

and as $\left|v_{1}(2, t)\right| \leq 1$ and $v_{2}(1, t) \rightarrow 0$ by $(7.22)$ Twe conclude that

$$
v_{j}(2, t) \rightarrow \pm \delta_{j 2}
$$

Continuing by induction we learn that

$$
v_{j}(k, t) \rightarrow \pm \delta_{j k}
$$

as $t \rightarrow \infty$ Tand hence

$$
T(t)=V(t) \Sigma^{2} V(t) \rightarrow \Sigma^{2}=\operatorname{diag}\left(\sigma_{1}^{2}, \ldots, \sigma_{n}^{2}\right) .
$$

In particular $d_{i}(t)=a_{i}(t) b_{i}(t) \rightarrow 0$. But $\operatorname{det} T(t)=(\operatorname{det} A(t))^{2}=\prod_{i=1}^{n} a_{i}^{2}(t)=$ constant $\neq$ 0 Tas (7.11) is isospectral Tand $\max _{i} a_{i}(t)$ is bounded by (7.5) Twhich implies $\|A(t)\|=\left\|A_{0}\right\|$. It follows that $b_{i}(t) \rightarrow 0$ Tand from $(7.17)$ (and $\left.(7.8)\right) \Gamma$

$$
a_{i}(t)=\sqrt{c_{i}(t) \Leftrightarrow b_{i-1}^{2}(t)} \rightarrow \sigma_{i} .
$$

The eigenvalue equation for $T(t)$ implies

$$
v_{i}(k+1, t)=\frac{v_{i}(1, t)}{\left(\prod_{m=1}^{k} d_{m}\right)} \operatorname{det}\left(\sigma_{i}^{2} \Leftrightarrow T(t)\right)_{k}
$$

where $\left(\sigma_{i}^{2} \Leftrightarrow T(t)\right)_{k}$ is $k \times k$ matrix formed from the first $k$ rows and columns of $\sigma_{i}^{2} \Leftrightarrow T(t)$.

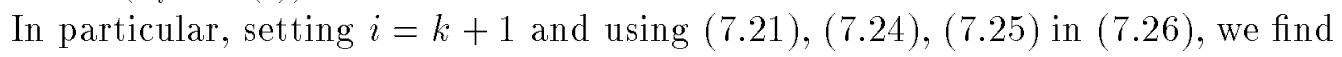

$$
\prod_{m=1}^{k} d_{m}(t) \sim \pm \frac{v_{k+1}(1)}{v_{1}(1)}\left(\prod_{m=1}^{k}\left(\sigma_{k+1}^{2} \Leftrightarrow \sigma_{m}^{2}\right)\right) e^{\left(F\left(\sigma_{k+1}^{2}\right)-F\left(\sigma_{1}^{2}\right)\right) t},
$$

which leads directly to $(7.20)$. 
To obtain the asymptotics for $a_{i}(t) \Gamma$ conjugate the eigenvalue equation for $T$ using a diagonal matrix to the form

$$
\left(\begin{array}{lllll}
c_{1} \Leftrightarrow \sigma_{i}^{2} & d_{1}^{2} & & & \\
1 & \left(c_{2} \Leftrightarrow \sigma_{i}^{2}\right) & d_{2}^{2} & & \\
& \ddots & \ddots & \ddots & \\
& & 1 & \left(c_{n-1} \Leftrightarrow \sigma_{i}^{2}\right) & d_{n-1}^{2} \\
& & & 1 & \left(c_{n} \Leftrightarrow \sigma_{i}^{2}\right)
\end{array}\right)\left(\begin{array}{l}
\tilde{v}_{i}(1) \\
\vdots \\
\\
\tilde{v}_{i}(1)
\end{array}\right)=0
$$

Applying Cramer's rule to the leading $(i \Leftrightarrow 1) \times(i \Leftrightarrow 1)$ matrix and to the trailing $(n \Leftrightarrow i) \times(n \Leftrightarrow i)$ matrixTone sees easily that

$$
\tilde{v}_{i}(i \Leftrightarrow 1) / \tilde{v}_{i}(i)=\frac{d_{i-1}^{2}}{\sigma_{i}^{2} \Leftrightarrow \sigma_{i-1}^{2}}\left(1+O\left(\sum_{j} d_{j}^{2}\right)\right)
$$

and

$$
\tilde{v}_{i}(i+1) / \tilde{v}_{i}(i)=\frac{1}{\sigma_{i}^{2} \Leftrightarrow \sigma_{i+1}^{2}}\left(1+O\left(\sum_{j} d_{j}^{2}\right)\right)
$$

respectively. Inserting these relations in the $i^{\text {th }}$ equation

$$
c_{i} \Leftrightarrow \sigma_{i}^{2}=\Leftrightarrow \frac{\tilde{v}_{i}(i \Leftrightarrow 1)}{\tilde{v}_{i}(i)} \Leftrightarrow d_{i}^{2} \frac{\tilde{v}_{i}(i+1)}{\tilde{v}_{i}(i)}
$$

and using (7.16) Tthe result follows.

Remark 7.27 From (7.24) $\Gamma v_{j}(k, t) \rightarrow \pm \delta_{j k}$ as $t \rightarrow \infty$. The choice of signs can be determined from (7.26). Indeed as $t \rightarrow \infty \Gamma v_{k+1}(k+1, t) \sim($ pos. $) \times \prod_{i=1}^{k}\left(\sigma_{k+1}^{2} \Leftrightarrow \sigma_{i}^{2}\right) \Gamma$ and so

$$
v_{j}(k, t) \rightarrow(\Leftrightarrow 1)^{k+1} \delta_{j k} .
$$

Remark 7.28 The form of (7.19) suggests a proof using more standard techniques in numerical analysis. Indeed (7.19) can easily be proved by applying two consecutive Jacobi rotations in the planes $(j \Leftrightarrow 1, j)$ and $(j, j+1)$ respectively $\Gamma$ and then using second order perturbation theory.

Remark 7.29 Note that a linearization of (7.1) around the equilibrium point $A(\infty)=$ $\operatorname{diag}\left(\sigma_{1}, \ldots, \sigma_{n}\right) \Gamma$ would also give the asymptotic rates $(7.19)$ and $(7.20)$ Tbut without the precise constants.

Remark 7.30 The asymptotics in (7.20) can be used to calculate the scattering matrix for the classical Toda lattice (see [Mos75]) directly. 


\section{The Hamiltonian Structure for the Flows}

In this section we show that equation (7.1) is Hamiltonian. More precisely there is a Poisson bracket $\{\cdot, \cdot\}_{S} \Gamma$ the Sklyanin bracket $\Gamma$ defined on the space of matrices $\Gamma$ and a Hamiltonian $H_{F}$ एsuch that the differential equations generated by $\left(H_{F},\{\cdot, \cdot\}_{S}\right) \Gamma$

$$
\frac{d}{d t} \phi(A(t))=\left\{\phi, H_{F}\right\}_{S}(A(t)), \quad A(0)=A_{0},
$$

for all observables $\phi$ Tare equivalent to (7.1).

A general reference for the Hamiltonian mechanics used in this paper is [Arn78]; a description of the Sklyanin bracket can be found in [Sem84]. The Sklyanin bracket can be defined in great generality on groups and on associative algebras (see also [LPar]) $\Gamma$ but we will restrict ourselves to the case where the underlying space is $M_{n}(\mathbf{R}) \Gamma$ the algebra of real $n \times n$ matrices.

We begin with some notation and definitions. The space $M_{n}(\mathbf{R})$ carries a natural $(g l(n, \mathbf{R})$-ad- $)$ invariant pairing

$$
\begin{gathered}
(A, B) \equiv \operatorname{tr} A B, \\
(A,[B, C])=\Leftrightarrow([B, A], C),
\end{gathered}
$$

where $A, B, C$ belong to $M_{n}(\mathbf{R})$ and $[\cdot, \cdot]$ denotes the standard commutator. Denote by $X_{a}\left(\operatorname{resp} . \widetilde{X}_{a}\right)$ the $(G l(n, \mathbf{R})-)$ left-invariant $((G l(n, \mathbf{R})-)$ right-invariant $\Gamma$ resp. $)$ vector field generated by $a \in M_{n}(\mathbf{R})$. Thus for smooth functions $\phi: M_{n}(\mathbf{R}) \rightarrow \mathbf{R} \Gamma$

$$
\begin{aligned}
& X_{a} \phi(g)=\left.\frac{d}{d t}\right|_{t=0} \phi\left(g e^{t a}\right) \equiv\left(D^{\prime} \phi(g), a\right) \\
& \tilde{X}_{a} \phi(g)=\left.\frac{d}{d t}\right|_{t=0} \phi\left(e^{t a} g\right) \equiv(D \phi(g), a)
\end{aligned}
$$

for all $g \in M_{n}(\mathbf{R})$. Clearly

$$
\begin{aligned}
& D^{\prime} \phi(g)=(\nabla \phi(g))^{T} g \\
& D \phi(g)=g(\nabla \phi(g))^{T},
\end{aligned}
$$

where $\nabla \phi(g)$ is the matrix with entries $\frac{\partial \phi}{\partial g_{i j}}(g)$. Standard computations for Lie brackets show that

$$
\begin{gathered}
{\left[X_{a}, X_{b}\right]=X_{[a, b]}} \\
{\left[\tilde{X}_{a}, \tilde{X}_{b}\right]=\Leftrightarrow \tilde{X}_{[a, b]},}
\end{gathered}
$$

and

$$
\left[X_{a}, \tilde{X}_{b}\right]=0 .
$$

Finally a linear map $R: M_{n}(\mathbf{R}) \rightarrow M_{n}(\mathbf{R})$ is said to solve the modified Yang-Baxter equation $(m Y B)$ if

$$
[R(A), R(B)] \Leftrightarrow R([A, R(B)]+[R(A), B])=\Leftrightarrow[A, B]
$$

for all $A, B \in M_{n}(\mathbf{R})$. (Such an $\mathrm{R}$ is an example of a classical r-matrix - see [Sem84]). 
Theorem 8.12 Suppose $R$ and $R^{\prime}$ are skew symmetric

$$
(A, R(B))=\Leftrightarrow(R(A), B), \quad\left(A, R^{\prime}(B)\right)=\Leftrightarrow\left(R^{\prime}(A), B\right)
$$

solutions of $(m Y B)$. Then

$$
\{\phi, \psi\}_{R, R^{\prime}}(g) \equiv(R(D \phi(g)), D \psi(g))+\left(R^{\prime}\left(D^{\prime} \phi(g)\right), D^{\prime} \psi(g)\right)
$$

defines a Poisson bracket on $M_{n}(\mathbf{R})$.

Proof. The only point to check is the Jacobi identity $\left\{\phi_{1},\left\{\phi_{2}, \phi_{3}\right\}_{R, R^{\prime}}\right\}_{R, R^{\prime}}+$ cyclic permutations (c.p.) $=0$. Set

$$
\begin{aligned}
& \{\phi, \psi\}_{r}(g) \equiv(R(D \phi(g)), D \psi(g)) \\
& \{\phi, \psi\}_{\ell}(g) \equiv\left(R^{\prime}\left(D^{\prime} \phi(g), D^{\prime} \psi(g)\right)\right.
\end{aligned}
$$

We have

$$
\begin{aligned}
\left(D^{\prime}\left\{\phi_{2}, \phi_{3}\right\}_{\ell}(g), a\right) & =\left.\frac{d}{d t}\right|_{t=0}\left\{\phi_{2}, \phi_{3}\right\}_{\ell}\left(g e^{t a}\right) \\
& =\left(R^{\prime}\left(D^{\prime} \phi_{2}(g)\right),\left.\frac{d}{d t}\right|_{t=0} D^{\prime} \phi_{3}\left(g e^{t a}\right)\right) \Leftrightarrow\left(\left.\frac{d}{d t}\right|_{t=0} D^{\prime} \phi_{2}\left(g e^{t a}\right), R^{\prime}\left(D^{\prime} \phi_{3}(g)\right)\right) \\
& =X_{a} X_{R^{\prime}\left(D^{\prime} \phi_{2}(g)\right)} \phi_{3}(g) \Leftrightarrow X_{a} X_{R^{\prime}\left(D^{\prime} \phi_{3}(g)\right)} \phi_{2}(g),
\end{aligned}
$$

which implies

$$
\begin{aligned}
\left\{\phi_{1},\left\{\phi_{2}, \phi_{3}\right\}_{\ell}\right\}_{\ell}+\text { c.p. } & =\frac{1}{2}\left(R^{\prime}\left(D^{\prime} \phi_{1}\right), D^{\prime}\left\{\phi_{2}, \phi_{3}\right\}_{2}\right)+\text { c.p. } \\
& =X_{R^{\prime}\left(D^{\prime} \phi_{1}\right)} X_{R^{\prime}\left(D^{\prime} \phi_{2}\right)} \phi_{3} \Leftrightarrow X_{R^{\prime}\left(D^{\prime} \phi_{1}\right)} X_{R^{\prime}\left(D^{\prime} \phi_{3}\right)} \phi_{2}+\text { c.p. } \\
& =\left[X_{R^{\prime}\left(D^{\prime} \phi_{2}\right)}, X_{R^{\prime}\left(D^{\prime} \phi_{3}\right)}\right] \phi_{1}+\text { c.p. } \\
& =X_{\left[R^{\prime}\left(D^{\prime} \phi_{2}\right), R^{\prime}\left(D^{\prime} \phi_{3}\right)\right]} \phi_{1}+\text { c.p. }, \quad \text { by }(8.8) \Gamma \\
& =\left(D^{\prime} \phi_{1},\left[R^{\prime}\left(D^{\prime} \phi_{2}\right), R^{\prime}\left(D^{\prime} \phi_{3}\right)\right]\right)+\text { c.p. }
\end{aligned}
$$

Similarly

$$
\left\{\phi_{1},\left\{\phi_{2}, \phi_{3}\right\}_{r}\right\}_{r}=\Leftrightarrow\left(D \phi_{1},\left[R\left(D \phi_{2}\right), R\left(D \phi_{3}\right)\right]\right)+\text { c.p. } \Gamma
$$

and

$$
\begin{aligned}
\left\{\phi_{1},\left\{\phi_{2}, \phi_{3}\right\}_{r}\right\}_{\ell}+\left\{\phi_{1},\left\{\phi_{2}, \phi_{3}\right\}_{\ell}\right\}_{r} & =\left[X_{R^{\prime}\left(D^{\prime} \phi_{2}\right)}, \tilde{X}_{R\left(D \phi_{3}\right)}\right] \phi_{1}+\text { c.p. } \\
& =0, \quad \text { by }(8.10) \Gamma
\end{aligned}
$$

Thus

$$
\begin{aligned}
\left\{\phi_{1},\left\{\phi_{2}, \phi_{3}\right\}_{R, R^{\prime}}\right\}_{R, R^{\prime}}+\text { c.p. } & =\left\{\phi_{1},\left\{\phi_{2}, \phi_{3}\right\}_{\ell}\right\}_{\ell}+\left\{\phi_{1},\left\{\phi_{2}, \phi_{3}\right\}_{r}\right\}_{r}+\text { c.p. } \\
& =\left(D^{\prime} \phi_{1},\left[R^{\prime}\left(D^{\prime} \phi_{2}\right), R^{\prime}\left(D^{\prime} \phi_{3}\right)\right]\right) \Leftrightarrow\left(D \phi_{1},\left[R\left(D \phi_{2}\right), R\left(D \phi_{3}\right)\right]\right)+\text { c.p. } \\
& =\Leftrightarrow\left(D^{\prime} \phi_{1},\left[D^{\prime} \phi_{2}, D^{\prime} \phi_{3}\right]\right)+\left(D \phi_{1},\left[D \phi_{2}, D \phi_{3}\right]\right)
\end{aligned}
$$

where in the last step we have used $(m Y B) \Gamma(8.13)$ and (8.3). Direct substitution of (8.6) and (8.7) show that the last two terms cancel $\Gamma$ and this proves the theorem. 
Set

$$
R(A) \equiv A_{+} \Leftrightarrow A_{-}, \quad R^{\prime}(A) \equiv \Leftrightarrow R(A)
$$

where $A_{+}$is the strict upper part of $A$ and $A_{-}$is strict lower part of $A$ as before. A straightforward calculation shows that $R$ and $R^{\prime}$ are skew and solve $(m Y B)$.

For SVDTthe Sklyanin bracket is defined by

$$
\begin{aligned}
\{\phi, \psi\}_{S}(A) & \equiv\{\phi, \psi\}_{R,-R}(A) \\
& =(R(D \phi(A)), D \psi(A)) \Leftrightarrow\left(R\left(D^{\prime} \phi(A)\right),\left(D^{\prime} \psi(A)\right)\right)
\end{aligned}
$$

Remark 8.17 The $R$ matrix in (8.15) also arises in the study of the Cholesky eigenvalue algorithm (see [DLT89]).

By Theorem $8.12 \Gamma\{\cdot, \cdot\}_{S}$ gives a Poisson bracket on $M_{n}(\mathbf{R})$. But more is true: $\{\cdot, \cdot\}_{S}$ restricts as a Poisson bracket to the submanifolds

$$
\left\{A \in M_{n}(\mathbf{R}): \operatorname{det} A=c\right\}
$$

for any constant $c \neq 0$. Indeed $\Gamma$ from the formula $(\nabla \log \operatorname{det} A)^{T}=A^{-1} \Gamma$ we see that $D \log \operatorname{det}(A)=D^{\prime} \log \operatorname{det}(A)=I$ T which implies $R(D \log \operatorname{det}(A))=R\left(D^{\prime} \log \operatorname{det} A\right)=0$. Hence $\{\text { det, } \phi\}_{S}=0$ for all functions $\phi$.

We now show that (7.1) is Hamiltonian.

Theorem 8.18 Let $F(x)$ be a smooth real-valued function on $(0, \infty)$ and let $G_{F}(x)=$ $\Leftrightarrow \int^{x} \frac{F(s)}{2 s} d s$ be a primitive of $\Leftrightarrow F(x) / 2 x$. Then the equation

$$
\frac{d}{d t} \phi(A(t))=\left\{\phi, H_{F}\right\}_{S}(A(t)), \quad A(0)=A_{0},
$$

generated by $H_{F}(A)=\operatorname{tr} G_{F}\left(A^{T} A\right)$ on $\left\{A \in M_{n}(\mathbf{R}): \operatorname{det} A=\operatorname{det} A_{0} \neq 0\right\}$, is equivalent to $(7.1)$.

Proof. From (8.17) Г

$$
\dot{\phi}=\left\{\phi, H_{F}\right\}_{S}=\Leftrightarrow\left(\nabla \phi^{T}(A),\left(R\left(A \nabla H_{F}^{T}(A)\right)\right) A\right)+\left(\nabla \phi^{T}(A), A R\left(\nabla H_{F}^{T}(A) A\right)\right),
$$

so that

$$
\dot{A}=A R\left(\nabla H_{F}^{T}(A) A\right) \Leftrightarrow\left(R\left(A \nabla H_{F}^{T}(A)\right)\right) A .
$$

But by differentiation $\Gamma$

$$
\begin{aligned}
\nabla H_{F}(A) & =2 A G_{F}^{\prime}\left(A^{T} A\right) \\
& =\Leftrightarrow A\left(A^{T} A\right)^{-1} F\left(A^{T} A\right) \\
& =\Leftrightarrow\left(A^{T}\right)^{-1} F\left(A^{T} A\right),
\end{aligned}
$$

which implies

$$
\dot{A}=\Leftrightarrow A R\left(F\left(A^{T} A\right)\right)+R\left(F\left(A A^{T}\right)\right) A .
$$

As $\pi_{0}(S)=\Leftrightarrow R(S)$ for any symmetric matrix $S$ Tthis proves the theorem. 
In particular

$$
H_{S V D}(A) \equiv \Leftrightarrow \frac{1}{4} \operatorname{tr}\left(\log \left(A^{T} A\right)\right)^{2}
$$

generates the SVD flow. Also $H=\Leftrightarrow \frac{1}{2} \operatorname{tr}\left(A^{T} A\right)$ generates the Toda-SVD flow in [Chu86].

The bracket $\{\cdot, \cdot\}_{S}$ is highly degenerate and the determination of the associated symplectic leaves (see [Wei85] $[$ Sem85]) is in general extremely difficult. We haveThoweverTthe following happy fact.

Theorem 8.21 The set $\mathcal{B}_{\Delta}$ of bidiagonal matrices $B$ with positive entries $a_{p}, b_{q}$ and fixed determinant,

$$
\operatorname{det} B=\prod_{p=1}^{n} a_{p}=\Delta
$$

is a $(2 n \Leftrightarrow 2)$-dimensional symplectic leaf for the Sklyanin bracket $\{\cdot, \cdot\}_{S}$. Moreover, $\mathcal{B}_{\Delta}$ has a global Darboux coordinate system given by

$$
\begin{gathered}
x_{i} \equiv \log b_{i}, \quad 1 \leq i \leq n \Leftrightarrow 1, \\
y_{i} \equiv \log \prod_{j=1}^{i} a_{j}, \quad 1 \leq i \leq n \Leftrightarrow 1, \\
\left\{x_{i}, x_{j}\right\}_{S}=0, \quad\left\{y_{i}, y_{j}\right\}_{S}=0, \quad\left\{x_{i}, y_{j}\right\}_{S}=\delta_{i j} .
\end{gathered}
$$

Proof. We compute $\{\phi, \psi\}_{S}(B)$ at a bidiagonal matrix $B$. Set $\eta(m)=\Leftrightarrow 1,0,1$ if $m$ is negative $\mathrm{Ezero}$ or positive respectively.

Insert the formulae

$$
\begin{aligned}
\left(B \nabla \phi^{T}(B)\right)_{i j} & =a_{i} \phi_{j i}+b_{i} \phi_{j, i+1} \\
\left(R\left(B \nabla \phi^{T}(B)\right)\right)_{i j} & =\eta(j \Leftrightarrow i)\left(a_{i} \phi_{j i}+b_{i} \phi_{j, i+1}\right) \\
\left(\nabla \phi^{T}(B) B\right)_{i j} & =a_{j} \phi_{j i}+b_{j-1} \phi_{j-1, i} \\
\left(R\left(\nabla \phi^{T}(B) B\right)\right)_{i j} & =\eta(j \Leftrightarrow i)\left(a_{j} \phi_{j i}+b_{j-1} \phi_{j-1, i}\right)
\end{aligned}
$$

and their analogs for $\psi$ Tinto (8.17) Tto obtain after some algebra

$$
\begin{aligned}
\{\phi, \psi\}_{S}(B)= & \sum_{i, j}(\eta(j \Leftrightarrow i) \Leftrightarrow \eta(j+1 \Leftrightarrow i)) a_{i} b_{j} \phi_{j i} \psi_{i, j+1} \\
& \quad+\sum_{i, j}(\eta(j \Leftrightarrow i) \Leftrightarrow \eta(j+1 \Leftrightarrow i)) a_{j} b_{i} \phi_{j, i+1} \psi_{i j} \\
= & \sum_{i}\left(\Leftrightarrow a_{i} b_{i-1} \phi_{i-1, i} \psi_{i i} \Leftrightarrow a_{i} b_{i} \phi_{i i} \psi_{i, i+1}+a_{i} b_{i} \psi_{i, i+1} \psi_{i i}+a_{i+1} b_{i} \phi_{i+1, i+1} \psi_{i, i+1}\right) .
\end{aligned}
$$

Changing variables $\Gamma a, b \rightarrow x, y$ Ton $\mathcal{B}_{\Delta}$ now leads to

$$
\{\phi, \psi\}_{S}(B)=\{\phi, \psi\}_{S}(B(x, y))=\sum_{i=1}^{n-1}\left(\frac{\partial \phi}{\partial x_{i}} \frac{\partial \psi}{\partial y_{i}} \Leftrightarrow \frac{\partial \phi}{\partial y_{i}} \frac{\partial \psi}{\partial x_{i}}\right),
$$

which is the canonical non-degenerate Poisson bracket on $\mathbf{R}^{2 n-2}$ Tand (8.25) follows. 
For later convenience $\Gamma$ we introduce the notation

$$
y_{n} \equiv \log \prod_{j=1}^{n} a_{j}=\log \Delta
$$

Remark 8.28 Formula (8.26) makes explicit the fact that $\mathcal{B}_{\Delta}$ is a symplectic leaf of $\{\cdot, \cdot\}_{S}$. In particular $\{\phi, \psi\}_{S}(B(x, y))$ depends only on the values of $\phi$ and $\psi$ on $\mathcal{B}_{\Delta}$.

Remark 8.29 The fact that $\mathcal{B}_{\Delta}$ is a natural phase space for the Hamiltonian version of SVD is in striking parallel to the fact that $T_{\tau} \Gamma$ the tridiagonal matrices with prescribed trace $\tau$ (and with nonzero off diagonal elements) $\Gamma$ provides a natural phase space for the Hamiltonian version of $\mathrm{QR}$. The relevant Poisson structure for $\mathrm{QR}$ is given by the LiePoisson structure on the dual of the Lie algebra of the lower triangular group (see [Kos79] $\mathrm{\Gamma}$ [Ad179]; see also [DLNT86]). HoweverTthe map $B \mapsto B^{T} B$ from $\mathcal{B}_{\Delta}$ to $T_{\tau}$ is not symplectic and the relationship between the two Poisson structures is not clear. On the other hand the perfect shuffle $B \mapsto S$ of Section 2Tinduces a Poisson structure on the space of tridiagonal matrices $S$ Tof type (2.2) Twith fixed determinant. In particular Tthis shows that the Kac-van Moerbeke lattice (see Remark 7.12) is Hamiltonian on the space of such matrices $S$.

We conclude this section by proving Fact 1 of the Introduction. Thus Tif $M(j, i)$ is the Jacobian of the iterated SVD map from $B_{i}$ to $B_{j}$ expressed in the variables $\log b_{1}, \ldots, \log b_{n-1} \Gamma$ $\log a_{1}, \ldots, \log a_{n}$ Tthen

$$
\lambda \in \operatorname{spec} M(j, i) \Leftrightarrow \lambda^{-1} \in \operatorname{spec} M(j, i)
$$

For a bidiagonal matrix $B$ set

$$
\begin{gathered}
\beta_{i}=\log b_{i}, \quad 1 \leq i \leq n \Leftrightarrow 1, \\
\alpha_{i}=\log a_{i}, \quad 1 \leq i \leq n .
\end{gathered}
$$

so that

$$
\left(\begin{array}{l}
\beta \\
\alpha
\end{array}\right)=\left(\begin{array}{ll}
1 & 0 \\
0 & N
\end{array}\right)\left(\begin{array}{l}
x \\
y
\end{array}\right)
$$

where $N$ is the $n \times n$ matrix $\left(\begin{array}{cccc}1 & & & 0 \\ \Leftrightarrow 1 & \ddots & & \\ & \ddots & \ddots & \\ 0 & & \Leftrightarrow 1 & 1\end{array}\right)$. Note $N^{-1}=\left(\begin{array}{cccc}1 & & & 0 \\ 1 & 1 & & \\ \vdots & \ddots & & \\ & & 1 & \\ 1 & 1 & 1 & 1\end{array}\right)$. Let $\left(\begin{array}{c}\beta^{i} \\ \alpha^{i}\end{array}\right)=\left(\begin{array}{ll}1 & 0 \\ 0 & N\end{array}\right)\left(\begin{array}{l}x^{i} \\ y^{i}\end{array}\right) \Gamma\left(\begin{array}{l}\beta^{j} \\ \alpha^{j}\end{array}\right)=\left(\begin{array}{ll}1 & 0 \\ 0 & N\end{array}\right)\left(\begin{array}{l}x^{j} \\ y^{j}\end{array}\right)$ be the coordinates of the $i^{\text {th }}$ and $j^{\text {th }}$ SVD iterates $B_{i}$ and $B_{j}$ Twith $i<j$. Then

$$
M(j, i) \equiv \frac{\partial\left(\beta^{j}, \alpha^{j}\right)}{\partial\left(\beta^{i}, \alpha^{i}\right)}=\left(\begin{array}{ll}
1 & 0 \\
0 & N
\end{array}\right) \frac{\partial\left(x^{j}, y^{j}\right)}{\partial\left(x^{i}, y^{i}\right)}\left(\begin{array}{ll}
1 & 0 \\
0 & N^{-1}
\end{array}\right) .
$$


Under the iteration $\Gamma y_{n}^{j}=\log \operatorname{det} B^{j}=\log \operatorname{det} B^{i}=y_{n}^{i}$. Hence

$$
\frac{\partial\left(x^{j}, y^{j}\right)}{\partial\left(x^{i}, y^{i}\right)}=\left(\begin{array}{cc} 
& \frac{\partial x_{1}^{j}}{\partial y_{n}^{2}} \\
\frac{\partial\left(x_{1}^{j}, \ldots, x_{n-1}^{j}, y_{1}^{j}, \ldots, y_{n-1}^{j}\right)}{\partial\left(x_{1}^{2}, \ldots, x_{n-1}^{2}, y_{1}^{2}, \ldots, y_{n-1}^{2}\right)} & \vdots \\
0 \cdots 0 & \frac{\partial y_{n-1}^{j}}{\partial y_{n}^{2}} \\
0 & 1
\end{array}\right) .
$$

Now recall the following standard fact from Hamiltonian mechanics ([Ar]): let $J$ denote the standard matrix $\left(\begin{array}{rr}0 & I \\ \Leftrightarrow I & 0\end{array}\right)$ and suppose $(x(t ; x, y), y(t ; x, y))$ is the solution of a Hamiltonian system of equations in $\mathbf{R}^{2 n-2}$ in canonical form

$$
\frac{d}{d t}\left(\begin{array}{l}
x \\
y
\end{array}\right)=J \nabla H=\left(\begin{array}{ll}
0 & I \\
\Leftrightarrow I & 0
\end{array}\right)\left(\begin{array}{l}
H_{x} \\
H_{y}
\end{array}\right), \quad(x(0 ; x, y), y(0 ; x, y))=(x, y),
$$

for some Hamiltonian $H: \mathbf{R}^{2 n-2} \rightarrow \mathbf{R}$. Then for any $t$ the Jacobian $D=\frac{\partial(x(t ; x, y), y(t ; x, y))}{\partial(x, y)}$ is symplecticri.e. $D^{T} J D=J$. But $\operatorname{det}\left(D^{T} \Leftrightarrow \lambda\right)=\operatorname{det}\left(J D^{-1} J^{-1} \Leftrightarrow \lambda\right)=\operatorname{det}\left(D^{-1} \Leftrightarrow \lambda\right)$. Thus if $D$ is symplectic $\Gamma$

$$
\lambda \in \operatorname{spec} D \Leftrightarrow \lambda^{-1} \in \operatorname{spec} D .
$$

Finally from Theorems $7.14 \Gamma 8.18$ and $8.21 \Gamma x_{1}^{j}, \ldots, x_{n-1}^{j}, y_{1}^{j}, \ldots, y_{n-1}^{j}$ is the time $t=$ $j \Leftrightarrow i$ evaluation in canonical variables of a Hamiltonian flow with initial data $x_{1}^{i}, \ldots, x_{n-1}^{i} \Gamma$ $y_{1}^{i}, \ldots, y_{n-1}^{i}$. Hence the matrix $\frac{\partial\left(x_{1}^{j}, \ldots, x_{n-1}^{j}, y_{1}^{j}, \ldots, y_{n-1}^{j}\right)}{\partial\left(x_{1}^{2}, \ldots, x_{n-1}^{2}, y_{1}^{2}, \ldots, y_{n-1}^{2}\right)}$ is symplectic and $(8.36)$ holds. Fact 1 now follows from (8.33) and (8.34).

Remark 8.37 The Hamiltonian $H_{S V D}$ on the (2n-2)-dimensional leaf $\mathcal{B}_{\Delta}$ is completely integrable in the sense of Liouville. The commuting integrals are the singular values $\sigma_{1}, \ldots, \sigma_{n-1}$ (recall $\prod_{i=1}^{n} \sigma_{i}=\Delta$ is a Casimir) Tand the associated angles are suitable combinations of the logarithms of the first components of the unit singular vectors. We leave the details to the interested reader ( $c f$. [Mos75] Г[DLNT86] Tfor example). 


\section{$9 \quad$ Asymptotics of $\|M(j, i)\|$}

By the results of Sections 7 and 8Tthe SVD flow on bidiagonal matrices $B=\left(\begin{array}{cccc}a_{1} & b_{1} & & \\ & \ddots & \ddots & \\ & & \ddots & b_{n-1} \\ & & a_{n}\end{array}\right)$ takes the form

$$
\begin{aligned}
\frac{d x_{i}}{d t} & =\frac{\partial H_{S V D}}{\partial y_{i}}\left(x_{1}, \ldots, y_{n-1} ; y_{n}\right), \quad 1 \leq i \leq n \Leftrightarrow 1, \\
\frac{d y_{i}}{d t} & =\Leftrightarrow \frac{\partial H_{S V D}}{\partial x_{i}}\left(x_{1}, \ldots, y_{n-1} ; y_{n}\right), \quad 1 \leq i \leq n \Leftrightarrow 1, \\
\frac{d y_{n}}{d t} & =0 \\
x_{i}(0) & =x_{i}, \quad y_{i}(0)=y_{i}, \quad 1 \leq i \leq n \Leftrightarrow 1, \quad y_{n}(0)=y_{n}(t)=y_{n},
\end{aligned}
$$

in the canonical coordinates $x_{i}=\log b_{i} \Gamma y_{i}=\log \prod_{j=1}^{i} a_{j} \Gamma$ where $H_{S V D}=\Leftrightarrow \frac{1}{4} \operatorname{tr}\left(\log \left(B^{T}(x, y) B(x, y)\right)\right)^{2}$. This leads to the equation

$$
\frac{d}{d t} K_{n}=\left(\begin{array}{ccc}
J & \nabla_{n}^{2} H_{S V D} \\
0 & \ldots & 0
\end{array}\right) K_{n}, K_{n}(0)=I,
$$

for the full $(2 n \Leftrightarrow 1) \times(2 n \Leftrightarrow 1)$ Jacobian matrix $K_{n}(t)=\frac{\partial\left(x_{1}(t), \ldots, x_{n-1}(t), y_{1}(t), \ldots, y_{n}(t)\right)}{\partial\left(x_{1} \ldots, x_{n-1}, y_{1} \ldots, y_{n}\right)} \Gamma$ where $J$ is again the standard $(2 n \Leftrightarrow 2) \times(2 n \Leftrightarrow 2)$ matrix $\left(\begin{array}{rr}0 & I \\ \Leftrightarrow I & 0\end{array}\right)$ and $\nabla_{n}^{2} H_{S V D}$ is the $(2 n \Leftrightarrow$ $2) \times(2 n \Leftrightarrow 1)$ Hessian matrix

$$
\begin{aligned}
& \nabla_{n}^{2} H_{S V D}=\left(\frac{\partial^{2} H_{S V D}}{\partial z_{i} \partial z_{j}}\right)_{1 \leq i \leq 2 n-2,1 \leq j \leq 2 n-1} \\
& \left(z_{1}, \ldots, z_{2 n-1}\right) \equiv\left(x_{1}, \ldots, x_{n-1}, y_{1}, \ldots, y_{n}\right) .
\end{aligned}
$$

Our goal in this section is to evaluate $K_{n}(t)$ as $t \rightarrow \infty$. By (8.33) Tthe asymptotics for $M(j, i)$ will then follow. Inserting $F(x)=\log x$ in Theorem 7.18 एwe obtain

$$
a_{i}(t)=\sigma_{i}+O\left(b^{2}\right), \quad 1 \leq i \leq n
$$

and

$$
b_{i}(t) \sim b_{i}^{\infty}\left(\frac{\sigma_{i+1}}{\sigma_{i}}\right)^{2 t}, \quad 1 \leq i \leq n \Leftrightarrow 1
$$

as $t \rightarrow \infty$ Wwhere $b_{i}^{\infty}>0$ and $b^{2}=\sum_{j=1}^{n-1} b_{j}^{2}$ as before.

A convenient formula for $H_{S V D}$ is given by the spectral representation

$$
\operatorname{tr}\left(\log B^{T} B\right)^{2}=\operatorname{tr} \int_{C} \frac{(\log s)^{2}}{s \Leftrightarrow B^{T} B} \frac{d s}{2 \pi i},
$$


where $\mathcal{C}$ is the counterclockwise contour

$$
\begin{array}{lllll}
\times & \times & \times & \ldots & \times \\
0 & \sigma_{n}^{2} & \sigma_{n-1}^{2} & \ldots & \sigma_{1}^{2} \\
& & & & \\
& \multicolumn{4}{c}{}
\end{array}
$$

from which we obtain $\Gamma$ after one integration by parts $\Gamma$

$\frac{\partial^{2}}{\partial z_{j} \partial z_{k}} \operatorname{tr}\left(\log B^{T} B\right)^{2}=\operatorname{tr} \int_{\mathcal{C}} \frac{\log s}{s}\left[\frac{\partial^{2} B^{T} B}{\partial z_{j} \partial z_{k}} \frac{1}{s \Leftrightarrow B^{T} B}+\frac{\partial B^{T} B}{\partial z_{j}} \frac{1}{s \Leftrightarrow B^{T} B} \frac{\partial B^{T} B}{\partial z_{k}} \frac{1}{s \Leftrightarrow B^{T} B}\right] \frac{d s}{\pi i}$.

Now $\partial B / \partial x_{m}=e_{m, m+1} b_{m} \Gamma 1 \leq m \leq n \Leftrightarrow 1 \Gamma$ where $e_{i j}$ is the standard $n \times n$ matrix with 1 in the $(i, j)$ position $\Gamma$ and zero elsewhere. Thus if $z_{j}$ or $z_{k}$ lies in the set $\left\{x_{1}, \ldots, x_{n-1}\right\} \Gamma$ then $\frac{\partial^{2}}{\partial z_{j} \partial z_{k}} \operatorname{tr}\left(\log B^{T} B\right)^{2}$ is of order $b$ Tand hence is exponentially decreasing. (Here we use (9.5) and (9.6) to bound $\max _{s \in \mathcal{C}}\left\|\left(S \Leftrightarrow B^{T} B\right)^{-1}\right\|$ Tetc. $)$

The leading order contribution comes from the derivatives $\partial^{2} / \partial y_{j} \partial y_{k}$. We find

$$
\frac{\partial B^{T} B}{\partial y_{j}}=2 a_{j}^{2} e_{j j} \Leftrightarrow 2 a_{j+1}^{2} e_{j+1, j+1}+O(b)
$$

and

$$
\begin{aligned}
\frac{\partial^{2} B^{T} B}{\partial y_{j} \partial y_{k}}= & \delta_{j k}\left(4 a_{j}^{2} e_{j j}+4 a_{j+1}^{2} e_{j+1, j+1}\right) \\
& +\delta_{j-1, k}\left(\Leftrightarrow 4 a_{j}^{2}\right) e_{j j}+\delta_{j+1, k}\left(\Leftrightarrow 4 a_{j+1}^{2}\right) e_{j+1, j+1} \\
& +O(b)
\end{aligned}
$$

where $a_{n+1} \equiv 0$ Tetc. Substituting (9.9) and (9.10) in (9.8) Twe find

$$
\begin{aligned}
& \frac{\partial^{2}}{\partial y_{j} \partial y_{k}} \operatorname{tr}\left(\log B^{T} B\right)=\quad \operatorname{tr} \int_{\mathcal{C}} \frac{\log s}{s}\left[\delta_{j k}\left(4 \sigma_{j}^{2} e_{j j}+4 \sigma_{j+1}^{2} e_{j+1, j+1}\right)\left(\begin{array}{ccc}
\left(s \Leftrightarrow \sigma_{1}^{2}\right)^{-1} & \\
& \ddots & \\
0 & \left(s \Leftrightarrow \sigma_{n}^{2}\right)^{-1}
\end{array}\right)\right. \\
& +\delta_{j-1, k}\left(\Leftrightarrow 4 \sigma_{j}^{2}\right) e_{i j}\left(\begin{array}{ccc}
\left(s \Leftrightarrow \sigma_{1}^{2}\right)^{-1} & & \bigcirc \\
& \ddots & \\
\bigcirc & & \left(s \Leftrightarrow \sigma_{n}^{2}\right)^{-1}
\end{array}\right) \\
& +\delta_{j+1, k}\left(\Leftrightarrow 4 \sigma_{j+1}^{2}\right) e_{j+1, j+1}\left(\begin{array}{ccc}
\left(s \Leftrightarrow \sigma_{1}^{2}\right)^{-1} & & \bigcirc \\
& \ddots & \\
\bigcirc & & \left(s \Leftrightarrow \sigma_{n}^{2}\right)^{-1}
\end{array}\right) \\
& +\left(2 \sigma_{j}^{2} e_{j j} \Leftrightarrow 2 \sigma_{j+1}^{2} e_{j+1, j+1}\right)\left(\begin{array}{ccc}
\left(s \Leftrightarrow \sigma_{1}^{2}\right)^{-1} & & \bigcirc \\
& \ddots & \\
\bigcirc & & \left(s \Leftrightarrow \sigma_{n}^{2}\right)^{-1}
\end{array}\right)
\end{aligned}
$$




$$
\begin{aligned}
& \left.\times\left(2 \sigma_{k}^{2} e_{k k} \Leftrightarrow 2 \sigma_{k+1}^{2} e_{k+1, k+1}\right)\left(\begin{array}{ccc}
\left(s \Leftrightarrow \sigma_{1}^{2}\right)^{-1} & & \bigcirc \\
& \ddots & \\
\bigcirc & & \left(s \Leftrightarrow \sigma_{n}^{2}\right)^{-1}
\end{array}\right)\right] \frac{d s}{\pi i} \\
& +O(b) .
\end{aligned}
$$

After performing the integrals $\Gamma$ this leads to

$\frac{\partial^{2}}{\partial y_{j} \partial y_{k}} \operatorname{tr}\left(\log B^{T} B\right)^{2}=16 \delta_{j k} \Leftrightarrow 8 \delta_{j-1, k} \Leftrightarrow 8 \delta_{j+1, k}+O(b), \quad 1 \leq j \leq n \Leftrightarrow 1, \quad 1 \leq k \leq n$,

and hence

$$
\left(\begin{array}{lll}
J & \nabla_{n}^{2} H_{S V D} \\
0 & \cdots & 0
\end{array}\right)=\left(\begin{array}{ll}
O_{n-1, n-1} & L \\
O_{n, n-1} & O_{n, n}
\end{array}\right)+O(b)
$$

where $L$ is the $(n \Leftrightarrow 1) \times n$ matrix

$$
L=\left(\begin{array}{rrrrrrrr}
\Leftrightarrow 4 & 2 & & & & & & 0 \\
2 & \Leftrightarrow 4 & 2 & & & & & \\
& 2 & \Leftrightarrow 4 & & & & & \\
& & \ddots & \ddots & \ddots & & & \\
& & & & & & & \\
& & & & 2 & \Leftrightarrow 4 & 2 & \\
0 & & & & & 2 & \Leftrightarrow 4 & 2
\end{array}\right)
$$

Equation (9.2) now takes the form

$$
\frac{d K_{n}}{d t}=\left(\begin{array}{cc}
0 & L \\
0 & 0
\end{array}\right) K_{n}+C(t) K_{n}, \quad K_{n}(0)=I
$$

where by $(9.6) \Gamma$

$$
\|C(t)\| \leq c_{0} e^{-\delta t}, \quad \delta=\min _{1 \leq i \leq n-1} \log \left(\sigma_{i}^{2} / \sigma_{i+1}^{2}\right)>0,
$$

for some positive constant $c_{0}$. Rewriting (9.13) in the standard way (see e.g. [CL55]) in integral form $\Gamma$ we obtain after iteration

$$
\begin{aligned}
K_{n}(t) & =e^{\left(\begin{array}{cc}
0 & L \\
0 & 0
\end{array}\right) t}\left(\begin{array}{cc}
A_{11} & A_{12} \\
A_{21} & A_{22}
\end{array}\right)(1+o(1)) \\
& =\left(\begin{array}{cc}
A_{11}+t L A_{21} & A_{12}+t L A_{22} \\
A_{21} & A_{22}
\end{array}\right)(1+o(1))
\end{aligned}
$$

for suitable constant matrices $A_{i j}$. Here the terms $o(1)$ are exponentially decreasing. Finally as in $(8.33) \Gamma$

$$
M(t, 0)=\frac{\partial(\beta(t), \alpha(t))}{\partial(\beta, \alpha)}=\left(\begin{array}{cc}
B_{11}+t \Gamma_{n} B_{21} & B_{12}+t \Gamma_{n} B_{22} \\
B_{21} & B_{22}
\end{array}\right)(1+o(1))
$$


for suitable $B_{i j}$ Twhere $\Gamma_{n}$ is the $(n \Leftrightarrow 1) \times n$ matrix $\Gamma$

$$
\Gamma_{n}=L N^{-1}=\left(\begin{array}{rrrrrr}
\Leftrightarrow 2 & 2 & & & & 0 \\
& \Leftrightarrow 2 & 2 & & & \\
& & & \ddots & \ddots & \\
& & & & \Leftrightarrow 2 & 2
\end{array}\right) \text {, }
$$

which appears in (1.9).

To compute $B_{21}, B_{22}$ Tnote that for any $t \Gamma$

$$
\sigma_{\ell}\left(B^{\prime}\right)=\sigma_{\ell}(B), \quad 1 \leq \ell<n,
$$

where $B^{\prime}=B(t)$. Thus for $1 \leq m \leq n \Leftrightarrow 1 \Gamma 1 \leq \ell \leq n \Gamma$

$$
\sum_{i=1}^{n} \frac{\partial \sigma_{\ell}\left(B^{\prime}\right)}{\partial a_{i}^{\prime}} a_{i}^{\prime} \frac{\partial \alpha_{i}^{\prime}}{\partial \beta_{m}}+\sum_{i=1}^{n-1} \frac{\partial \sigma_{\ell}\left(B^{\prime}\right)}{\partial b_{i}^{\prime}} b_{i}^{\prime} \frac{\partial \beta_{i}^{\prime}}{\partial \beta_{m}}=\frac{\partial \sigma_{\ell}}{\partial \beta_{m}} .
$$

By regular perturbation theory applied to the perfect shuffle $S$ of $B$ (see (2.2)) Twe see that (in the notation of Section 2)

$$
\begin{aligned}
\frac{\partial \sigma_{\ell}\left(B^{\prime}\right)}{\partial a_{i}^{\prime}} & =2 h_{\ell}^{+}(2 i \Leftrightarrow 1) h_{\ell}^{+}(2 i) \\
& =u_{\ell}^{\prime}(i) v_{\ell}^{\prime}(i)
\end{aligned}
$$

where $u_{\ell}^{\prime}, v_{\ell}^{\prime}$ are the unit eigenvectors of $B^{\prime}\left(B^{\prime}\right)^{T}$ and $\left(B^{\prime}\right)^{T} B^{\prime}$ respectivelyTchosen such that $u_{\ell}^{\prime}(1), v_{\ell}^{\prime}(1)>0$. But by Remark $7.27 \Gamma v_{\ell}^{\prime}(i) \rightarrow(\Leftrightarrow 1)^{i+1} \delta_{\ell i}$ as $t \rightarrow \infty$. A similar analysis shows that the same is true for $u_{\ell}^{\prime}(i)$; hence $\frac{\partial \sigma_{\ell}\left(B^{\prime}\right)}{\partial a_{i}^{\prime}} \rightarrow \delta_{\ell i}$. On the other hand $\Gamma$ by $(9.16) \Gamma$ $\partial \beta_{i}^{\prime} / \partial \beta_{m}$ grows at worst linearly as $t \rightarrow \infty$ Tbut $b_{i}^{\prime}$ decreases exponentially and $\partial \sigma_{\ell}\left(B^{\prime}\right) / \partial b_{i}^{\prime}$ is bounded Tagain by regular perturbation theory. Inserting this information into (9.18) Гwe learn that

$$
\lim _{t \rightarrow \infty} \frac{\partial \alpha_{\ell}^{\prime}}{\partial \beta_{m}}=\frac{1}{\sigma_{\ell}} \frac{\partial \sigma_{\ell}}{\partial \beta_{m}}
$$

Thus

$$
B_{21}=\left(\frac{\partial \log \sigma_{i}}{\partial \beta_{m}}\right)_{1 \leq i \leq n, 1 \leq m \leq n-1}
$$

and similarly

$$
B_{22}=\left(\frac{\partial \log \sigma_{i}}{\partial \alpha_{m}}\right)_{1 \leq i \leq n, 1 \leq m \leq n}
$$

Also

$$
\Gamma_{n} B_{21}=\left(\begin{array}{ccc}
\frac{\partial \log \sigma_{2}^{2} / \sigma_{1}^{2}}{\partial \beta_{1}} & \cdots & \frac{\partial \log \sigma_{2}^{2} / \sigma_{1}^{2}}{\partial \beta_{n-1}} \\
\vdots & & \vdots \\
\frac{\partial \log \sigma_{n}^{2} / \sigma_{n-1}^{2}}{\partial \beta_{1}} & \cdots & \frac{\partial \log \sigma_{n}^{2} / \sigma_{n-1}^{2}}{\partial \beta_{n-1}}
\end{array}\right)
$$


and

$$
\Gamma_{n} B_{22}=\left(\begin{array}{ccc}
\frac{\partial \log \sigma_{2}^{2} / \sigma_{1}^{2}}{\partial \alpha_{1}} & \ldots & \frac{\partial \log \sigma_{2}^{2} / \sigma_{1}^{2}}{\partial \alpha_{n}} \\
\vdots & & \vdots \\
\frac{\partial \log \sigma_{n}^{2} / \sigma_{n-1}^{2}}{\partial \alpha_{1}} & \cdots & \frac{\partial \log \sigma_{n}^{2} / \sigma_{n-1}^{2}}{\partial \alpha_{n}}
\end{array}\right) .
$$

Recalling that for any matrix $A \Gamma|| A \|_{\infty}=\max _{i} \sum_{j}\left|A_{i j}\right|$ Twe have obtained the following theorem.

Theorem 9.23 Under the bidiagonal SVD flow (9.1), the Jacobian matrix $M(t, 0)=$ $\frac{\partial(\beta(t), \alpha(t))}{\partial(\beta, \alpha)}$, satisfies

$$
M(t, 0)=\left(\begin{array}{cc}
B_{11}+t \Gamma_{n} B_{21} & B_{12}+t \Gamma_{n} B_{22} \\
B_{21} & B_{22}
\end{array}\right)(1+o(1))
$$

where the term $o(1)$ is exponentially decreasing as $t \rightarrow \infty$, and the constant matrices $B_{21}$, $B_{22}, \Gamma_{n} B_{21}, \Gamma_{n} B_{22}$ satisfy (9.19)-(9.2Q) respectively.

Also

$$
\|M(t, 0)\|_{\infty} \leq(8 n \Leftrightarrow 4) t+O(1)
$$

as $t \rightarrow \infty$.

Proof. The point to note is that Tby (the proof of the) inequality (2.4) Tthe entries of $B_{21}$ and $B_{22}$ are bounded by 1 and the entries of $\Gamma_{n} B_{21} \Gamma \Gamma_{n} B_{22}$ are bounded by 4 .

Remark 9.25 From (9.5) and (9.6)Г

$$
\alpha_{i}(t)=\log a_{i}(t)=\log \sigma_{i}+o(1), \quad 1 \leq i \leq n,
$$

and

$$
\beta_{i}(t)=\log b_{i}(t)=t \log \left(\sigma_{i+1}^{2} / \sigma_{i}^{2}\right)+O(1), \quad 1 \leq i \leq n \Leftrightarrow 1,
$$

The content of Theorem 9.23 is that the leading asymptotics can be differentiated with respect to the initial data. This in turn suggests an alternative proof of Theorem 9.23: if (9.26) and (9.27) can be shown to hold uniformaly for all initial data in a complex neighborhood of $B$ Tthen Theorem 9.23 follows immediately from Cauchy's formula. This approach can indeed be carried outTbut we present no details. (In this connection we refer the reader to [Mos75] Twhere an analysis of the asymptotics of the classical Toda lattice (tridiagonal $\Gamma$ $F(x)=x$ ) with complex initial dataTis presented.) In Section 10 ThoweverTwe will present (the outline of) a third proof of Theorem 9.23 using iterates of the gradient of one step of the SVD algorithm Tand which does not utilize the underlying flows.

Finally we note that the proof of (9.24) shows that Tmore generally

$$
\left\|M\left(t_{2}, t_{1}\right)\right\|_{\infty} \leq(8 n \Leftrightarrow 4)\left(t_{2} \Leftrightarrow t_{1}\right)+O(1),
$$

as $t_{2} \Leftrightarrow t_{1} \rightarrow \infty$. Moreover the estimate is uniform in $t_{2}>t_{1} \geq 0$. Recalling yet again the relationship between the SVD flow and the SVD algorithm Fact 4 in the introduction is finally proven by setting $t_{1}=i$ and $t_{2}=j$ in $(9.24)^{\prime}$. 


\section{The Spectrum of the One-step Jacobian of SVD}

We consider one step of SVD taking the bidiagonal matrix $B$ to the bidiagonal matrix $B^{\prime}$. Our goal in this section is to analyze the $(2 n \Leftrightarrow 2) \times(2 n \Leftrightarrow 2)$ Jacobian $K$ of the map $B \rightarrow B^{\prime} \Gamma$ expressed in terms of $x, y$ variables $\Gamma$

$$
\begin{aligned}
K & =\frac{\partial\left(x_{1}^{\prime}, \ldots, x_{n-1}^{\prime}, y_{1}^{\prime}, \ldots, y_{n-1}^{\prime}\right)}{\partial\left(x_{1}, \ldots, x_{n-1}, y_{1}, \ldots, y_{n-1}\right)} \\
& \equiv\left(\begin{array}{ll}
\frac{\partial x^{\prime}}{\partial x} & \frac{\partial x^{\prime}}{\partial y} \\
\frac{\partial y^{\prime}}{\partial x} & \frac{\partial y^{\prime}}{\partial y}
\end{array}\right),
\end{aligned}
$$

and Tin particularTto prove Fact 2 and Fact 3 of the Introduction. Observe that $K$ is the leading $(2 n \Leftrightarrow 2) \times(2 n \Leftrightarrow 2)$ submatrix of the full Jacobian $K_{n}(t=1)$ of Section $9 ; K$ is symplectic by the results of Section 8 .

We will use the notation

$$
A_{i} \equiv \prod_{j=1}^{i} a_{j}=e^{y_{i}}, \quad 1 \leq i \leq n .
$$

As before $b_{i}=e^{x_{i}} \Gamma 1 \leq i \leq n \Leftrightarrow 1$.

Our first result is formula (10.19) below $\Gamma$ which computes $K$ to relevant orders in $b_{i}$.

The SVD algorithm can be implemented by applying a sequence of $2 \times 2$ rotations to the matrix $B$ (see [GVL83]). A straightforward induction using these rotations leads to the following formulae for the entries of $B^{\prime}$.

$$
\begin{gathered}
A_{j}^{\prime}=s_{j} / r_{j}, \quad 1 \leq j \leq n, \\
b_{j}^{\prime}=\frac{r_{j+1}}{r_{j} s_{j}} s_{j-1} b_{j} a_{j+1}, \quad 1 \leq j \leq n \Leftrightarrow 1,
\end{gathered}
$$

where $r_{j}, s_{j}$ satisfy the recurrences

$$
r_{i+1}^{2}=A_{i+1}^{2}+r_{i}^{2} b_{i+1}^{2}, \quad i \geq 0,
$$

and

$$
s_{i+1}^{2}=r_{i+1}^{4}+s_{i}^{2} b_{i+1}^{2} a_{i+2}^{2}, \quad i \geq 0,
$$

where $r_{0}=s_{0} \equiv 1$.

These recurrence relations imply

$$
r_{i}^{2}=\sum_{j=0}^{i} A_{j}^{2}\left(\prod_{k=j+1}^{i} b_{k}^{2}\right)
$$

and

$$
s_{i}^{2}=\sum_{j=0}^{i} r_{j}^{4}\left(\frac{\prod_{k=j+1}^{i} b_{k}^{2}}{A_{j+1}^{2}}\right) A_{i+1}^{2},
$$

where $\prod_{k=j+1}^{i} b_{k}^{2} \equiv 1$ if $j=i$. Observe that

$$
r_{i}^{2}=r_{i}^{2}\left(A_{1}, \ldots, A_{i}, b_{1}, \ldots, b_{i}\right)
$$




$$
s_{i}^{2}=s_{i}^{2}\left(A_{1}, \ldots, A_{i+1}, b_{1}, \ldots, b_{i}\right),
$$

and hence from (10.2) and (10.3) $\Gamma$

$$
\begin{aligned}
x_{i}^{\prime} & =x_{i}^{\prime}\left(x_{1}, \ldots, x_{i+1}, y_{1}, \ldots, y_{i+1}\right) \\
y_{i}^{\prime} & =y_{i}^{\prime}\left(x_{1}, \ldots, x_{i}, y_{1}, \ldots, y_{i+1}\right) .
\end{aligned}
$$

Expansion to third order in $b^{2}=\sum_{i=1}^{n-1} b_{i}^{2}$ Tyields

$$
\begin{aligned}
& r_{i}^{2}=A_{i}^{2}+A_{i-1}^{2} b_{i}^{2}+A_{i-2}^{2} b_{i-1}^{2} b_{i}^{2}+O\left(b^{6}\right), \\
& s_{i}^{2}= A_{i}^{4}+\left(2 A_{i}^{2} A_{i-1}^{2}+A_{i-1}^{4} A_{i+1}^{2} A_{i}^{-2}\right) b_{i}^{2}+\left(2 A_{i}^{2} A_{i-2}^{2}\right. \\
&\left.+2 A_{i-1}^{2} A_{i-2}^{2} A_{i+1}^{2} A_{i}^{-2}+A_{i-2}^{4} A_{i+1}^{2} A_{i-1}^{-2}\right) b_{i-1}^{2} b_{i}^{2}+A_{i-1}^{4} b_{i}^{4}+O\left(b^{6}\right), \\
& r_{i}^{2} s_{i}^{2}= A_{i}^{6}+\left(3 A_{i}^{4} A_{i-1}^{2}+A_{i-1}^{4} A_{i+1}^{2}\right) b_{i}^{2}+\left(3 A_{i}^{4} A_{i-2}^{2}+2 A_{i-1}^{2} A_{i-2}^{2} A_{i+1}^{2}\right. \\
&\left.+A_{i}^{2} A_{i-2}^{4} A_{i+1}^{2} A_{i-1}^{-2}\right) b_{i-1}^{2} b_{i}^{2}+\left(3 A_{i}^{2} A_{i-1}^{4}+A_{i-1}^{6} A_{i+1}^{2} A_{i-1}^{-2}\right) b_{i}^{4}+O\left(b^{6}\right), \\
& r_{i+1}^{2} s_{i-1}^{2}= A_{i+1}^{2} A_{i-1}^{4}+\left(2 A_{i-1}^{2} A_{i-2}^{2} A_{i+1}^{2}+A_{i-2}^{4} A_{i}^{2} A_{i+1}^{2} A_{i-1}^{-2}\right) b_{i-1}^{2} \\
&+\left(A_{i}^{2} A_{i-1}^{4}\right) b_{i+1}^{2}+\left(A_{i+2}^{2} A_{i-2}^{4}\right) b_{i-1}^{4}+\left(2 A_{i-1}^{2} A_{i-3}^{2} A_{i+1}^{2}+2 A_{i-2}^{2} A_{i-3}^{2} A_{i}^{2} A_{i+1}^{2} A_{i-1}^{-2}\right. \\
&\left.+A_{i-3}^{4} A_{i}^{2} A_{i+1}^{2} A_{i-2}^{-2}\right) b_{i-1}^{2} b_{i-2}^{2}+\left(2 A_{i-1}^{2} A_{i-2}^{2} A_{i}^{2}\right. \\
&\left.+A_{i-2}^{4} A_{i}^{4} A_{i-1}^{-2}\right) b_{i-1}^{2} b_{i+1}^{2}+A_{i-1}^{6} b_{i}^{2} b_{i+1}^{2}+O\left(b^{6}\right) .
\end{aligned}
$$

We first compute $\partial x^{\prime} / \partial x$ Twhich is lower Hessenberg by (10.10). From (10.3)

$$
\Leftrightarrow \frac{\partial x_{i}^{\prime}}{\partial x_{j}}=\delta_{i j}+\frac{1}{2} \frac{\frac{\partial}{\partial x_{j}} r_{i+1}^{2} s_{i-1}^{2}}{r_{i+1}^{2} s_{i-1}^{2}} \Leftrightarrow \frac{1}{2} \frac{\frac{\partial}{\partial x_{j}}\left(r_{i}^{2} s_{i}^{2}\right)}{r_{i}^{2} s_{i}^{2}} .
$$

Using (10.15) and (10.16) Tand the fact that

$$
\frac{\partial}{\partial x_{i}}(\text { anything })=(\text { something }) b_{i}^{2}
$$

we obtain

$$
\left.\begin{array}{cccc}
\Leftrightarrow b_{i}^{2}\left(\frac{3}{\sigma_{1}^{2}}\right. & \left(\frac{\partial x^{\prime}}{\partial x} \Leftrightarrow I\right)= & \\
\left.+\frac{\sigma_{2}^{2}}{\sigma_{1}^{4}}+O\left(b^{2}\right)\right) & b_{2}^{2}\left(\frac{1}{\sigma_{2}^{2}}\right. & 0 & \\
b_{1}^{2}\left(\frac{2}{\sigma_{1}^{2}}+\frac{a_{2}^{2}}{a_{1}^{4}}+O\left(b^{2}\right)\right) & \Leftrightarrow b_{2}^{2}\left(\frac{3}{\sigma_{2}^{2}}+\frac{\sigma_{3}^{2}}{\sigma_{2}^{4}}+O\left(b^{2}\right)\right) & b_{3}^{2}\left(\frac{1}{\sigma_{3}^{2}}+O\left(b^{2}\right)\right) \\
\left.+\frac{a_{2}^{2}}{a_{1}^{4}}+O\left(b^{2}\right)\right) & \left.+\frac{\sigma_{3}^{2}}{\sigma_{2}^{4}}+O\left(b^{2}\right)\right) & & \\
O\left(b_{1}^{2} b^{2}\right) & b_{2}^{2}\left(\frac{2}{\sigma_{2}^{2}}+\frac{\sigma_{3}^{2}}{\sigma_{2}^{4}}+O\left(b^{2}\right)\right) & & \ddots \\
\left.+\frac{\sigma_{3}^{2}}{\sigma_{2}^{4}}+O\left(b^{2}\right)\right) & \vdots & \ddots & 0 \\
& O\left(b_{2}^{2} b^{2}\right) \ldots & b_{n-2}^{2}\left(\frac{2}{\sigma_{n-2}^{2}}+\frac{\sigma_{n-1}^{2}}{\sigma_{n-2}^{4}}+O\left(b^{2}\right)\right) & \Leftrightarrow b_{n-1}^{2}\left(\frac{3}{\sigma_{n-1}^{2}}+\frac{\sigma_{n}^{2}}{\sigma_{n-1}^{4}}+O\left(b^{2}\right)\right)
\end{array}\right)
$$


Similar formulae can be obtained for $\frac{\partial x^{\prime}}{\partial y} \Gamma \frac{\partial y^{\prime}}{\partial x}$ and $\frac{\partial y^{\prime}}{\partial y}$. In particular $\frac{\partial y^{\prime}}{\partial x}$ is lower triangular and

$$
\frac{\partial y^{\prime}}{\partial x}=\left(\begin{array}{cccc}
p_{1}^{2} b_{1}^{2} & 0 & \ldots & 0 \\
O\left(b_{1}^{2} b_{2}^{2}\right) & p_{2}^{2} b_{2}^{2} & & \\
\vdots & \vdots & \ddots & \\
O\left(b_{1}^{2} b_{n-1}^{2}\right) & O\left(b_{2}^{2} b_{n-1}^{2}\right) & \ldots & p_{n-1}^{2} b_{n-1}^{2}
\end{array}\right)
$$

where

$$
p_{i}=\sqrt{\frac{1}{\sigma_{i}^{2}}+\frac{\sigma_{i+1}^{2}}{\sigma_{i}^{4}}+O\left(b^{2}\right)}, \quad 1 \leq i \leq n \Leftrightarrow 1 .
$$

The final formulae can be written in the form

$$
K=I+\left(\begin{array}{cc}
E_{1} B^{2} & E_{2}+O_{0}\left(b^{2}\right) \\
B\left(1+B E_{3} B\right) B & B^{2} E_{4}
\end{array}\right)
$$

where

$$
\begin{aligned}
& B=\operatorname{diag}\left(p_{1} b_{1}, \ldots, p_{n-1} b_{n-1}\right), \\
& E_{2}=\left(\begin{array}{rrrr}
\Leftrightarrow 4 & 2 & & \\
2 & \Leftrightarrow 4 & \ddots & \\
& \ddots & \ddots & \\
& & & 2 \\
\bigcirc & & & \Leftrightarrow 4
\end{array}\right), \quad O_{0}\left(b^{2}\right) \text { is lower Hessenberg } \Gamma \\
& E_{1}, E_{4} \text { are lower Hessenberg with entries } \\
& \left(E_{1}\right)_{i, i+1},\left(E_{4}\right)_{i, i+1} \text { of the form } \\
& \text { (positive constant }+O\left(b^{2}\right) \text { ), }
\end{aligned}
$$

and

$$
E_{3} \text { is strictly lower triangular. }
$$

Note first that (10.20) immediately proves Fact 3 of the Introduction $\Gamma$

$$
K \rightarrow K_{\infty}=\left(\begin{array}{cc}
1 & E_{2} \\
0 & 1
\end{array}\right)
$$

as $t=k \rightarrow \infty$. Formula (10.20) can also be used to give an alternative proof of the asymptotics of $K_{n}(t)$ Tas mentioned in Remark 9.25 . Let $K(j)$ be the leading $(2 n \Leftrightarrow 2) \times$ $(2 n \Leftrightarrow 2)$ submatrix of $K_{n}(t)$ evaluated at time $t=j$. We will show that

$$
K(j)=\left(\begin{array}{cc}
A_{11}^{\prime}+j E_{2} A_{12}^{\prime} & A_{12}^{\prime}+j E_{2} A_{22}^{\prime} \\
A_{21}^{\prime} & A_{22}^{\prime}
\end{array}\right)(1+o(1)),
$$

and leave the (rather lengthy) remaining details to the reader. From (10.20) and (9.6) we have for $k$ large

$$
K(k+1, k)=K_{\infty}+\epsilon_{k},
$$


where

$$
\left\|\epsilon_{j}\right\| \leq c \rho^{j}, \quad 0<\rho<1, \quad c \text { constant. }
$$

Writing

$$
\begin{aligned}
K(j) & =\prod_{k=0}^{j-1} K(k+1, k) \\
& =K_{\infty}^{j} \prod_{k=0}^{j-1}\left(1+K_{\infty}^{-(k+1)} \epsilon_{k} K_{\infty}^{k}\right) .
\end{aligned}
$$

But

$$
\left\|K_{\infty}^{-(k+1)} \epsilon_{k} K_{\infty}^{k}\right\| \leq c^{\prime} k \rho^{k}
$$

for large $k$. Thus

$$
\left(\begin{array}{ll}
A_{11}^{\prime} & A_{12}^{\prime} \\
A_{21}^{\prime} & A_{22}^{\prime}
\end{array}\right) \equiv \lim _{j \rightarrow \infty} \prod_{k=0}^{j-1}\left(1+K_{\infty}^{-(k+1)} \epsilon_{k} K_{\infty}^{k}\right)
$$

exists and the convergence is exponential. Formula (10.25) now follows.

Our next result towards the proof of Fact 2 shows that the eigenvalues $\lambda_{j} \Gamma 1 \leq j \leq 2 n \Leftrightarrow 2 \Gamma$ of $K=K(t+1, t)$ eventually lie in Gershgorin-type disks contained in a fixed wedge with vertex $\lambda=1$ Tsymmetric about $1+i \mathrm{R}$ Tand with aperture $2 \theta$ less than $\pi$.

Figure 1:

In particular $(\operatorname{spec} K(t+1, t)) \cap \mathbf{R}$ is eventually empty.

Observe first that $K \Leftrightarrow I=K(t+1, t) \Leftrightarrow I$ can be rewritten as

$$
K \Leftrightarrow I=\left(\begin{array}{cc}
1 & 0 \\
0 & B
\end{array}\right)\left(\begin{array}{cc}
E_{1} B & E_{2}+O_{0}\left(b^{2}\right) \\
1+B E_{3} B & B E_{4}
\end{array}\right)\left(\begin{array}{cc}
B & 0 \\
0 & 1
\end{array}\right)
$$


so that the eigenvalue problem

$$
(K \Leftrightarrow I)\left(\begin{array}{l}
f^{\prime} \\
g^{\prime}
\end{array}\right)=\nu\left(\begin{array}{l}
f^{\prime} \\
g^{\prime}
\end{array}\right), \quad \lambda=1+\nu,
$$

reduces to the system

$$
\begin{aligned}
\left(E_{2}+O\left(b^{2}\right)\right) g & =\left(\nu B^{-1} \Leftrightarrow E_{1} B\right) f \\
\left(1+B E_{2} B\right) f & =\left(\nu B^{-1} \Leftrightarrow B E_{4}\right) g
\end{aligned}
$$

where

$$
\nu=\lambda \Leftrightarrow 1 \quad \text { and } \quad\left(\begin{array}{l}
f \\
g
\end{array}\right)=\left(\begin{array}{c}
B f^{\prime} \\
g^{\prime}
\end{array}\right) \neq 0 .
$$

For $t \rightarrow \infty \Gamma K(t+1, t) \rightarrow\left(\begin{array}{ll}1 & E_{2} \\ 0 & 1\end{array}\right)$ Гand so

$$
\nu \rightarrow 0
$$

As $E_{3}$ is strictly lower triangular $\Gamma\left(1+B E_{3} B\right)^{-1}$ exists (even if the $b_{j}$ are not small) and so $g \neq 0$. We normalize

$$
\|g\|^{2}=\sum_{j=1}^{n-1}\left|g_{j}\right|^{2}=1 \text {. }
$$

We have

$$
\begin{aligned}
\left(E_{2}+O_{0}\left(b^{2}\right)\right) g= & \left(\nu B^{-1}\right)\left(1+B E_{3} B\right)^{-1}(\nu B)^{-1} g+E_{1} B\left(1+B E_{3} B\right)^{-1}\left(B E_{4}\right) g \\
& \Leftrightarrow E_{1} B\left(1+B E_{3} B\right)^{-1}\left(\nu B^{-1}\right) g \Leftrightarrow\left(\nu B^{-1}\right)\left(1+B E_{3} B\right)^{-1}\left(B E_{4}\right) g \\
= & I+I I+I I I+I V .
\end{aligned}
$$

Now

$$
\begin{aligned}
I & =\left(\nu B^{-1}\right)^{2}+\nu^{2} \sum_{k=1}^{n-1}(\Leftrightarrow 1)^{k-1}\left(B^{-1}\left(B E_{3} B\right)^{k} B^{-1}\right) g \\
& =\left(\nu B^{-1}\right)^{2}+O_{1}\left(\nu^{2}\right)
\end{aligned}
$$

where $O_{1}\left(\nu^{2}\right)$ is strictly lower triangular $\Gamma$

$$
I I=O_{2}\left(b^{4}\right)+E_{1} B^{2} E_{4},
$$

where we note that

$$
\begin{gathered}
E_{1} B^{2} E_{4}=O_{2}^{\prime}\left(b^{2}\right)+\left(\begin{array}{ccccc}
0 & 0 & O\left(b_{2}^{2}\right) & 0 & \ldots \\
0 & 0 & 0 & O\left(b_{3}^{2}\right) & 0 \\
& & \ddots & \\
& & & \ldots
\end{array}\right), O_{2}^{\prime}(b) \text { is lower Hessenberg } \Gamma \\
I I I=O_{3}(\nu)
\end{gathered}
$$

and

$$
I V=O_{4}(\nu),
$$

where $O_{3}(\nu) \Gamma O_{4}(\nu)$ are again lower Hessenberg. 
Thus the eigenvalue problem becomes

$$
\left(E_{2} \Leftrightarrow\left(\nu B^{-1}\right)^{2}\right) g=\left(O_{1}\left(\mu^{2}\right)+O_{2}\left(b^{4}\right) \Leftrightarrow O_{0}\left(b^{2}\right)+E_{1} B^{2} E_{4}+O_{3}(\nu)+O_{4}(\nu)\right) g .
$$

For our present purposes all we need is that

$$
\left(E_{2} \Leftrightarrow\left(\nu B^{-1}\right)^{2}\right) g=o_{5}(1), \quad\|g\|=1
$$

which implies

$$
\begin{aligned}
\min _{j}\left|4+\left(\nu / p_{j} b_{j}\right)^{2}\right| & \leq\left(\sum_{j=1}^{n-1}\left|\Leftrightarrow 4 \Leftrightarrow\left(\nu / p_{j} b_{j}\right)^{2}\right|^{2}\left|g_{j}\right|^{2}\right)^{1 / 2} \\
& \leq\|Y\|_{2}+o_{5}(1)
\end{aligned}
$$

where $Y$ is the $(n \Leftrightarrow 1) \times(n \Leftrightarrow 1)$ matrix

$$
Y=\left(\begin{array}{cccc}
0 & 2 & & 0 \\
2 & & & \\
& \ddots & \ddots & \\
& & & 2 \\
0 & & 2 & 0
\end{array}\right), \quad\|Y\|_{2}=4 \cos \frac{\pi}{n}<4 .
$$

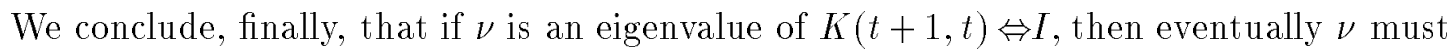
lie in one of the $2(n \Leftrightarrow 2)$ Gershgorin-type disks $D_{j}^{ \pm} \Gamma 1 \leq j \leq n \Leftrightarrow 1 \Gamma$

$$
\left\{z:\left|4+\left(z / p_{j} b_{j}\right)^{2}\right|<\rho_{n}\right\},
$$

where

$$
\|Y\|_{2}<\rho_{n} \equiv \frac{1}{2}\left(\|Y\|_{2}+4\right)<4
$$

In particularTthis establishes Fig. 1 with $\sin 2 \theta=\rho_{n} / 4$.

Inserting a free parameter $s \Gamma 0 \leq s \leq 1$ in $(10.26)$ Tas follows $\Gamma$

$$
K_{s} \Leftrightarrow I \equiv\left(\begin{array}{cc}
1 & 0 \\
0 & B
\end{array}\right)\left(\begin{array}{cc}
s E_{1} B & \Leftrightarrow 4 I+s\left(Y+O_{0}\left(b^{2}\right)\right) \\
1+s B E_{2} B & s B E_{4}
\end{array}\right)\left(\begin{array}{cc}
B & 0 \\
0 & 1
\end{array}\right),
$$

simple bookkeeping shows that we are led to an eigenvalue problem with spectrum lying in the same disks (10.37) ${ }_{j}$ Tuniformly for $0 \leq s \leq 1$ as $t \rightarrow \infty$. But the centers of the disks are the eigenvalues of $K_{0} \Leftrightarrow I=\left.K_{s}\right|_{s=0} \Leftrightarrow I$ Tand a standard continuity argument in $s$ now shows that each disk $D_{j}^{ \pm}$contains one eigenvalue of $K \Leftrightarrow I$. (If two disks overlap this means that their union contains two eigenvalues of $K \Leftrightarrow I$ Tetc.)

Recall from (9.6) that

$$
b_{i}(t) \sim b_{i}^{\infty}\left(\frac{\sigma_{i+1}}{\sigma_{i}}\right)^{2 t}, \quad 1 \leq i \leq n \Leftrightarrow 1, \quad b_{i}^{\infty}>0 .
$$

In the case that the numbers $\sigma_{i+1} / \sigma_{i} \Gamma 1 \leq i \leq n \Leftrightarrow 1$ are distinct we obtain immediately a proof of Fact 2. For in this case the disks $D_{j}^{ \pm}$are eventually disjoint $\Gamma$ and each of the 
translated disks $1+D_{j}^{ \pm}$contains precisely one of the $2(n \Leftrightarrow 2)$ eigenvalues $\{\lambda\}$ of $K$. Thus the eigenvalues are simple. Suppose $\lambda \in 1+D_{j}^{ \pm}$for some $j$. Then $\bar{\lambda}^{-1}$ is also an eigenvalue by Fact $1 \Gamma$ and must lie in the same $\operatorname{disk} \Gamma$ by a simple computation using $(10.37)_{j}$. By simplicity we must have $\lambda=\bar{\lambda}^{-1}$ Ti.e. the roots lie on the unit circle.

Remark 10.39. The idea for the above proof of Fact 2 was suggested to the authors by Gene Wayne (cf. [d1LW89]).

The remainder of this section is devoted to proving Fact 1 in the (nongeneric) case where the numbers $\sigma_{i+1} / \sigma_{i}$ are not distinct.

We show first that the eigenvalues $1+\nu$ are eventually geometrically simple. From $(10.34) \Gamma(10.33)_{I^{-}}(10.33)_{I V}$ Twe see that the eigenvalue equation has the form

$$
\left(\begin{array}{cccccc}
\Leftrightarrow 4 \Leftrightarrow\left(\frac{\nu}{p_{1} b_{1}}\right)^{2}+o(1) & 2+o(1) & O\left(b_{2}^{2}\right) & 0 & & \ldots \\
* & \Leftrightarrow 4 \Leftrightarrow\left(\frac{\nu}{p_{2} b_{2}}\right)^{2}+o(1) & 2+o(1) & O\left(b_{3}^{2}\right) & 0 & \ldots \\
* & * & \Leftrightarrow 4 \Leftrightarrow\left(\frac{\nu}{p_{3} b_{3}}\right)^{2}+o(1) & 2+o(1) & O\left(b_{4}^{2}\right) & 0 \ldots \\
\vdots & \vdots & \vdots & \ddots & \ddots & \vdots
\end{array}\right)\left(\begin{array}{l}
g_{1} \\
\cdot \\
\cdot \\
g_{n-1}
\end{array}\right)=0
$$

where the terms $*$ are bounded as $t \rightarrow \infty$. Now if $\nu$ were geometrically double $\Gamma$ we could take $g_{1}=0$. But by induction

$$
\operatorname{det}\left(\begin{array}{ccccc}
2+o(1) & O\left(b_{2}^{2}\right) & 0 & \cdots & \\
\Leftrightarrow 4 \Leftrightarrow\left(\nu / b_{2} p_{2}\right)^{2}+o(1) & 2+o(1) & O\left(b_{3}^{2}\right) & 0 & \cdots \\
& \ddots & \ddots &
\end{array}\right)=2^{n-2}+o(1) \neq 0,
$$

which implies $\left(g_{1}, \ldots, g_{n-1}\right)^{T}=0$ C contradicting $g \neq 0$. Thus the geometric multiplicity is one.

Remark 10.41. One can show that the eigenvalue equation always takes the form $(10.40) \Gamma$ even when the $b_{i}$ 's are not small $\Gamma$ provided we replace $O\left(b_{2}^{2}\right), O\left(b_{3}^{2}\right), \ldots$ by certain positive quantities. This implies $\Gamma$ in particularTthat the geometric multiplicity of any eigenvalue of $K(t, t+1)$ is at most $2 \Gamma$ for all $t$.

Next we show that for the eigenvalue problem (10.27) Tas $t \rightarrow \infty \Gamma$

$$
\left(\left(\begin{array}{c}
\bar{f}^{\prime} \\
\bar{g}^{\prime}
\end{array}\right), i J\left(\begin{array}{l}
f^{\prime} \\
g^{\prime}
\end{array}\right)\right)>0 \quad \text { if } \operatorname{Im} \lambda>0,
$$

and

$$
\left(\left(\begin{array}{c}
\bar{f}^{\prime} \\
\bar{g}^{\prime}
\end{array}\right), i J\left(\begin{array}{l}
f^{\prime} \\
g^{\prime}
\end{array}\right)\right)<0 \quad \text { if } \operatorname{Im} \lambda<0,
$$

where $(\cdot, \cdot)=(\cdot, \cdot)_{m}$ denotes the real Euclidean inner product in $\mathbf{R}^{m}$. Indeed $\Gamma$ from $(10.27) \Gamma$

$$
\begin{aligned}
\left(E_{2}+O_{0}\left(b^{2}\right)\right) g^{\prime} & =\left(\nu \Leftrightarrow E_{1} B^{2}\right) f^{\prime} \\
\left(1+B^{2} E_{3}\right) B^{2} f^{\prime} & =\left(\nu \Leftrightarrow B^{2} E_{4}\right) g^{\prime}
\end{aligned}
$$


which implies

$$
\begin{aligned}
f^{\prime} & =B^{-2}\left(1+B^{2} E_{3}\right)^{-1}\left(\nu \Leftrightarrow B^{2} E_{4}\right) g^{\prime} \\
& =\left(\nu B^{-2}\right) g^{\prime}+O(1)
\end{aligned}
$$

where we have normalized $\left\|g^{\prime}\right\|=1$. Thus

$$
\left(\bar{g}^{\prime}, f^{\prime}\right)=\nu\left\|B^{-1} g^{\prime}\right\|^{2}+O(1) .
$$

Now suppose $\left|\nu\left\|B^{-1} g^{\prime}\right\|^{2}\right|$ remains bounded as $t \rightarrow \infty$. Then $\left(\bar{g}^{\prime}, f^{\prime}\right)$ remains bounded by (10.44) and as $t \rightarrow \infty$

$$
\left(\bar{g}^{\prime},\left(E_{2} g^{\prime}\right)\right)=O\left(b^{2}\right)+\nu\left(\bar{g}^{\prime}, f^{\prime}\right) \Leftrightarrow\left(\bar{g}^{\prime}, E_{1} B^{2} f^{\prime}\right) \rightarrow 0
$$

as $b \rightarrow 0 \Gamma \nu \rightarrow 0$ and $B^{2} f^{\prime}=\left(1+B^{2} E_{3}\right)^{-1}\left(\nu \Leftrightarrow B^{2} E_{4}\right) g^{\prime} \rightarrow 0$. But this contradicts $\left|\left(\bar{g}^{\prime}, E_{2} g^{\prime}\right)\right| \geq \inf \operatorname{spec}\left(\Leftrightarrow E_{2}\right)>0$. Hence $\nu\left\|B^{-1} g^{\prime}\right\|^{2} \rightarrow \infty$ as $t \rightarrow \infty$. Formulae (10.42) and (10.43) now follow from (10.44) and Figure 1.

Suppose $\lambda \neq \bar{\lambda}^{-1}$ is an eigenvalue of $K \Gamma K\left(\begin{array}{l}f^{\prime} \\ g^{\prime}\end{array}\right)=\lambda\left(\begin{array}{l}f^{\prime} \\ g^{\prime}\end{array}\right)$. Then as $K$ is symplectic $\Gamma$

$$
\left.\left(\left(\begin{array}{c}
\bar{f}^{\prime} \\
\bar{g}^{\prime}
\end{array}\right), i J\left(\begin{array}{l}
f^{\prime} \\
g^{\prime}
\end{array}\right)\right)=\overline{\left(K\left(\begin{array}{l}
f^{\prime} \\
g^{\prime}
\end{array}\right)\right.}, i J K\left(\begin{array}{l}
f^{\prime} \\
g^{\prime}
\end{array}\right)\right)=\bar{\lambda} \lambda\left(\left(\begin{array}{l}
\bar{f}^{\prime} \\
g^{\prime}
\end{array}\right), i J\left(\begin{array}{l}
f^{\prime} \\
g^{\prime}
\end{array}\right)\right)
$$

which implies $\left(\left(\frac{f^{\prime}}{\bar{g}^{\prime}}\right), i J\left(\begin{array}{l}f^{\prime} \\ g^{\prime}\end{array}\right)\right)=0$ as $\bar{\lambda} \lambda \neq 1$ Ccontradicting $(10.42) \Gamma(10.43)$. (Note $\operatorname{Im} \lambda \neq 0 \Gamma$ by Figure 1). Thus $|\lambda|^{2}=\lambda \bar{\lambda}=1$. Furthermore $\Gamma$ by our previous calculations $\Gamma$ has geometric multiplicity one. Suppose $\lambda$ corresponds to a nontrivial Jordan block $\Gamma$

$$
\begin{aligned}
K=U\left(\begin{array}{cccc}
\lambda & 1 & & \\
0 & \lambda & & \\
& 0 & & \\
\vdots & & \ddots & \ddots \\
\vdots & &
\end{array}\right) U^{-1}, \\
K^{T}=U^{-T}\left(\begin{array}{ccc}
\lambda & 0 & \cdots \\
1 & \lambda & 0 \\
& \ddots & \ddots
\end{array}\right) U^{T} .
\end{aligned}
$$

for some invertible matrix $U$. Set $\left(\begin{array}{l}f^{\prime} \\ g^{\prime}\end{array}\right)=U e_{1}$; then $K\left(\begin{array}{l}f^{\prime} \\ g^{\prime}\end{array}\right)=\lambda\left(\begin{array}{l}f^{\prime} \\ g^{\prime}\end{array}\right)$. But $K^{T} J\left(\frac{\bar{f}^{\prime}}{g^{\prime}}\right)=$ $J K^{-1}\left(\bar{f}^{\prime}\right)=\bar{\lambda}^{-1} J\left(\bar{f}^{\prime}\right)$ Tso that $J \bar{f}=\sum_{j \neq 1} c_{j} U^{-T} e_{j}$ for suitable constants $c_{j}$ Twhere $j=1$ is excluded as $U^{-T} e_{1}$ is not an eigenvector of $K^{T}$. Thus

$$
\left(\left(\begin{array}{c}
\bar{f}^{\prime} \\
\bar{g}^{\prime}
\end{array}\right), i J\left(\begin{array}{c}
f^{\prime} \\
g^{\prime}
\end{array}\right)\right)=\Leftrightarrow\left(\left(\begin{array}{c}
f^{\prime} \\
g^{\prime}
\end{array}\right), i J\left(\begin{array}{c}
\bar{f}^{\prime} \\
\bar{g}^{\prime}
\end{array}\right)\right)=\Leftrightarrow \sum_{j \neq 1} c_{j}\left(U e_{1}, U^{-T} e_{j}\right)=0
$$

again contradicting $(10.42) \Gamma(10.43)$. We conclude that $\lambda$ is algebraically simple $\Gamma$ and this concludes the proof of Fact 2. 
Figure 2:

In the initial numerical experiments mentioned in the Introduction $\Gamma$ in addition to Facts 1, 2 and 3 Tit was observed that whenever an eigenvalue $\lambda$ came off the unit circle for finite $t=j$ The break occurred through $\lambda=\Leftrightarrow 1 \Gamma$

The preceding computations give some insight into $\Gamma$ but $\Gamma$ as yet $\Gamma$ not a complete proof of $\Gamma$ this phenomenon. Indeed $\Gamma i f \lambda_{1} \neq \lambda_{2}$ are two distinct eigenvalues of $K \Gamma K h_{i}=\lambda_{i} h_{i} \Gamma i=1,2 \Gamma$ with positive imaginary parts $\Gamma$ then arguing as above $\Gamma\left(\bar{h}_{1}, i J h_{2}\right)=0$. But then for $t$ large when the spectrum of $K(t+1, t)$ is simpleTwe must have

$$
(\bar{h}, i J h)>0
$$

for all nonzero $h$ in $V_{+}(t) \equiv \operatorname{span}\{w: K(t+1, t) w=\lambda w, \operatorname{Im} \lambda>0\}$. But $V_{+}(t)$ is clearly continuous in $t$ as long as the spectrum of $K(t+1, t)$ does not cross the real axis. Let $t_{0}<\infty$ be the last time for which spec $K(t+1, t) \cap \mathbf{R} \neq \emptyset$. (Such a time mayTof course exist.) It follows by continuity that (10.45) holds for all $t>t_{0}$ Tand using arguments similar to those aboveTone obtains the following result: viewed backwards in time from $t=\infty \Gamma$ the matrix $K(t+1, t)$ is diagonalizable with spec $K(t+1, t) \subset\{\lambda:|\lambda|=1\}$ Гuntil such a time $t_{0}$ that an eigenvalue touches the real axis at $\lambda=+1$ or $\lambda=\Leftrightarrow 1$.

At this point Thowever Tit is not clear how to rule out the case $\lambda=1$.

Remark 10.46 The reader familiar with dynamical stability theory will recognize that the above computations are modeled on the strong stability theory of Krein ([Kre50Г Kre55]; see also [GL55] [ $[$ Mos58]) for symplectic matrices. 


\section{Numerical Experiments}

In this section we summarize numerical experiments which support the error analysis of sections 5 and 6 . The first set of experiments determines the average number of QR steps necessary for convergence $a$ and were performed in [DK88]; we just cite the needed data here. The second set describes the growth of $\|M(t)\|$ for the same set of test problems. The first set of experiments were performed using Fortran on a SUN 4/260 in IEEE standard double precision floating point arithmetic [IEE85]; the machine precision $\varepsilon=2^{-53} \approx 10^{-16}$ and the range of representable numbers is approximately $10^{ \pm 308}$. The second set of experiments were performed using Matlab on the same SUN 4/260 using the same arithmetic.

The test matrices were the same 105 bidiagonal matrices in 12 classes used in [DK88]:

Class 1: These eight matrices are graded in the usual way from large at the upper left to small at the lower right. Four of the matrices are 10 by 10 and four are 20 by 20 . The singular values range from 1 to $10^{-90}$ in some examples.

Class 2: This class is identical to class 1 except the order of the entries on the diagonal and superdiagonal are reversed. Thus these matrices are graded from small at the upper left corner to large at the lower right.

Class 3: These eight 20 by 20 and 40 by 40 matrices are obtained by abutting those in class 1 with their reversals in class 2 . Thus each matrix is small at the upper left $\Gamma$ large in the middle and small again at the lower right.

Class 4: These eight 20 by 20 and 40 by 40 matrices are obtained by abutting those in class 2 with their reversals in class 1 . Thus each matrix is large at the upper left $\Gamma$ small in the middle and large again at the lower right.

Class 5: These eight matrices are obtained from class 1 by reversing the order of the superdiagonals. Thus the diagonal is graded from large at the upper left to small at the lower right Tand the superdiagonal is graded in the opposite direction.

Class 6: These eight matrices are obtained from class 5 by reversing the order of both the diagonals and superdiagonals. Thus the diagonal is graded from small at the upper left to large at the lower right Tand the superdiagonal is graded in the opposite direction.

Class 7: These sixteen matrices are all small on the diagonal and mostly large on the offdiagonal. The diagonals range from $10^{-2}$ down to $10^{-16}$ and the diagonals are mostly 1 with occasional small values.

Classes 8-11: The ten 20 by 20 matrices in each class are generated by letting each bidiagonal entry be a random number of the form $r \cdot 10^{i} \Gamma$ where $r$ is a random number uniformly distributed between $\Leftrightarrow .5$ and .5 Tand $i$ is a random integer. In class $8 \Gamma i$ is uniformly distributed from 0 to $\Leftrightarrow 15$. In class $9 \Gamma i$ is uniformly distributed from 0 to $\Leftrightarrow 10$. In class $10 \Gamma i$ is uniformly distributed from 0 to $\Leftrightarrow 5$. In class $11 \Gamma i$ is identically 0 . Thus Tin class 11 each matrix entry is simply uniformly distributed on $[\Leftrightarrow .5, .5]$. 
Class 12: This one 41 by 41 matrix is graded in as in class 1 with the ratio of adjacent entries being $10^{-.1} \approx .79$. Each offdiagonal entry is identical to the diagonal entry below it. This very dense grading leads to different convergence properties than for the matrices in class 1 which is why we put this example in a separate class.

Now we describe the results of the first experiment $\Gamma$ which computes the number of QR steps needed for convergence with relative error tolerance tol $=100 \varepsilon \approx 10^{-14}$. This is the number $m$ in the statement of Theorem 6.1. ActuallyГ we compute a related quantity which is more closely related to the actual work done: the number of "QR inner loops" divided by $n(n+1) / 2 \Gamma$ where $n$ is the matrix dimension and one "QR inner loop" is one pass through the inner loop of the $\mathrm{QR}$ algorithm (shifted or unshifted). The reason for choosing this statistic is as follows. The usual rule of thumb for the number of QR steps it takes to compute the SVD is two steps per singular value [Par80]. If convergence always takes place at the end of the matrix Tthis means there will be 2 steps on a matrix of length $i \Gamma$ for $i=n, n \Leftrightarrow 1, \ldots, 3$ (two by two matrices are handled specially). Thus 5 since one QR step on a matrix of length $i$ consists of $i$ "QR inner loops" $T$ we expect an average of about $n(n+1)$ "QR inner loops" for the entire SVD. ThusTthe quantity "QR inner loops" divided by $n(n+1) / 2$ should be a measure of the difficulty of computing the SVD of a matrix which is independent of dimension Tand we expect it to equal 2 on the average. For each of the twelve problem classes and for both the algorithm of section 3 and the standard SVD algorithm [BDMS79] Tthe minimum Table 1.

\begin{tabular}{|r|rrr|rrr|}
\hline \multicolumn{8}{|c|}{ Table 1: QR inner loops $/(n(n+1) / 2)$} \\
\hline Class & \multicolumn{3}{|c|}{ Standard SVD } & \multicolumn{3}{|c|}{ New SVD } \\
& \multicolumn{1}{|c}{ Min } & Avg & Max & Min & Avg & Max \\
\hline 1 & .60 & .90 & 1.33 & .09 & .49 & 1.11 \\
2 & .60 & 1.94 & 3.07 & .09 & .49 & 1.11 \\
3 & .61 & .85 & 1.19 & .56 & .82 & 1.19 \\
4 & .32 & 1.04 & 1.80 & .35 & .60 & 1.04 \\
5 & .07 & .45 & 1.11 & .09 & .57 & 1.42 \\
6 & .07 & .40 & .93 & .09 & .57 & 1.42 \\
7 & .10 & 1.32 & 2.31 & .10 & 1.04 & 1.85 \\
8 & .41 & .64 & .95 & .26 & .49 & .77 \\
9 & .79 & .94 & 1.29 & .57 & .75 & .93 \\
10 & 1.07 & 1.29 & 1.57 & 1.04 & 1.22 & 1.48 \\
11 & 1.97 & 2.26 & 2.52 & 2.06 & 2.20 & 2.41 \\
12 & 1.53 & 1.53 & 1.53 & 2.96 & 2.96 & 2.96 \\
\hline
\end{tabular}

Since Class 11 corresponds to matrices with uniform random entries $\Gamma$ we see that the rule of thumb of $2 \mathrm{QR}$ steps per singular value is justified. In the worst case Class 12 Tthe new SVD algorithm takes $3 \mathrm{QR}$ steps per eigenvalue. In the other classes it takes many fewer step to reach convergence. Thus Tin practice we can bound the number of QR steps by $m=3 n$ in order to obtain an upper bound depending only on the matrix dimension $n$ 
in Theorem 6.1. Of course Tafter the algorithm has been run $m$ is easily available 5 so that Theorem 6.1 could be used to get less pessimistic bounds.

The second set of experiments measured $\|M(j, 0)\|_{\infty}$ for the same test cases as above. We computed $M(j, 0)$ as follows. The first order perturbation theory in Lemmas 5.2 and 5.4 can be seen as computing the linear operator $M(i+1, i)$ which maps the relative errors $\epsilon_{a_{1}}, \ldots, \epsilon_{a_{n}}, \epsilon_{b_{1}}, \ldots, \epsilon_{b_{n-1}}$ in the entries of the bidiagonal matrix $B$ to the relative errors $\epsilon_{a_{1}^{\prime}}, \ldots, \epsilon_{a_{n}^{\prime}}, \epsilon_{b_{1}^{\prime}}, \ldots, \epsilon_{b_{n-1}^{\prime}}$ in the entries of the bidiagonal matrix $B^{\prime}$ after one zero-shift $\mathrm{QR}$ step. The entries of this matrix are computed as products of the 2 by 2 matrices appearing in the proofs of Lemmas 5.2 and $5.4 \Gamma$ and so the entries of $M(i+1, i)$ are complicated polynomials in the sines of cosines of rotation angles occuring during the running of the algorithm. We obtain $M(j, 0)=M(j, j \Leftrightarrow 1) \cdot M(1,0)$ via matrix multiplication.

The experiments were performed by taking each one of the 105 test matrices and running zero-shift QR until $M(i+1, i)$ had converged to its asymptotic value $\mathrm{Q}$ and the graph of $\|M(j, 0)\|_{\infty}$ versus $j$ had converged to a straight line; this convergence was determined by examining the graph. (This is not the same as computing all the $M(j, i)$ arising during the running of the overall hybrid algorithm on the test casesTbut is nonetheless a thorough test of our predicted upper bound $(8 n \Leftrightarrow 4)(j \Leftrightarrow i)+O(1)$ of Theorem 9.23 on $\|M(j, i)\|_{\infty}$.) For each test matrix the computed values of $\|M(j, 0)\|_{\infty}$ were analyzed as follows:

1. Let $j_{\max }$ be the number of $\mathrm{QR}$ steps taken and $n$ the matrix dimension.

2. Let $s \equiv\left(\left\|M\left(j_{\max }, 0\right)\right\|_{\infty} \Leftrightarrow\left\|M\left(j_{\max -1}, 0\right)\right\|_{\infty}\right) / n$ be the asymptotic rate of growth of $\|M(j, 0)\|_{\infty}($ divided by $n)$.

3. Let $r \equiv \max _{1 \leq j \leq j_{\max }}\left(\|M(j, 0)\|_{\infty} \Leftrightarrow n \cdot s \cdot j\right)$. Then for all $1 \leq j \leq j_{\max }$ we have $\|M(j, 0)\|_{\infty} \leq n s j+r$. In other words $\Gamma$ the line $n s j+r$ is the tightest affine upper bound to $\|M(j, 0)\|_{\infty}$.

4. Let $t=\max _{1 \leq j \leq j_{\max }}\left(n s j+r \Leftrightarrow\|M(j, 0)\|_{\infty}\right)$ be the maximum amount the straight line $n s j+r$ overestimates $\|M(j, 0)\|_{\infty}$.

\begin{tabular}{|r|rr|rrr|}
\hline \multicolumn{6}{|c|}{ Table 2: Growth Statistics for $\|M(j, 0)\|_{\infty}$} \\
\hline Class & \multicolumn{2}{|c|}{$j_{\max }$} & $\max s$ & $\max r$ & $\max t$ \\
& $\min$ & $\max$ & & & \\
\hline 1 & 5 & 20 & 2.00 & -10.00 & .26 \\
2 & 10 & 80 & 2.00 & -.02 & 1.23 \\
3 & 10 & 80 & 2.18 & -8.67 & 4.63 \\
4 & 20 & 90 & 1.86 & -7.16 & 3.34 \\
5 & 20 & 90 & 2.10 & -1.00 & 16.02 \\
6 & 20 & 90 & 2.10 & 1.00 & 18.00 \\
7 & 20 & 90 & 4.01 & -19.00 & 18.00 \\
8 & 30 & 30 & 5.06 & 24.73 & 151.28 \\
9 & 40 & 40 & 4.00 & 18.16 & 59.93 \\
10 & 40 & 40 & 3.41 & 26.29 & 49.11 \\
11 & 40 & 40 & 4.15 & 8.71 & 27.58 \\
\hline
\end{tabular}


Table 2 summarizes the values of $s \Gamma r$ and $t$ computed. Columns 2 and 3 give the minimum and maximum values of $j_{\max }$ for the given class. Columns $4 \Gamma 5$ and 6 give the maximum values of $r \Gamma s$ and $t$ Trespectively $\Gamma$ in each class.

The largest overshoot 151.28 corresponds to a $16 \%$ change in $n s j_{\max }+r \Gamma$ and small deviation from linearity. Excluding this case $\mathrm{T}$ the maximum deviation is less than $5 \%$. So even though the analysis leading to the linear growth bound on $\|M(j, 0)\|_{\infty}$ was asymptotic $\Gamma$ we find linear growth sets in quite early Tmuch earlier than we can currently explain.

The smallest values $\underline{s}$ and $\underline{r}$ which satisfy $n \underline{s} j+\underline{r} \geq n s j+r$ for all $105(s, r)$ pairs and all $j$ are $\underline{s}=5.06$ and $\underline{r}=0$. This justifies the claims made earlier and used in the error analyses of sections 5 and 6 . 


\section{Conclusions}

We have proven that the singular values of a bidiagonal matrix are much less sensitive to relative perturbations in the matrix entries than previously thought: the uncertainty of singular vector $v_{i}$ is proportional to the reciprocal of the relative gap $\min _{j \neq i}\left|\sigma_{i} \Leftrightarrow \sigma_{j}\right| /\left(\sigma_{i}+\sigma_{j}\right)$ rather than the reciprocal of the absolute gap $\min _{j \neq i}\left|\sigma_{i} \Leftrightarrow \sigma_{j}\right| / \sigma_{\max }$. When the matrix has two or more tiny singular values $\Gamma$ the relative gap can be much larger than the absolute gap $\Gamma$ so the bound is much tighter.

We have also shown that the algorithm in [DK88] is capable of computing the singular values to this higher accuracy. The proof involves a new analysis of the stopping criterion $\Gamma a s$ well as showing that rounding errors during the zero-shift QR algorithm accumulate slowly. This latter analysis is facilitated by associating to zero-shift QR a Hamiltonian differential equation which interpolates the iterates of the algorithm. In contrast to many eigenvalue algorithms where the underlying Hamiltonian structures are Lie-Poisson structures (seeTe.g.T [DLNT86 DLT89]) There the underlying structure is a so-called Sklyanin structure. The canonical variables on the appropriate symplectic leaves for the bidiagonal case turn out to be linear combinations of logarithms of the matrix entries. The differential equation shows that these canonical variables are relatively insensitive to changes in the initial conditions. Since the canonical variables are essentially logarithms of matrix entries $\Gamma$ this means that the logarithms of the matrix entries are insensitive. This in turn means that small relative errors (such as rounding errors) in the matrix entries grow slowly.

If the initial matrix is not bidiagonalTthen reduction to bidiagonal form may introduce errors so large as to swamp the finer bounds of the bidiagonal SVD. One possibility is to use Jacobi's method if the original matrix is dense [DV90]. A situation where bidiagonal reduction is sufficiently accurate is the symmetric positive definite tridiagonal eigen problem $\Gamma$ where the reduction is performed via Cholesky [BD88].

The algorithm described here will be part of the LAPACK linear algebra library for supercomputers [DDDC*87]. 


\section{Acknowledgements}

Percy Deift acknowledges the support of NSF grant DMS-8802305. James Demmel acknowledges the support of NSF grants DCR-8552474 and ASC-8715728 and DARPA grant F49620-87-C-0065. Luen-Chau Li acknowledges the support of NSF grant DMS-8704097. Carlos Tomei thanks CNPq TBrazil. The authors also thank W. KahanTwho while looking over the shoulder of the second author at a column of numbers noticed that they appeared in reciprocal pairs. They also thank Gene Wayne $\mathrm{W}$ who suggested the idea of the proof of Fact 2. Finally they acknowledge the help of Socrates Rivera who made many of the initial calculations in section 10 .

\section{References}

[Ad179] M. Adler. On a trace functional for formal pseudodifferential operators and the symplectic structure of the Korteweg-de Vries type equations. Inv. Math. 50:219-248Г1979.

[Arn78] V. I. Arnold. Mathematical Methods of Classical Mechanics. Springer-VerlagT New York 1978.

[BD88] J. Barlow and J. Demmel. Computing Accurate Eigensystems of Scaled Diagonally Dominant Matrices. Computer Science Dept. Technical Report 421T Courant InstituteT New YorkT NYT December 1988. submitted to SIAM J. Num. Anal.

[BDMS79] J. BunchГJ. DongarraГC. MolerTand G. W. Stewart. LINPACK User's Guide. SIAMГPhiladelphiaГРАГ1979.

[Chu86] M. Chu. A differential equation approach to the singular value decomposition of bidiagonal matrices. Lin. Alg. ApplГ 80:71-80Г1986.

[CL55] E. A. Coddington and N. Levinson. Theory of ordinary differential equations. McGraw-HillГNew YorkT1955.

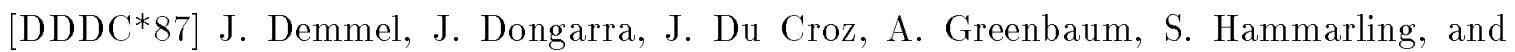
D. Sorensen. Prospectus for the Development of a Linear Algebra Library for High-Performance Computers. Mathematics and Computer Science Division Report ANL/MCS-TM-97ГArgonne National LaboratoryГArgonneГILT September 1987.

[Dem84] J. Demmel. Underflow and the reliability of numerical software. SIAM Journal on Scientific and Statistical Computing 5(4):887-919ГDec 1984.

[DK70] C. Davis and W. Kahan. The rotation of eigenvectors by a perturbation III. SIAM Journal of Numerical Analysis $\mathrm{T} 7: 248-263 \Gamma 1970$.

[DK88] J. Demmel and W. Kahan. Accurate Singular Values of Bidiagonal Matrices. SIAM J. Sci. Stat. Comp.Tv. 11Гn. 5Гpp. 873-912ГSept 1990. 
[d1LW89] R. de la Llave and C. E. Wayne. Whiskered and low dimensional tori in nearly integrable Hamiltonian systems. PreprintГ1989.

[DLNT86] P. DeiftTL. C. LiTT. NandaTand C. Tomei. The Toda flow on a generic orbit is integrable. Comm. Pure Appl. Math.Г39:183-232Г1986.

[DLT85] P. Deift L. C. LiTand C. Tomei. Toda flows with infinitely many variables. Jour. Func. Anal.Г64:358-402Г1985.

[DLT89] P. DeiftTL. C. LiTand C. Tomei. Matrix factorizations and integrable systems. Comm. Pure Appl. Math.Г42:443-521Г1989.

[DNT83] P. Deift T. NandaГand C. Tomei. Differential equations for the symmetric eigenvalue problem. SIAM J. Num. Anal.Г20:1-22Г1983.

[Dri87] K. R. Driessel. On finding the singular values and singular vectors of a matrix by means of an isosingular gradient flow. Idaho State University preprint $\Gamma$ 1987.

[DV90] J. Demmel and K. Veselić. Jacobi's Method is More Accurate than QR. to appear in SIAM J. Mat. Anal. Appl.

[GK65] G. Golub and W. Kahan. Calculating the singular values and pseudo-inverse of a matrix. SIAM Journal of Numerical Analysis (Series B)Г 2(2):205-224Г 1965 .

[GL55] I. M. Gelfand and V. B. Lidsky. On the structure of regions of stability of linear canonical systems of differential equations with periodic coefficients (transl. AMS 2(8)(1958)143-181). Usp. Math. Nauk.Г10(1):3-40Г1955.

[GVL83] G. Golub and C. Van Loan. Matrix Computations. Johns Hopkins University PressГBaltimoreГMDГ1983.

[IEE85] IEEE Standard for Binary Floating Point Arithmetic ANSI/IEEE Std 7541985. IEEEГ1985.

[Kah68] W. Kahan. Accurate Eigenvalues of a Symmetric Tridiagonal Matrix. Com-

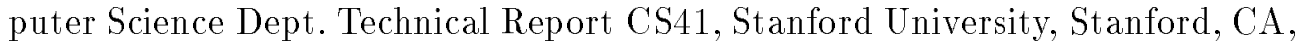
July 1966 (revised June 1968).

[Kos79] B. Kostant. The solution to a generalized Toda lattice and representation theory. Adv. in Math.Г34:195-338Г1979.

[Kre50] M. G. Krein. A generalization of several investigations of A. M. Lyapounov. Dokl. Akad. NaukГ73:445-448Г1950.

[Kre55] M. G. Krein. The basic propositions of the theory of $\lambda$-zones of stability of a canonical system of linear differential equations with periodic coefficients $\Gamma$ Pamyati A. A. Androvna. Izvestia Akad. Nauk.Г413-498Г1955. 
[KvM75] M. Kac and P. van Moerbeke. On some periodic Toda lattice. Proc. Nat. Acad. Sci., USAГ72:1627-1629Г1975.

[LPar] L. C. Li and S. Parmentier. Nonlinear Poisson structures and $r$-matrices. Comm. Math Phys.Tto appear.

[Mos58] J. Moser. New aspects in the theory of stability of Hamiltonian systems. Comm. Pure Appl. Math.Г11:81-114Г1958.

[Mos75] J. Moser. Finitely many mass points on the line under the influence of an exponential potential - an integrable system, in: Dynamical Systems Theory

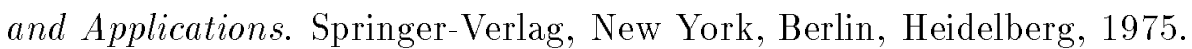

[Par80] B. Parlett. The Symmetric Eigenvalue Problem. Prentice Hall Englewood CliffsTNJT1980.

[Sem84] M. Semenov-Tyan-Shanskii. What is a classical r-matrix? Funct. Anal. Appl. $259-272 \Gamma 1984$.

[Sem85] M. Semenov-Tyan-Shanskii. Dressing transformations and Poisson group actions. Publ. RIMST21:1237-1260Г1985.

[Sym80] W. W. Symes. Hamiltonian group actions and integrable systems. Physica 1D:339-374Г1980.

[Sym82] W. W. Symes. The QR algorithm for the finite nonperiodic Toda lattice. PhysicaГ4D:275-280Г1982.

[Wat84] D. S. Watkins. Isospectral flows. SIAM Review 26:379-391Г1984.

[Wei85] A. Weinstein. The local structure of Poisson manifolds. J. Diff. Geom.T18:523$557 \Gamma 1985$.

[Wil65] J. H. Wilkinson. The Algebraic Eigenvalue Problem. Oxford University PressT Oxford $\Gamma 1965$. 